\title{
Super Yang-Mills and $\theta$-exact Seiberg-Witten map: absence of quadratic noncommutative IR divergences
}

\author{
Carmelo P. Martin, ${ }^{a}$ Josip Trampetic ${ }^{b, 1}$ and Jiangyang You ${ }^{c}$ \\ ${ }^{a}$ Departamento de Fúsica Teórica I, Facultad de Ciencias Físicas, \\ Universidad Complutense de Madrid, \\ 28040-Madrid, Spain \\ ${ }^{b}$ Max-Planck-Institut für Physik, (Werner-Heisenberg-Institut), \\ Föhringer Ring 6, D-80805 München, Germany \\ ${ }^{c}$ Divisions of Theoretical Physics, Rudjer Bošković Institute, \\ P.O.Box 180, HR-10002 Zagreb, Croatia \\ E-mail: carmelop@fis.ucm.es, trampeti@mppmu.mpg.de, \\ youjiangyang@gmail.com
}

ABSTRACT: We compute the one-loop 1PI contributions to all the propagators of the noncommutative (NC) $\mathcal{N}=1,2,4$ super Yang-Mills (SYM) U(1) theories defined by the means of the $\theta$-exact Seiberg-Witten (SW) map in the Wess-Zumino gauge. Then we extract the UV divergent contributions and the noncommutative IR divergences. We show that all the quadratic noncommutative IR divergences add up to zero in each propagator.

KeYwords: BRST Quantization, Gauge Symmetry, Non-Commutative Geometry, Supersymmetric gauge theory

ArXiv ePrint: 1602.01333

\footnotetext{
${ }^{1}$ On leave of absence from the Rudjer Bošković Institute, Zagreb, Croatia.
} 


\section{Contents}

1 Introduction 1

2 Noncommutative $\mathcal{N}=1 \mathrm{SYM} \mathrm{U}(1)$ theory and the $\theta$-exact SW map 2

2.1 The action 5

2.2 The photon one-loop contributions to the photon polarization tensor 6

$\begin{array}{lll}2.2 .1 & \text { The photon-bubble diagram } & 8\end{array}$

2.2.2 The photon-tadpole diagram 9

2.3 The photino one-loop contributions to the photon polarization tensor $\quad 10$

$\begin{array}{ll}2.3 .1 \text { The photino-tadpole diagram } & 10\end{array}$

$\begin{array}{ll}\text { 2.3.2 The photino-bubble diagram } & 11\end{array}$

2.4 The one-loop SUSY-auxiliary field contributions to the photon propagator 12

$\begin{array}{ll}2.5 & \text { The one-loop photino 1-PI two point function } \\ \end{array}$

3 Minimal action of the noncommutative adjoint scalar field $\quad \mathbf{1 4}$

$\begin{array}{ll}3.1 \text { Scalar one-loop contributions to the photon polarization tensor } & 15\end{array}$

$\begin{array}{lll}3.1 .1 & \text { The scalar-bubble diagram } & 15\end{array}$

$\begin{array}{ll}3.1 .2 & \text { The scalar-tadpole diagram } \\ & 15\end{array}$

3.2 The photon one-loop contribution to scalar 1-PI two point function $\quad 17$

4 Noncommutative $\mathcal{N}=2$ SYM U(1) theory and the $\theta$-exact SW map 18

$\begin{array}{ll}\text { 4.1 The one-loop 1PI two-point function for photon field } a_{\mu} & 19\end{array}$

$\begin{array}{ll}\text { 4.2 The one-loop 1PI two-point function for the scalar } \phi & 19\end{array}$

4.3 The one-loop 1PI two-point function for photinos $\lambda_{\alpha}$ and $\psi_{\alpha} \quad 20$

5 Noncommutative $\mathcal{N}=4$ SYM U(1) theory and the $\theta$-exact SW map 21

5.1 The one-loop 1PI two-point function for massless vector field $a_{\mu} \quad 22$

5.2 The one-loop 1PI two-point function for the scalar $\phi_{m} \quad 22$

$\begin{array}{ll}\text { 5.3 The one-loop 1PI two-point function for } \lambda_{\alpha i} & 22\end{array}$

6 Effect of gauge fixing on photon two point function $\quad 24$

6.1 The noncommutative Feynman gauge fixing action 24

6.2 One-loop contributions from the new NC gauge fixing action 25

7 Gauge fixing contribution without integrating out BRST-auxiliary field 27

$\begin{array}{lll}7.1 & \text { Formal analysis } & 28\end{array}$

7.2 The action of the gauge and BRST-auxiliary fields and Feynman rules 30

7.3 One-loop contributions to the photon effective action up to the quadratic $\begin{array}{ll}\text { order, from the BRST auxiliary field } B & 31\end{array}$

8 Summary and discussion $\quad 34$ 


\section{Introduction}

The one-loop UV/IR mixing structure of noncommutative $\mathcal{N}=1$ super Yang-Mills theory defined in terms of the noncommutative fields was studied some years ago in a number of papers [1-5]. The outcome was the famous quadratic noncommutative IR divergences which occur in the one-loop gauge field propagator of the non-supersymmetric version of the theory cancel here due to Supersymmetry. The one-loop gauge field propagator still carries a logarithmic UV divergence -a simple pole in Dimensional Regularization- and the dual logarithmic noncommutative IR divergence $\ln \left(p^{2}(\theta p)^{2}\right)$ as a result of the UV/IR mixing being at work. By increasing the number of supersymmetries of the noncommutative Yang-Mills theory one makes the UV behaviour of the theory softer and eventually finite for $\mathcal{N}=4$ [6], at which point noncommutative IR divergences cease to exist $[7,8]$. In the $\mathcal{N}=2$ super Yang-Mills case, there still remain logarithmic UV divergences at one-loop in the twopoint function which give rise via UV/IR mixing to the corresponding IR divergences $[1,9]$. That noncommutative $\mathcal{N}=4$ super Yang-Mills has a smooth commutative limit has been shown in ref. [10].

It is known that classically noncommutative gauge field theories admit a dual formulation in terms of ordinary fields, a formulation that is obtained by using the celebrated Seiberg-Witten map [11]. However we still do not know whether this duality holds at the quantum level, i.e., whether the quantum theory defined in terms of noncommutative fields is the same as the ordinary quantum theory — called the dual ordinary theory - whose classical action is obtained from the noncommutative action by using the Seiberg-Witten map. The existence of UV/IR mixing effects in noncommutative field theory defined in terms of noncommutative fields is thought to be a characteristic feature of those field theories. It is thus sensible to think such effects should also occur in the ordinary dual theory obtained, as previously explained, by using the Seiberg-Witten map. That these UV/IR mixing effects actually occur in the propagator of the gauge field of the dual ordinary theory was first shown in ref. [12] by using the $\theta$-exact Seiberg-Witten map expansion $[13,14]$. The 
analysis of the properties and phenomenological implications of the UV/IR mixing effects that occur in noncommutative gauge theories defined by means of the $\theta$-exact SeibergWitten map has been pursued in refs. [15-18].

Up to the best of our knowledge no analysis of the UV and the noncommutative IR structures of noncommutative super Yang-Mills theory defined by means of the $\theta$-exact Seiberg-Witten map has been carried out as yet. In particular, it is not known whether the cancellation of the quadratic noncommutative IR divergences of the gauge-field propagator that occurs, as we mentioned above, in noncommutative super Yang-Mills theory defined in terms of noncommutative fields also works in its dual ordinary theory. The answer to this question is far from obvious since Supersymmetry is linearly realized in terms of the noncommutative fields - and thus there exists a superfield formalism — but is non-linearly realized — see ref. [19] — in terms of the ordinary fields of the dual ordinary theory defined by means of the Seiberg-Witten map. It has long been known that the proper definition of theories with non-linearly realized symmetries is a highly non-trivial process.

The purpose of this paper is to work out all the one-loop 1PI two-point functions, and analyze the UV and noncommutative IR structures of those functions, in noncommutative $\mathrm{U}(1) \mathcal{N}=1,2$ and 4 super Yang-Mills theories in the Wess-Zumino gauge, when those theories are defined in terms of ordinary fields by means of the $\theta$-exact Seiberg-Witten maps. To analyze the gauge dependence of the UV and noncommutative IR of the gauge field two-point functions we shall consider to types of gauge-fixing terms for the ordinary gauge field: the standard ordinary Feynman gauge-fixing term and the noncommutative Feynman gauge-fixing term.

The layout of this paper is as follows. Section 2 is devoted to the computation of the one-loop contributions to the photon and photino propagators in $\mathcal{N}=1$ super Yang-Mills $\mathrm{U}(1)$ theory in the ordinary Feynman gauge. In section 3 we discuss, for later use, the construction by using the $\theta$-exact Seiberg-Witten map of a noncommutative U(1) theory with a noncommutative scalar field transforming under the adjoint representation. The oneloop propagators of the the ordinary fields of noncommutative $\mathcal{N}=2$ and 4 super Yang-Mills $\mathrm{U}(1)$ theories defined by using the $\theta$-exact Seiberg-Witten map are worked out in sections 4 and 5 in the ordinary Feynman gauge. In sections 6 and 7 we use a noncommutative Feynman gauge to quantize $\mathcal{N}=1$ super Yang-Mills $\mathrm{U}(1)$ theory and compute the oneloop photon propagator. Sections 6 and 7 are introduced to analyze the dependence on the gauge-fixing term of the UV and noncommutative IR contributions found in previous sections. The overall discussion of our results is carried out in section 8 . We also include several appendices which are needed to complete properly the analysis and computations carried out in the body of the paper.

\section{Noncommutative $\mathcal{N}=1$ SYM U(1) theory and the $\theta$-exact SW map}

The noncommutative field content of the noncommutative U(1) super Yang-Mills theory in the Wess-Zumino gauge is the noncommutative gauge field $A_{\mu}$, its supersymmetric fermion partner $\Lambda_{\alpha}$ and the noncommutative SUSY-auxiliary field $D^{(n c)}$. The ordinary/commutative counterparts of $A_{\mu}, \Lambda_{\alpha}$ and $D^{(n c)}$ will be denoted by $a_{\mu}$ (photon), $\lambda_{\alpha}$ 
(photino) and $D$, respectively. Regarding dotted and undotted fermions and $\sigma^{\alpha \dot{\alpha}}$ traces, we shall follow the conventions of [20].

In terms of the noncommutative fields and in the Wess-Zumino gauge the action of $\mathrm{U}(1)$ super Yang-Mills theory reads

$$
S_{\mathcal{N}=1}=\int-\frac{1}{4} F_{\mu \nu} \star F^{\mu \nu}+i \bar{\Lambda}_{\dot{\alpha}} \bar{\sigma}^{\mu \dot{\alpha} \alpha} \mathcal{D}_{\mu}[A] \Lambda_{\alpha}+\frac{1}{2} D^{(n c)} D^{(n c)},
$$

where $F_{\mu \nu}=\partial_{\mu} A_{\nu}-\partial_{\nu} A_{\mu}-i\left[A_{\mu} \stackrel{\star}{,} A_{\nu}\right]$ and $\mathcal{D}_{\mu}[A] \Lambda_{\alpha}=\partial_{\mu} \Lambda_{\alpha}-i\left[A_{\mu} \stackrel{\star}{,} \Lambda_{\alpha}\right]$.

The above action $\mathrm{S}_{\mathcal{N}=1}(2.1)$, is invariant under the following noncommutative supersymmetry transformations

$$
\begin{aligned}
\delta_{\xi} \Lambda_{\alpha} & =-i D^{(n c)} \xi_{\alpha}-e^{-1}\left(\sigma^{\mu} \bar{\sigma}^{\nu}\right)_{\alpha}{ }^{\beta} \xi_{\beta} F_{\mu \nu} \\
\delta_{\xi} A^{\mu} & =i e\left(\xi \sigma^{\mu} \bar{\Lambda}-\Lambda \sigma^{\mu} \bar{\xi}\right) \\
\delta_{\xi} D^{(n c)} & =\left(\xi \sigma^{\mu} \mathcal{D}_{\mu} \bar{\Lambda}-\mathcal{D}_{\mu} \Lambda \sigma^{\mu} \bar{\xi}\right) .
\end{aligned}
$$

These supersymmetry transformations close on translations modulo noncommutative gauge transformations and tell us that supersymmetry is linearly realized on the noncommutative fields - see [19] for further discussion. The action in (2.1), is also invariant under noncommutative U(1) transformations, which in the noncommutative BRST form read

$$
s_{\mathrm{NC}} \Lambda_{\alpha}=-i\left[\Lambda_{\alpha} \stackrel{\star}{,}\right], \quad s_{\mathrm{NC}} A_{\mu}=\partial_{\mu} \Omega-i\left[A_{\mu} \stackrel{\star}{,} \Omega\right], \quad s_{\mathrm{NC}} \Omega=i \Omega \star \Omega,
$$

with $\Omega$ being the noncommutative U(1) ghost field. The above action $S_{\mathcal{N}=1}$ can be expressed in terms of ordinary fields, $a_{\mu}, \lambda_{\alpha}$ and $D$, by means of the SW map. The resulting functional is invariant under ordinary $\mathrm{U}(1)$ BRST transformations:

$$
s \lambda_{\alpha}=-i\left[\lambda_{\alpha}, \omega\right], \quad s a_{\mu}=\partial_{\mu} \omega, \quad s \omega=0,
$$

where $\omega$ is the ordinary $\mathrm{U}(1)$ ghost field. Indeed, the SW map maps ordinary BRST orbits into the noncommutative BRST orbits.

The $\theta$-exact SW map for $F_{\mu \nu}$ has been worked out in [15] up to the three ordinary $\mathrm{U}(1)$ gauge fields $a_{\mu}$. It reads

$$
F_{\mu \nu}\left(e \cdot a_{\mu}, \theta^{\mu \nu}\right)=e f_{\mu \nu}+F_{\mu \nu}^{e^{2}}+F_{\mu \nu}^{e^{3}}+\mathcal{O}\left(e^{4}\right),
$$

where, up to the $e^{2}$ order, the gauge field strength SW map $F_{\mu \nu}^{e^{2}}$ expansion is fairly universal $[15-17]$

$$
F_{\mu \nu}^{e^{2}}=e^{2} \theta^{i j}\left(f_{\mu i} \star_{2} f_{\nu j}-a_{i} \star_{2} \partial_{j} f_{\mu \nu}\right) .
$$

The $e^{3}$ order SW map for the gauge field strength from [15] in that case reads

$$
\begin{aligned}
F_{\mu \nu}^{e^{3}}(x)= & \frac{e^{3}}{2} \theta^{i j} \theta^{k l}\left(\left[f_{\mu k} f_{\nu i} f_{l j}\right]_{\star_{3^{\prime}}}+\left[f_{\nu l} f_{\mu i} f_{k j}\right]_{\star_{3^{\prime}}}-\left[f_{\nu l} a_{i} \partial_{j} f_{\mu k}\right]_{\star_{3^{\prime}}}-\left[f_{\mu k} a_{i} \partial_{j} f_{\nu l}\right]_{\star_{3^{\prime}}}\right. \\
& -\left[a_{k} \partial_{l}\left(f_{\mu i} f_{\nu j}\right)\right]_{\star_{3^{\prime}}}+\left[a_{i} \partial_{j} a_{k} \partial_{l} f_{\mu \nu}\right]_{\star_{3^{\prime}}}+\left[\partial_{l} f_{\mu \nu} a_{i} \partial_{j} a_{k}\right]_{\star_{3^{\prime}}} \\
& \left.+\left[a_{k} a_{i} \partial_{l} \partial_{j} f_{\mu \nu}\right]_{\star_{3^{\prime}}}-\frac{1}{2}\left(\left[a_{i} \partial_{k} a_{j} \partial_{l} f_{\mu \nu}\right]_{\star_{3^{\prime}}}+\left[\partial_{l} f_{\mu \nu} a_{i} \partial_{k} a_{j}\right]_{\star_{3^{\prime}}}\right)\right) .
\end{aligned}
$$


The generalized star products relevant for this work are defined as follows [17, 18]

$$
\begin{aligned}
\left(f \star_{2} g\right)(x) & =\int e^{-i(p+q) x} \tilde{f}(p) \tilde{g}(q) f_{\star_{2}}(p, q), \\
{[f g h]_{\star_{3^{\prime}}}(x) } & =\int e^{-i(p+q+k) x} \tilde{f}(p) \tilde{g}(q) \tilde{h}(k) f_{\star_{3}}(p, q, k), \\
{[f g h]_{\mathcal{M}_{(\mathrm{I})}}(x) } & =\int e^{-i(p+q+k) x} \tilde{f}(p) \tilde{g}(q) \tilde{h}(k) f_{(\mathrm{I})}(p, q, k),
\end{aligned}
$$

with

$$
\begin{aligned}
f_{\star_{2}}(p, q) & =\frac{\sin \frac{p \theta q}{2}}{\frac{p \theta q}{2}}, \\
f_{\star_{3^{\prime}}}(p, q, k) & =\frac{\cos \left(\frac{p \theta q}{2}+\frac{p \theta k}{2}-\frac{q \theta k}{2}\right)-1}{\left(\frac{p \theta q}{2}+\frac{p \theta k}{2}-\frac{q \theta k}{2}\right) \frac{q \theta k}{2}}-\frac{\cos \left(\frac{p \theta q}{2}+\frac{p \theta k}{2}+\frac{q \theta k}{2}\right)-1}{\left(\frac{p \theta q}{2}+\frac{p \theta k}{2}+\frac{q \theta k}{2}\right) \frac{q \theta k}{2}} \\
f_{(\mathrm{I})}(p, q, k) & =\frac{1}{p \theta q}\left(f_{\star_{3^{\prime}}}[p, q,-(p+q+k)]-f_{\star_{3^{\prime}}}[p, q, k]\right) .
\end{aligned}
$$

The $\theta$-exact SW map for $\mathcal{D}_{\mu}[A] \Lambda_{\alpha}$ up to the two ordinary fields $a_{\mu}$ can be retrieved from the expression for $F_{\mu \nu}$ in (2.5), (2.6) and (2.7) as explained in the appendix A:

$$
\mathcal{D}_{\mu}[A] \Lambda_{\alpha}=\partial_{\mu} \lambda_{\alpha}+\mathcal{D}_{\mu \alpha}^{e}[e \cdot a, \lambda]+\mathcal{D}_{\mu \alpha}^{e^{2}}[e \cdot a, \lambda]+\mathcal{O}\left(e^{3}\right),
$$

where

$$
\mathcal{D}_{\mu \alpha}^{e}[e \cdot a, \lambda]=-e \theta^{i j}\left(f_{\mu i} \star_{2} \partial_{j} \lambda_{\alpha}+a_{i} \star_{2} \partial_{j} \partial_{\mu} \lambda_{\alpha}\right),
$$

and

$$
\begin{aligned}
\mathcal{D}_{\mu \alpha}^{e^{2}[e \cdot a, \lambda]=} & \frac{e^{2}}{2} \theta^{i j} \theta^{k l}\left(-\left[f_{\mu k} \partial_{i} \lambda_{\alpha} f_{l j}\right]_{\star_{3^{\prime}}}-\left[\partial_{l} \lambda_{\alpha} f_{\mu i} f_{k j}\right]_{\star_{3^{\prime}}}+\left[\partial_{l} \lambda_{\alpha} a_{i} \partial_{j} f_{\mu k}\right]_{\star_{3^{\prime}}}\right. \\
& +\left[f_{\mu k} a_{i} \partial_{j} \partial_{l} \lambda_{\alpha}\right]_{\star_{3^{\prime}}}+\left[a_{k} \partial_{l}\left(f_{\mu i} \partial_{j} \lambda_{\alpha}\right)\right]_{\star_{3^{\prime}}}+\left[a_{i} \partial_{j} a_{k} \partial_{l} \partial_{\mu} \lambda_{\alpha}\right]_{\star_{3^{\prime}}}+\left[\partial_{l} \partial_{\mu} \lambda_{\alpha} a_{i} \partial_{j} a_{k}\right]_{\star_{3^{\prime}}} \\
& \left.+\left[a_{k} a_{i} \partial_{l} \partial_{j} \partial_{\mu} \lambda_{\alpha}\right]_{\star_{3^{\prime}}}-\frac{1}{2}\left(\left[a_{i} \partial_{k} a_{j} \partial_{l} \partial_{\mu} \lambda_{\alpha}\right]_{\star_{3^{\prime}}}+\left[\partial_{l} \partial_{\mu} \lambda_{\alpha} a_{i} \partial_{k} a_{j}\right]_{\star_{3^{\prime}}}\right)\right) .
\end{aligned}
$$

To compute the full one-loop photon two-point function, one also needs the SW maps for the $\bar{\Lambda}_{\dot{\alpha}}$ and $D$ fields. They read

$$
\begin{aligned}
\bar{\Lambda}_{\dot{\alpha}}=\bar{\lambda}_{\dot{\alpha}}-e \theta^{i j} a_{i} \star_{2} \partial_{j} \bar{\lambda}_{\dot{\alpha}}+\frac{e^{2}}{4} \theta^{i j} \theta^{k l}\left(\left[a_{i} \partial_{j}\left(a_{k} \partial_{l} \bar{\lambda}_{\dot{\alpha}}\right)\right]_{\star_{3^{\prime}}}\right. \\
\left.-\left[a_{i}\left(f_{j k} \partial_{l} \bar{\lambda}_{\dot{\alpha}}-a_{k} \partial_{l} \partial_{j} \bar{\lambda}_{\dot{\alpha}}\right)\right]_{\star_{3^{\prime}}}+\left[\partial_{j} \bar{\lambda}_{\dot{\alpha}} a_{k}\left(\partial_{l} a_{i}+f_{l i}\right)\right]_{\star_{3^{\prime}}}\right)+\mathcal{O}\left(e^{3}\right), \\
D^{(n c)}=D-e \theta^{i j} a_{i} \star_{2} \partial_{j} D+\frac{e^{2}}{4} \theta^{i j} \theta^{k l}\left(\left[a_{i} \partial_{j}\left(a_{k} \partial_{l} D\right)\right]_{\star_{3^{\prime}}}\right. \\
\left.-\left[a_{i}\left(f_{j k} \partial_{l} D-a_{k} \partial_{l} \partial_{j} D\right)\right]_{\star_{3^{\prime}}}+\left[\partial_{j} D a_{k}\left(\partial_{l} a_{i}+f_{l i}\right)\right]_{\star_{3^{\prime}}}\right)+\mathcal{O}\left(e^{3}\right) .
\end{aligned}
$$

Let us stress that the way we have constructed - by appropriate restriction of the SW map for the gauge-field - the SW map for $\Lambda_{\alpha}$ and $D^{(n c)}$ is very much in harmony with 
the idea that if supersymmetry and gauge symmetry are not to clash, the superpartners must have similar behavior with respect to the gauge group.

As discussed in [19] the noncommutative supersymmetric transformations in (2.2) can be understood as the push-forward under the SW map of the appropriate $\theta$-dependent supersymmetric transformations of the ordinary fields. Here we have worked out the $\theta$ exact expression for such deformed transformations up to the order $e^{2}$,

$$
\begin{aligned}
\delta_{\xi}^{(n c)} \lambda_{\alpha} & =\delta_{\xi}^{(0)} \lambda_{\alpha}+e \delta_{\xi}^{(1)} \lambda_{\alpha}+e^{2} \delta_{\xi}^{(2)} \lambda_{\alpha}+\mathcal{O}\left(e^{3}\right), \\
\delta_{\xi}^{(n c)} a_{\mu} & =\delta_{\xi}^{(0)} a_{\mu}+e \delta_{\xi}^{(1)} a_{\mu}+e^{2} \delta_{\xi}^{(2)} a_{\mu}+\mathcal{O}\left(e^{3}\right), \\
\delta_{\xi}^{(n c)} D & =\delta_{\xi}^{(0)} D+e \delta_{\xi}^{(1)} a_{\mu}+e^{2} \delta_{\xi}^{(2)} D+\mathcal{O}\left(e^{3}\right),
\end{aligned}
$$

where

$$
\begin{aligned}
\delta_{\xi}^{(0)} \lambda_{\alpha} & =-i D \xi_{\alpha}-\frac{1}{2}\left(\sigma^{\mu} \bar{\sigma}^{\nu}\right)_{\alpha}{ }^{\beta} \xi_{\beta} f_{\mu \nu} \\
\delta_{\xi}^{(0)} a^{\mu} & =i\left(\xi \sigma^{\mu} \bar{\lambda}-\lambda \sigma^{\mu} \bar{\xi}\right) \\
\delta_{\xi}^{(0)} D & =\left(\xi \sigma \partial_{\mu} \bar{\Lambda}-\partial_{\mu} \lambda \sigma^{\mu} \bar{\xi}\right) \\
\delta_{\xi}^{(1)} \lambda_{\alpha} & =-i D^{(1)} \xi_{\alpha}-\frac{1}{2 e^{2}}\left(\sigma^{\mu} \bar{\sigma}^{\nu}\right)_{\alpha}{ }^{\beta} \xi_{\beta} F_{\mu \nu}^{e^{2}}-\delta_{\xi}^{(0)} \Lambda_{\alpha}^{(1)} \\
\delta_{\xi}^{(1)} a^{\mu} & =i\left(\xi \sigma \bar{\Lambda}^{(1)}-\Lambda^{(1)} \sigma^{\mu} \bar{\xi}\right)-\delta_{\xi}^{(0)} A_{\mu}^{(1)} \\
\delta_{\xi}^{(1)} D & =\left(\xi \sigma^{\mu} \overline{\mathcal{D}}_{\mu}^{e}-\mathcal{D}_{\mu}^{e} \sigma^{\mu} \bar{\xi}\right)-\delta_{\xi}^{(0)} D^{(1)} \\
\delta_{\xi}^{(2)} \lambda_{\alpha} & =-i D^{(2)} \xi_{\alpha}-\frac{1}{2 e^{3}}\left(\sigma^{\mu} \bar{\sigma}^{\nu}\right)_{\alpha}{ }^{\beta} \xi_{\beta} F_{\mu \nu}^{e^{3}}-\delta_{\xi}^{(1)} \Lambda_{\alpha}^{(1)}-\delta_{\xi}^{(0)} \Lambda_{\alpha}^{(2)} \\
\delta_{\xi}^{(2)} a^{\mu} & =i\left(\xi \sigma \bar{\Lambda}^{(2)}-\Lambda^{(2)} \sigma^{\mu} \bar{\xi}\right)-\delta_{\xi}^{(1)} A_{\mu}^{(1)}-\delta_{\xi}^{(0)} A_{\mu}^{(2)} \\
\delta_{\xi}^{(2)} D & =\left(\xi \sigma^{\mu} \overline{\mathcal{D}}_{\mu}^{e^{2}}-\mathcal{D}_{\mu}^{e^{2}} \sigma^{\mu} \bar{\xi}\right)-\delta_{\xi}^{(1)} D^{(1)}-\delta_{\xi}^{(0)} D^{(2)}
\end{aligned}
$$

The reader shall find in the appendix A the values of objects in the previous equations that have not been given yet.

The $\theta$-exact deformed supersymmetry transformations given in (2.15) and (2.16) can be rightly called supersymmetry transformations since, as shown in [19], they close on translations modulo gauge transformations and, hence, they carry a representation of the supersymmetry algebra. However notice that these deformed supersymmetry transformations of the ordinary field do not realize the supersymmetry algebra linearly. Finally, since these supersymmetry transformations generate the noncommutative supersymmetry transformations of (2.2), we conclude that the total $\theta$-exact action (given explicitly in the next subsection) has to be invariant up to the second order in $e$, under the deformed supersymmetry transformations in (2.15).

\subsection{The action}

Now, substituting into (2.1), the Seiberg-Witten maps from (2.6), (2.10) and (2.13) and dropping any contribution of order $e^{3}$, one obtains the SYM U(1) action in terms of commutative fields:

$$
S=S^{\text {photon }}+S^{\text {photino }}+S^{\text {SUSY-auxiliary }}
$$


where

$$
\begin{aligned}
& S^{\text {photon }}=\int-\frac{1}{4} f_{\mu \nu} f^{\mu \nu}-\frac{e}{2} \theta^{i j} f^{\mu \nu}\left(f_{\mu i} \star_{2} f_{\nu j}-\frac{1}{4} f_{i j} \star_{2} f_{\mu \nu}\right) \\
& -\frac{e^{2}}{4} \theta^{i j} \theta^{k l}\left(\left(f_{\mu i} \star_{2} f_{\nu j}\right)\left(f_{k}^{\mu} \star_{2} f_{l}^{\nu}\right)-\left(f_{i j} \star_{2} f_{\mu \nu}\right)\left(f_{k}^{\mu} \star_{2} f^{\nu}{ }_{l}\right)\right. \\
& +2 f^{\mu \nu}\left(a_{i} \star_{2} \partial_{j}\left(f_{\mu k} \star_{2} f_{\nu l}\right)-\left[f_{\mu k} a_{i} \partial_{j} f_{\nu l}\right]_{\star_{3^{\prime}}}-\left[a_{i} f_{\mu k} \partial_{j} f_{\nu l}\right]_{\star_{3^{\prime}}}\right. \\
& \left.+\left[f_{\mu i} f_{\nu k} f_{j l}\right]_{\star_{3^{\prime}}}-\frac{1}{8}\left[f_{\mu \nu} f_{i k} f_{j l}\right]_{\star_{3^{\prime}}}\right) \\
& \left.+\frac{1}{8}\left(f^{\mu \nu} \star_{2} f_{i j}\right)\left(f_{k l} \star_{2} f_{\mu \nu}\right)+\frac{1}{2} \theta^{p q} f_{\mu \nu}\left[\partial_{i} f_{j k} f_{l p} \partial_{q} f_{\mu \nu}\right]_{\mathcal{M}_{(\mathrm{I})}}\right), \\
& S^{\text {photino }}=\int i \bar{\lambda}_{\dot{\alpha}} \bar{\sigma}^{\mu \dot{\alpha} \alpha} \partial_{\mu} \lambda_{\alpha} \\
& -i e \theta^{i j}\left(\bar{\lambda}_{\dot{\alpha}} \bar{\sigma}^{\mu \dot{\alpha} \alpha}\left[f_{\mu i} \star_{2} \partial_{j} \lambda_{\alpha}+a_{i} \star_{2} \partial_{j} \partial_{\mu} \lambda_{\alpha}\right]+a_{i} \star_{2} \partial_{j} \bar{\lambda}_{\dot{\alpha}} \bar{\sigma}^{\mu \dot{\alpha} \alpha} \partial_{\mu} \lambda_{\alpha}\right) \\
& -i \frac{e^{2}}{2} \theta^{i j} \theta^{k l}\left(\overline { \lambda } _ { \dot { \alpha } } \overline { \sigma } ^ { \mu \dot { \alpha } \alpha } \left(\left[f_{\mu k} \partial_{i} \lambda_{\alpha} f_{l j}\right]_{\star_{3^{\prime}}}+\left[\partial_{l} \lambda_{\alpha} f_{\mu i} f_{k j}\right]_{\star_{3^{\prime}}}-\left[\partial_{l} \lambda_{\alpha} a_{i} \partial_{j} f_{\mu k}\right]_{\star_{3^{\prime}}}\right.\right. \\
& -\left[f_{\mu k} a_{i} \partial_{j} \partial_{l} \lambda_{\alpha}\right]_{\star_{3^{\prime}}}-\left[a_{k} \partial_{l}\left(f_{\mu i} \partial_{j} \lambda_{\alpha}\right)\right]_{\star_{3^{\prime}}}-\left[a_{i} \partial_{j} a_{k} \partial_{l} \partial_{\mu} \lambda_{\alpha}\right]_{\star_{3^{\prime}}}-\left[\partial_{l} \partial_{\mu} \lambda_{\alpha} a_{i} \partial_{j} a_{k}\right]_{\star_{3^{\prime}}} \\
& \left.-\left[a_{k} a_{i} \partial_{l} \partial_{j} \partial_{\mu} \lambda_{\alpha}\right]_{\star_{3^{\prime}}}+\frac{1}{2}\left[a_{i} \partial_{k} a_{j} \partial_{l} \partial_{\mu} \lambda_{\alpha}\right]_{\star_{3^{\prime}}}+\frac{1}{2}\left[\partial_{l} \partial_{\mu} \lambda_{\alpha} a_{i} \partial_{k} a_{j}\right]_{\star_{3^{\prime}}}\right) \\
& -2 a_{i} \star_{2} \partial_{j} \bar{\lambda}_{\dot{\alpha}} \bar{\sigma}^{\mu \dot{\alpha} \alpha}\left(f_{\mu k} \star_{2} \partial_{l} \lambda_{\alpha}+a_{k} \star_{2} \partial_{l} \partial_{\mu} \lambda_{\alpha}\right)-\frac{1}{2}\left(\left[a_{i} \partial_{j}\left(a_{k} \partial_{l} \bar{\lambda}_{\dot{\alpha}}\right)\right]_{\star_{3^{\prime}}}\right. \\
& \left.\left.-\left[a_{i}\left(f_{j k} \partial_{l} \bar{\lambda}_{\dot{\alpha}}-a_{k} \partial_{l} \partial_{j} \bar{\lambda}_{\dot{\alpha}}\right)\right]_{\star_{3^{\prime}}}+\left[\partial_{j} \bar{\lambda}_{\dot{\alpha}} a_{k}\left(\partial_{l} a_{i}+f_{l i}\right)\right]_{\star_{3^{\prime}}}\right) \bar{\sigma}^{\mu \dot{\alpha} \alpha} \partial_{\mu} \lambda_{\alpha}\right)+\mathcal{O}\left(e^{3}\right),
\end{aligned}
$$

and

$$
\begin{aligned}
& S^{\text {SUSY-auxiliary }}=\int \frac{1}{2} D D+e \theta^{i j} D\left(a_{i} \star_{2} \partial_{j} D\right) \\
& +\frac{e^{2}}{4} \theta^{i j} \theta^{k l} D\left(\left[a_{i} \partial_{j}\left(a_{k} \partial_{l} D\right)\right]_{\star_{3^{\prime}}}-\left[a_{i}\left(f_{j k} \partial_{l} D-a_{k} \partial_{l} \partial_{j} D\right)\right]_{\star_{3^{\prime}}}+\left[\partial_{j} D a_{k}\left(\partial_{l} a_{i}+f_{l i}\right)\right]_{\star_{3^{\prime}}}\right) \\
& +\frac{e^{2}}{2} \theta^{i j} \theta^{k l}\left(a_{i} \star_{2} \partial_{j} D\right)\left(a_{k} \star_{2} \partial_{l} D\right)+\mathcal{O}\left(e^{3}\right) .
\end{aligned}
$$

First we note that, since the Feynman rules of the 3- and 4-photon self-coupling (2.18), are already given in previous papers [16] and [18], respectively, we shall not repeat them here. Photino-photon Feynman rules, obtained from (2.19), are given explicitly in the appendix C.

\subsection{The photon one-loop contributions to the photon polarization tensor}

Most generally speaking, the total photon one-loop 1PI two-point function $\Pi^{\mu \nu}(p)$ in the $\mathcal{N}=1,2,4$ SYM theory is the sum of the following contributions

$$
\Pi^{\mu \nu}(p)=\left(B^{\mu \nu}(p)+T^{\mu \nu}(p)\right)+n_{f}\left(P^{\mu \nu}(p)_{\mathrm{bub}}+P^{\mu \nu}(p)_{\mathrm{tad}}\right)+n_{s}\left(S^{\mu \nu}(p)_{\mathrm{bub}}+S^{\mu \nu}(p)_{\mathrm{tad}}\right),
$$


where $B^{\mu \nu}(p), T^{\mu \nu}(p), P^{\mu \nu}(p)_{\text {bub }}, P^{\mu \nu}(p)_{\text {tad }}, S^{\mu \nu}(p)_{\text {bub }}$ and $S^{\mu \nu}(p)_{\text {tad }}$ refer to the contributions from the photon bubble and tadpole, the photino bubble and tadpole, and the adjoint scalar bubble and tadpole diagrams, respectively. The last two diagrams appear only in the extended SUSY, of course. We use $n_{f}$ for the number of photinos (Weyl fermions) and $n_{s}$ for the number of real adjoint scalar bosons (one complex scalar is counted as two real scalars), which are uniquely determined by $\mathcal{N}=1,2,4$ SUSY.

Explicit computation revolves that each of these diagrams can be expressed as a linear combinations of five transverse tensor structures

$$
\begin{aligned}
& \Pi\left(B, T, P, S_{\mathrm{bub}}, S_{\mathrm{tad}}\right)^{\mu \nu}(p)=\frac{e^{2}}{(4 \pi)^{2}}\left\{\left[g^{\mu \nu} p^{2}-p^{\mu} p^{\nu}\right] \Pi_{1}\left(B_{1}, T_{1}, P_{1}, S_{1}^{\mathrm{bub}}, S_{1}^{\mathrm{tad}}\right)(p)\right. \\
& +(\theta p)^{\mu}(\theta p)^{\nu} \Pi_{2}\left(B_{2}, T_{2}, P_{2}, S_{2}^{\mathrm{bub}}, S_{2}^{\mathrm{tad}}\right)(p) \\
& +\left[g^{\mu \nu}(\theta p)^{2}-(\theta \theta)^{\mu \nu} p^{2}+p^{\{\mu}(\theta \theta p)^{\nu\}}\right] \Pi_{3}\left(B_{3}, T_{3}, P_{3}, S_{3}^{\mathrm{bub}}, S_{3}^{\mathrm{tad}}\right)(p) \\
& +\left[(\theta \theta)^{\mu \nu}(\theta p)^{2}+(\theta \theta p)^{\mu}(\theta \theta p)^{\nu}\right] \Pi_{4}\left(B_{4}, T_{4}, P_{4}, S_{4}^{\mathrm{bub}}, S_{4}^{\mathrm{tad}}\right)(p) \\
& \left.+(\theta p)^{\{\mu}(\theta \theta \theta p)^{\nu\}} \Pi_{5}\left(B_{5}, T_{5}, P_{5}, S_{5}^{\mathrm{bub}}, S_{5}^{\mathrm{tad}}\right)(p)\right\} .
\end{aligned}
$$

The sum (2.21) can be expressed, in the language of the five tensor decomposition (2.22), as ${ }^{1}$

$$
\Pi_{i}(p)=B_{i}(p)+T_{i}(p)+n_{f} P_{i}(p)+n_{s}\left(S_{i}^{\mathrm{bub}}(p)+S_{i}^{\mathrm{tad}}(p)\right) .
$$

In the subsequent sections we are going to compute and give the coefficients $B_{i}(p), T_{i}(p)$, $P_{i}(p), S_{i}^{\mathrm{bub}}(p)$ and $S_{i}^{\mathrm{tad}}(p)$ in detail via equations (2.25), (2.27), (2.32), (3.6) and (3.12), respectively.

For the $\mathcal{N}=1$ theory $n_{f}=1, n_{s}=0$, thus we have

$$
\Pi_{i}^{\mathcal{N}=1}(p)=B_{i}(p)+T_{i}(p)+P_{i}(p) .
$$

In this section we are going to show that all quadratic IR divergences cancel in each of the $\Pi_{i}^{\mathcal{N}}=1$ 's, then we extend our results to the $\mathcal{N}=2,4$ theories as well.

We choose one specific set of four (five in the sections 6 and 7) nonplanar/special function integrals $T_{0}, I_{K}^{0}, I_{K}^{1}$ and $I_{H}$ alongside the usual planar/commutative UV divergent integrals to express all loop diagrams/coefficients in this article. This decomposition enjoys the advantage that each nonplanar integral bear distinctive asymptotic behavior in the IR regime: $T_{0}$ carries all the quadratic IR divergence $(\theta p)^{-2}$, with a pre-factor -2 , while $I_{K}^{0}$ and $I_{K}^{1}$ contain the dual logarithmic noncommutative IR divergence $\ln \left(p^{2}(\theta p)^{2}\right)$, with prefactors $-1 / 2$ and $-1 / 12$, respectively. The last integral $I_{H}$ is finite at the IR limit. Further details of these integrals are given in the appendix B.

\footnotetext{
${ }^{1}$ As we will see soon, the photino tadpole diagram vanishes, so we can simply denote $P^{\mu \nu}(p)=P^{\mu \nu}(p)_{\text {bub }}$ and consequently $P_{i}(p)=P_{i}(p)_{\text {bub }}$.
} 


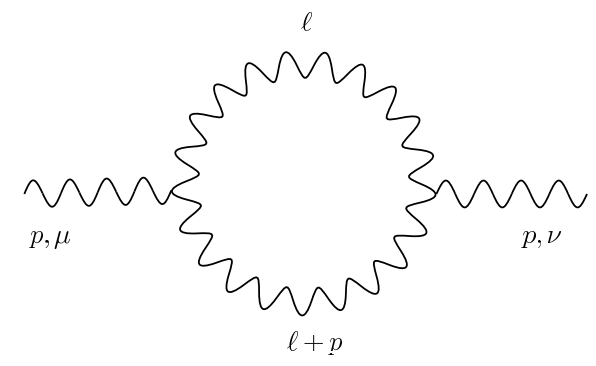

Figure 1. Three-photon bubble contribution to the photon two-point function $B^{\mu \nu}(p)$.

\subsubsection{The photon-bubble diagram}

From the photon bubble diagram figure 1 we obtain the following loop-coefficients [16]

$$
\begin{aligned}
B_{1}= & \left.\frac{2 D^{2}-9 D+8}{D-1}\left(4 \pi \mu^{2}\right)^{2-\frac{D}{2}}\left(p^{2}\right)^{\frac{D}{2}-1} \Gamma\left(2-\frac{D}{2}\right) \mathrm{B}\left(\frac{D}{2}-1, \frac{D}{2}-1\right)\right|_{D \rightarrow 4-\epsilon} \\
& -16 I_{K}^{1}-4 I_{H} \\
& +3 \operatorname{tr} \theta \theta \frac{p^{2}}{(\theta p)^{2}} \frac{1}{2}\left(\left.2\left(4 \pi \mu^{2}\right)^{2-\frac{D}{2}}\left(p^{2}\right)^{\frac{D}{2}-1} \Gamma\left(2-\frac{D}{2}\right) \mathrm{B}\left(\frac{D}{2}-1, \frac{D}{2}-1\right)\right|_{D \rightarrow 4-\epsilon}\right. \\
& \left.-4 I_{K}^{0}-4 I_{H}\right) \\
& +(\theta \theta p)^{2} \frac{p^{2}}{(\theta p)^{4}}\left(\left.4\left(4 \pi \mu^{2}\right)^{2-\frac{D}{2}}\left(p^{2}\right)^{\frac{D}{2}-1} \Gamma\left(2-\frac{D}{2}\right) \mathrm{B}\left(\frac{D}{2}-1, \frac{D}{2}-1\right)\right|_{D \rightarrow 4-\epsilon}\right. \\
& \left.-8 I_{K}^{0}-4 I_{H}\right), \\
B_{2}= & \frac{1}{(\theta p)^{2}}\left(\left.2(D-2)\left(4 \pi \mu^{2}\right)^{2-\frac{D}{2}}\left(p^{2}\right)^{\frac{D}{2}-1} \Gamma\left(2-\frac{D}{2}\right) \mathrm{B}\left(\frac{D}{2}-1, \frac{D}{2}-1\right)\right|_{D \rightarrow 4-\epsilon}\right. \\
& -\frac{16}{3} T_{0}+p^{2}\left(48 I_{K}^{1}-16 I_{K}^{0}-4 I_{H}\right) \\
& +\frac{1}{2} \operatorname{tr} \theta \theta \frac{p^{4}}{(\theta p)^{2}}\left(-\left.4\left(4 \pi \mu^{2}\right)^{2-\frac{D}{2}}\left(p^{2}\right)^{\frac{D}{2}-2} \Gamma\left(2-\frac{D}{2}\right) \mathrm{B}\left(\frac{D}{2}-1, \frac{D}{2}-1\right)\right|_{D \rightarrow 4-\epsilon}\right. \\
& \left.\left.+8 I_{K}^{0}+8 I_{H}\right)\right), \\
B_{3}= & \frac{1}{(\theta p)^{2}}\left(\left.2\left(4 \pi \mu^{2}\right)^{2-\frac{D}{2}}\left(p^{2}\right)^{\frac{D}{2}-1} \Gamma\left(2-\frac{D}{2}\right) \mathrm{B}\left(\frac{D}{2}-1, \frac{D}{2}-1\right)\right|_{D \rightarrow 4-\epsilon}\right. \\
& \left.+\frac{16}{3} T_{0}-p^{2}\left(4 I_{K}^{0}+8 I_{H}\right)\right),
\end{aligned}
$$




$$
\begin{aligned}
B_{4}= & \frac{p^{2}}{(\theta p)^{4}}\left(-\left.4\left(4 \pi \mu^{2}\right)^{2-\frac{D}{2}}\left(p^{2}\right)^{\frac{D}{2}-2} \Gamma\left(2-\frac{D}{2}\right) \mathrm{B}\left(\frac{D}{2}-1, \frac{D}{2}-1\right)\right|_{D \rightarrow 4-\epsilon}\right. \\
& \left.+\frac{16}{3} T_{0}+8 I_{K}^{0}+4 I_{H}\right), \\
B_{5}= & \frac{p^{2}}{(\theta p)^{4}}\left(\left.4\left(4 \pi \mu^{2}\right)^{2-\frac{D}{2}}\left(p^{2}\right)^{\frac{D}{2}-2} \Gamma\left(2-\frac{D}{2}\right) \mathrm{B}\left(\frac{D}{2}-1, \frac{D}{2}-1\right)\right|_{D \rightarrow 4-\epsilon}\right. \\
& \left.-8 I_{K}^{0}-4 I_{H}\right) .
\end{aligned}
$$

Extracting the divergent parts from each of the $B_{i}$ 's

$$
\begin{aligned}
& B_{1}(p)=+\left(\frac{4}{3}+3 \frac{p^{2}(\operatorname{tr} \theta \theta)}{(\theta p)^{2}}+4 \frac{p^{2}(\theta \theta p)^{2}}{(\theta p)^{4}}\right)\left(\frac{2}{\epsilon}+\ln \left(\mu^{2}(\theta p)^{2}\right)\right)+\text { finite terms } \\
& B_{2}(p)=+2 \frac{p^{2}}{(\theta p)^{2}}\left(2-\frac{p^{2}(\operatorname{tr} \theta \theta)}{(\theta p)^{2}}\right)\left(\frac{2}{\epsilon}+\ln \left(\mu^{2}(\theta p)^{2}\right)\right)+\frac{32}{3} \frac{1}{(\theta p)^{4}}+\text { finite terms } \\
& B_{3}(p)=+2 \frac{p^{2}}{(\theta p)^{2}}\left(\frac{2}{\epsilon}+\ln \left(\mu^{2}(\theta p)^{2}\right)\right)-\frac{32}{3} \frac{1}{(\theta p)^{4}}+\text { finite terms, } \\
& B_{4}(p)=-4 \frac{p^{4}}{(\theta p)^{4}}\left(\frac{2}{\epsilon}+\ln \left(\mu^{2}(\theta p)^{2}\right)\right)-\frac{32}{3} \frac{p^{2}}{(\theta p)^{6}}+\text { finite terms, } \\
& B_{5}(p)=+4 \frac{p^{4}}{(\theta p)^{4}}\left(\frac{2}{\epsilon}+\ln \left(\mu^{2}(\theta p)^{2}\right)\right)+\text { finite terms }
\end{aligned}
$$

we observe the presence of the UV plus logarithmic IR divergences in all of them. The logarithmic IR divergences from both planar and nonplanar sources in the bubble diagram appear to have identical coefficient and combine into a single $\ln \left(\mu^{2}(\theta p)^{2}\right)$ term, confirming the expected UV/IR mixing. The quadratic IR divergence, on the other hand, exists only in the $B_{2,3,4}$ terms.

\subsubsection{The photon-tadpole diagram}

From tadpole figure 2 we obtain the same tensor structure as from the photon bubble diagram (figure 1) with the following loop-coefficients $T_{i}(p)$ :

$$
\begin{aligned}
& T_{1}(p)=T_{5}(p)=0 \\
& T_{2}(p)=-\frac{32}{3} \frac{1}{(\theta p)^{2}} T_{0}=\frac{64}{3} \frac{1}{(\theta p)^{4}}, \\
& T_{3}(p)=-\frac{16}{3} \frac{1}{(\theta p)^{2}} T_{0}=\frac{32}{3} \frac{1}{(\theta p)^{4}} \\
& T_{4}(p)=-\frac{16}{3} \frac{1}{(\theta p)^{4}} T_{0}=\frac{32}{3} \frac{p^{2}}{(\theta p)^{6}} .
\end{aligned}
$$

We notice immediately the absence of UV plus logarithmic divergent terms contrary to the photon-bubble diagram results (2.26). In addition, the tadpole diagram produces no finite terms either, and the quadratic IR are again present in the second, third and fourth term! 


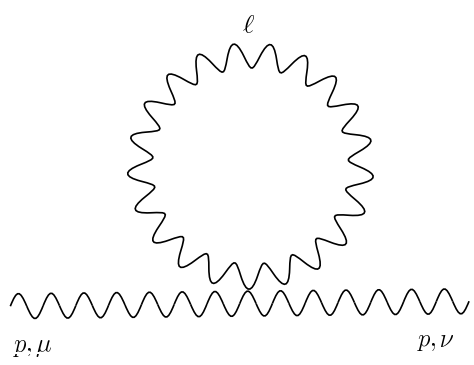

Figure 2. Four-photon tadpole contribution to the photon two-point function $T^{\mu \nu}(p)$.

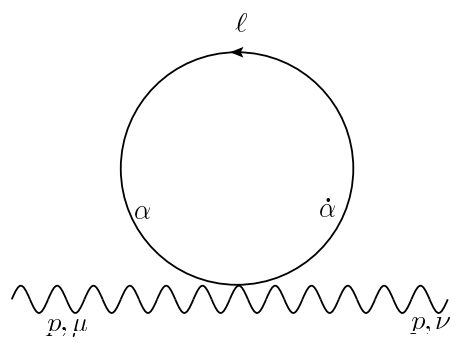

Figure 3. Photon-2photinos tadpole contribution to the photon two-point function $P_{\operatorname{tad}}^{\mu \nu}(p)$.

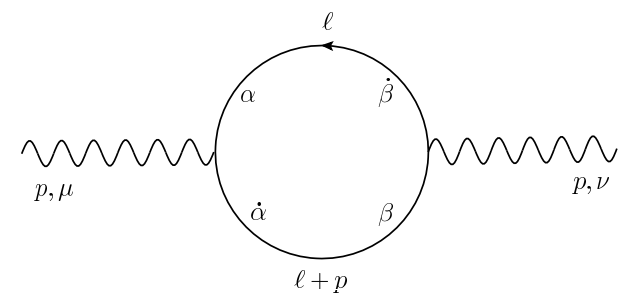

Figure 4. Photon-photino bubble contribution to the photon two-point function: $P_{\text {bub }}^{\mu \nu}(p)$.

\subsection{The photino one-loop contributions to the photon polarization tensor}

The photino sector contains two diagrams: photino tadpole figure 3 and photino bubble figure 4. We are going to see below that only the latter contributes to the photon polarization tensor.

\subsubsection{The photino-tadpole diagram}

The photino-tadpole contribution is computed using vertex (C.4). It produces only the quadratic IR divergent terms which cancel each other internally, thus giving vanishing contribution to the photon polarization tensor

$$
\begin{aligned}
P_{\text {tad }}^{\mu \nu}(p) & =-\left.\mu^{4-D} \int \frac{d^{D} \ell}{(2 \pi)^{D}} \frac{1}{\ell^{2}} V_{\mu_{1}}^{e^{2 \mu \nu}}\left[\ell,-p, p, \ell ; \theta^{i j}\right] \ell_{\mu_{2}} \operatorname{Tr}\left(\bar{\sigma}^{\mu_{1}} \sigma^{\mu_{2}}\right)\right|_{D \rightarrow 4} \\
& =-\frac{e^{2}}{3 \pi^{2}} \frac{(\theta p)^{\mu}(\theta p)^{\nu}}{(\theta p)^{4}}(4-2-2)=0 .
\end{aligned}
$$




\subsubsection{The photino-bubble diagram}

Taking the photino-photon Feynman rules from appendix $\mathrm{C}$ we obtain the following photino-bubble contribution to the photon polarization tensor, $P_{\text {bub }}^{\mu \nu}(p)$, from figure 4 :

$$
\begin{aligned}
P_{\mathrm{bub}}^{\mu \nu}(p)= & -\mu^{4-D} \int \frac{d^{D} \ell}{(2 \pi)^{D}} \frac{1}{\ell^{2}(\ell+p)^{2}} \\
& \cdot V_{\mu_{1}}^{e \mu}\left[\ell+p, p, \ell ; \theta^{i j}\right] \ell_{\mu_{2}} V_{\mu_{3}}^{e \nu}\left[\ell,-p, \ell+p ; \theta^{i j}\right](\ell+p)_{\mu_{4}} \operatorname{Tr}\left(\bar{\sigma}^{\mu_{1}} \sigma^{\mu_{2}} \bar{\sigma}^{\mu_{3}} \sigma^{\mu_{4}}\right) .
\end{aligned}
$$

Taking into account the trace

$$
\operatorname{Tr}\left(\bar{\sigma}^{\mu_{1}} \sigma^{\mu_{2}} \bar{\sigma}^{\mu_{3}} \sigma^{\mu_{4}}\right)=2\left(\eta^{\mu_{1} \mu_{2}} \eta^{\mu_{3} \mu_{4}}-\eta^{\mu_{1} \mu_{3}} \eta^{\mu_{2} \mu_{4}}+\eta^{\mu_{1} \mu_{4}} \eta^{\mu_{2} \mu_{3}}-i \epsilon^{\mu_{1} \mu_{2} \mu_{3} \mu_{4}}\right),
$$

and that $P_{\mathrm{bub}}^{\mu \nu}(p)$ cannot have, at the end of the day, contributions depending on $\epsilon^{\mu_{1} \mu_{2} \mu_{3} \mu_{4}}$, one arrives at

$$
\begin{aligned}
P_{\mathrm{bub}}^{\mu \nu}(p)= & -\mu^{4-D} \int \frac{d^{D} \ell}{(2 \pi)^{D}} \frac{1}{\ell^{2}(\ell+p)^{2}} \\
& \cdot\left[V_{\mu_{1}}^{e \mu}\left[\ell+p, p, \ell ; \theta^{i j}\right] \ell^{\mu_{1}} V_{\mu_{3}}^{e \nu}\left[\ell,-p, \ell+p ; \theta^{i j}\right](\ell+p)^{\mu_{3}}\right. \\
& +V_{\mu_{1}}^{e \mu}\left[\ell+p, p, \ell ; \theta^{i j}\right](\ell+p)^{\mu_{1}} V_{\mu_{3}}^{e \nu}\left[\ell,-p, \ell+p ; \theta^{i j}\right] \ell^{\mu_{3}} \\
& \left.-V_{\mu_{1}}^{e \mu}\left[\ell+p, p, \ell ; \theta^{i j}\right] \eta^{\mu_{1} \mu_{3}} V_{\mu_{3}}^{e \nu}\left[\ell,-p, \ell+p ; \theta^{i j}\right] \ell \cdot(\ell+p)\right] .
\end{aligned}
$$

After some amount of computations we find that only first two of the general five-terms structure (2.22) survive in $D=4$ dimensions:

$$
\begin{aligned}
& P_{1}(p)=-\left.2 \frac{D-2}{D-1}\left(4 \pi \mu^{2}\right)^{2-\frac{D}{2}}\left(p^{2}\right)^{\frac{D}{2}-2} \Gamma\left(2-\frac{D}{2}\right) \mathrm{B}\left(\frac{D}{2}-1, \frac{D}{2}-1\right)\right|_{D \rightarrow 4-\epsilon}+16 I_{K}^{1}, \\
& P_{2}(p)=16 T_{0}+8 p^{2}\left(I_{K}^{0}-6 I_{K}^{1}\right), P_{3}(p)=P_{4}(p)=P_{5}(p)=0 .
\end{aligned}
$$

After inspecting the divergences in these two terms we also find that the first of the two, $P_{1}(p)$, contain the logarithmic UV/IR mixing terms, while $P_{2}(p)$ possesses only quadratic IR divergence and finite terms, as the dual $\mathrm{NC}$ logarithmic divergences from integrals $I_{K}^{0}$ and $I_{K}^{1}$ cancel each other.

Summing over (2.26), (2.27) and (2.32) one can see that the total quadratic IR divergences in all $\Pi_{i}$ 's are zero. The total UV divergences for the $\mathcal{N}=1$ theory are as follows

$$
\begin{aligned}
& \left.\Pi_{1}(p)\right|_{\mathrm{UV}}=\frac{p^{2}}{(\theta p)^{4}}\left(3(\operatorname{tr} \theta \theta)(\theta p)^{2}+4(\theta \theta p)^{2}\right)\left(\frac{2}{\epsilon}+\ln \left(\mu^{2}(\theta p)^{2}\right)\right), \\
& \left.\Pi_{2}(p)\right|_{\mathrm{UV}}=\frac{p^{2}}{(\theta p)^{2}}\left(2-\frac{p^{2}(\operatorname{tr} \theta \theta)}{(\theta p)^{2}}\right)\left(\frac{2}{\epsilon}+\ln \left(\mu^{2}(\theta p)^{2}\right)\right), \\
& \left.\Pi_{3}(p)\right|_{\mathrm{UV}}=\frac{p^{2}}{(\theta p)^{2}}\left(\frac{2}{\epsilon}+\ln \left(\mu^{2}(\theta p)^{2}\right)\right), \\
& \left.\Pi_{4}(p)\right|_{\mathrm{UV}}=-4 \frac{p^{4}}{(\theta p)^{4}}\left(\frac{2}{\epsilon}+\ln \left(\mu^{2}(\theta p)^{2}\right)\right), \\
& \left.\Pi_{5}(p)\right|_{\mathrm{UV}}=4 \frac{p^{4}}{(\theta p)^{4}}\left(\frac{2}{\epsilon}+\ln \left(\mu^{2}(\theta p)^{2}\right)\right) .
\end{aligned}
$$




\subsection{The one-loop SUSY-auxiliary field contributions to the photon propagator}

The free two-point function of the SUSY-auxiliary field $D$ reads

$$
\langle D(x) D(y)\rangle=\delta(x-y),
$$

hence, the integrals to be computed in dimensional regularization are of the type

$$
\int \frac{d^{D} \ell}{(2 \pi)^{D}} \frac{e^{i(\ell \theta p)}}{(\ell \theta p)^{n}}
$$

The integrals in (2.39) vanish in dimensional regularization and hence the SUSY-auxiliary field $D$ does not contribute to the one-loop photon propagator. Indeed, following [21], we first split the $D$-dimensional $\ell\left(\equiv \ell^{(D)}\right)$ into

$$
\ell^{(D)}=\ell^{(D-1)}+x \frac{\theta p}{|\theta p|},
$$

where $\ell^{(D-1)}$ belongs to $D-1$ dimensional space orthogonal to $\theta p$, and $|\theta p|>0$ is the modulus of $\theta p$ - recall that $\theta p$ is a space-like vector, since $\theta^{\mu 0}=0$. Then introduce the following definition of the dimensionally regularized integral in (2.39):

$$
\int \frac{d^{D} \ell}{(2 \pi)^{D}} \frac{e^{i \ell \theta p}}{(\ell \theta p)^{n}}=\int \frac{d^{(D-1)} \ell}{(2 \pi)^{(D-1)}} \lim _{\varepsilon \rightarrow 0} \int_{-\infty}^{+\infty} d x \frac{e^{i x}}{(x+i \varepsilon)^{n}} .
$$

However, in dimensional regularization — see [21]

$$
\int \frac{d^{(D-1)} \ell}{(2 \pi)^{(D-1)}}=0
$$

which in turn leads to the conclusion that the integral (2.39) vanishes under the dimensional regularization procedure.

It is not difficult to see that the argument above can be generalized to integrals with positive $\ell$ powers too, i.e.

$$
\int \frac{d^{D} \ell}{(2 \pi)^{D}} \ell^{\mu_{1}} \ldots \ell^{\mu_{2 n}} \frac{e^{i(\ell \theta p)}}{(\ell \theta p)^{n}}=0
$$

One can explicitly verify two special cases of the identity above

$$
\int \frac{d^{D} \ell}{(2 \pi)^{D}} f_{\star_{2}}(\ell, p)^{2}=\int \frac{d^{D} \ell}{(2 \pi)^{D}} \ell^{\mu} \ell^{\nu} f_{\star_{2}}(\ell, p)^{2}=0,
$$

using a generalization of the n-nested zero regulator method [18].

\subsection{The one-loop photino 1-PI two point function}

The photino self-energy consists two diagrams, a tadpole figure 5 and a bubble figure 6 . Explicit computation shows that the tadpole diagram figure 5 vanishes:

$$
\Sigma^{\dot{\alpha} \alpha}(p)_{\operatorname{tad}}=0
$$




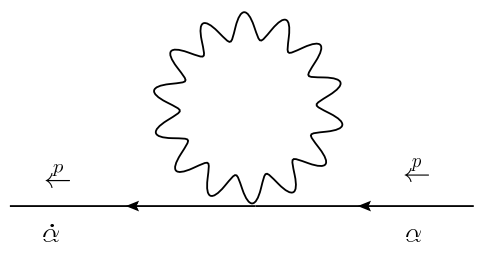

Figure 5. $\mathcal{N}=1$ photino-photon tadpole: $\Sigma^{\dot{\alpha} \alpha}(p)_{\operatorname{tad}}$.

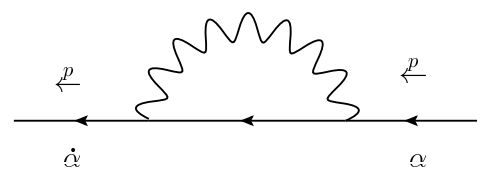

Figure 6. $\mathcal{N}=1$ photino-photon bubble: $\Sigma^{\dot{\alpha} \alpha}(p)_{\text {bub }}$.

The bubble diagram was computed in [16], which boils down to following expressions

$$
\Sigma^{\dot{\alpha} \alpha}(p)_{\mathrm{bub}}=-\frac{e^{2}}{(4 \pi)^{2}} \sigma_{\mu}^{\dot{\alpha} \alpha}\left[p^{\mu} N_{1}(p)+(\theta \theta p)^{\mu} N_{2}(p)\right]
$$

with

$$
\begin{aligned}
N_{1}(p)= & -\frac{1}{2}(\theta p)^{2} I_{H} \\
& +\operatorname{tr} \theta \theta \frac{p^{2}}{(\theta p)^{2}}\left(\left.\left(4 \pi \mu^{2}\right)^{2-\frac{D}{2}}\left(p^{2}\right)^{\frac{D}{2}-2} \Gamma\left(2-\frac{D}{2}\right) \mathrm{B}\left(\frac{D}{2}-1, \frac{D}{2}-1\right)\right|_{D \rightarrow 4-\epsilon}\right. \\
& \left.-\left(2 I_{K}^{0}+2 I_{H}\right)\right) \\
& +(\theta \theta p)^{2} \frac{p^{2}}{(\theta p)^{4}}\left(\left.2\left(4 \pi \mu^{2}\right)^{2-\frac{D}{2}}\left(p^{2}\right)^{\frac{D}{2}-2} \Gamma\left(2-\frac{D}{2}\right) \mathrm{B}\left(\frac{D}{2}-1, \frac{D}{2}-1\right)\right|_{D \rightarrow 4-\epsilon}\right. \\
& \left.-\left(4 I_{K}^{0}+2 I_{H}\right)\right),
\end{aligned}
$$

and

$$
N_{2}(p)=4 \frac{p^{2}}{(\theta p)^{2}} I_{H}
$$

One can easily notice the absence of the quadratic IR divergent integral $T_{0}$. The UV divergence can be expressed as follows

$$
\begin{aligned}
\left.\Sigma^{\dot{\alpha} \alpha}(p)_{\mathrm{bub}}\right|_{\mathrm{UV}} & =-\left.\frac{e^{2}}{(4 \pi)^{2}} \sigma_{\mu}^{\dot{\alpha} \alpha} p^{\mu} N_{1}(p)\right|_{\mathrm{UV}} \\
& =-\frac{e^{2}}{(4 \pi)^{2}} \sigma_{\mu}^{\dot{\alpha} \alpha} p^{\mu}\left(\operatorname{tr} \theta \theta \frac{p^{2}}{(\theta p)^{2}}+2(\theta \theta p)^{2} \frac{p^{2}}{(\theta p)^{4}}\right)\left(\frac{2}{\epsilon}+\ln \left(\mu^{2}(\theta p)^{2}\right)\right) .
\end{aligned}
$$




\section{Minimal action of the noncommutative adjoint scalar field}

It is commonly known that extended, $\mathcal{N}=2,4$, super YM theories contain not only fermion (photino) but also scalar bosons in the adjoint representation. These scalar bosons couple minimally to the gauge field, and their action for the real scalar is

$$
S^{\text {real }}=\frac{1}{2} \int \mathcal{D}^{\mu} \Phi \mathcal{D}_{\mu} \Phi,
$$

or

$$
S^{\text {complex }}=\int \mathcal{D}^{\mu} \Phi^{\dagger} \mathcal{D}_{\mu} \Phi,
$$

for the complex scalar. We study the minimal interacting scalar boson's contribution to 1-PI photon two point function as well as the scalar's own 1-PI two point function in this section. These results will be used for our discussion on $\mathcal{N}=2,4 \mathrm{SYM}$ in the subsequent sections.

It is straightforward to derive the SW map expansion of either $S^{\text {real }}$ or $S^{\text {complex }}$ using the method described in the appendix A

$$
\begin{aligned}
& S^{\text {real }}=\frac{1}{2} \int \mathcal{D}^{\mu} \Phi \mathcal{D}_{\mu} \Phi=\frac{1}{2} \int \partial^{\mu} \phi \partial_{\mu} \phi-2 e \theta^{i j} \partial^{\mu} \phi\left(f_{\mu i} \star_{2} \partial_{j} \phi+\frac{1}{4} f_{i j} \star_{2} \partial_{\mu} \phi\right)+ \\
& +e^{2} \theta^{i j} \theta^{k l}\left(\left(f_{\mu i} \star_{2} \partial_{j} \phi\right)\left(f_{k}^{\mu} \star_{2} \partial_{l} \phi\right)+\left(\partial_{\mu} \phi \star_{2} f_{i j}\right)\left(f_{k}^{\mu} \star_{2} \partial_{l} \phi\right)\right. \\
& +\partial^{\mu} \phi\left(\left[f_{\mu i} \partial_{l} \phi f_{i k}\right]_{\star_{3^{\prime}}}+\left[\partial_{l} \phi f_{\mu i} f_{i k}\right]_{\star_{3^{\prime}}}+\left[\partial_{l} \phi a_{i} \partial_{j} f_{\mu k}\right]_{\star_{3^{\prime}}}+\left[f_{\mu k} a_{i} \partial_{j} \partial_{l} \phi\right]_{\star_{3^{\prime}}}\right. \\
& +\left[a_{i} \partial_{j}\left(f_{\mu k} \partial_{l} \phi\right)\right]_{\star_{3^{\prime}}}-2 a_{i} \star_{2} \partial_{j}\left(f_{\mu k} \star_{2} \partial_{l} \phi\right)+\frac{1}{4}\left[\partial_{\mu} \phi f_{i l} f_{j k}\right]_{\star_{3^{\prime}}} \\
& \left.\left.+\frac{1}{8} f_{i j} \star_{2}\left(f_{k l} \star_{2} \partial_{\mu} \phi\right)+\frac{1}{2} \theta^{p q}\left[\partial_{i} f_{j k} f_{l p} \partial_{q} \partial_{\mu} \phi\right]_{\mathcal{M}_{(\mathrm{I})}}\right)\right) \text {, } \\
& S^{\text {complex }}=\int \mathcal{D}^{\mu} \Phi^{\dagger} \mathcal{D}_{\mu} \Phi=\int \partial_{\mu} \phi^{*} \partial^{\mu} \phi \\
& -e \theta^{i j}\left(\partial^{\mu} \phi^{*}\left(f_{\mu i} \star_{2} \partial_{j} \phi\right)+\left(f_{\mu i} \star_{2} \partial_{j} \phi^{*}\right) \partial^{\mu} \phi+\frac{1}{2}\left(\partial_{\mu} \phi^{*} \star_{2} f_{i j}\right) \partial^{\mu} \phi\right) \\
& +e^{2} \theta^{i j} \theta^{k l}\left(\left(f_{\mu i} \star_{2} \partial_{j} \phi^{*}\right)\left(f_{k}^{\mu} \star_{2} \partial_{l} \phi\right)+\frac{1}{2}\left(\left(f_{\mu i} \star_{2} \partial_{j} \phi^{*}\right)\left(f_{i j} \star_{2} \partial^{\mu} \phi\right)\right.\right. \\
& \left.+\left(f_{\mu i} \star_{2} \partial_{j} \phi\right)\left(f_{i j \star_{2}} \partial^{\mu} \phi^{*}\right)\right)+\frac{1}{2} \partial^{\mu} \phi^{*}\left(\left[f_{\mu i} \partial_{l} \phi f_{j k}\right]_{\star_{3^{\prime}}}+\left[\partial_{l} \phi f_{\mu i} f_{j k}\right]_{\star_{3^{\prime}}}\right) \\
& +\frac{1}{2}\left(\left[f_{\mu i} \partial_{l} \phi^{*} f_{j k}\right]_{\star_{3^{\prime}}}+\left[\partial_{l} \phi^{*} f_{\mu i} f_{j k}\right]_{\star_{3^{\prime}}}\right) \partial^{\mu} \phi+\frac{1}{2} \partial^{\mu} \phi^{*}\left(\left[\partial_{l} \phi a_{i} \partial_{j} f_{\mu k}\right]_{\star_{3^{\prime}}}\right. \\
& \left.+\left[f_{\mu k} a_{i} \partial_{j} \partial_{l} \phi\right]_{\star_{3^{\prime}}}+\left[a_{i} \partial_{j}\left(f_{\mu k} \partial_{l} \phi\right)\right]_{\star_{3^{\prime}}}-2 a_{i} \star_{2} \partial_{j}\left(f_{\mu k} \star_{2} \partial_{l} \phi\right)\right) \\
& +\frac{1}{2}\left(\left[\partial_{l} \phi^{*} a_{i} \partial_{j} f_{\mu k}\right]_{\star_{3^{\prime}}}+\left[f_{\mu k} a_{i} \partial_{j} \partial_{l} \phi^{*}\right]_{\star_{3^{\prime}}}+\left[a_{i} \partial_{j}\left(f_{\mu k} \partial_{l} \phi^{*}\right)\right]_{\star_{3^{\prime}}}\right. \\
& \left.-2 a_{i} \star_{2} \partial_{j}\left(f_{\mu k} \star_{2} \partial_{l} \phi^{*}\right)\right) \partial^{\mu} \phi+\frac{1}{4} \partial^{\mu} \phi^{*}\left[\partial_{\mu} \phi f_{i l} f_{j k}\right]_{\star_{3^{\prime}}}+\frac{1}{8}\left(\partial^{\mu} \phi^{*} \star_{2} f_{i j}\left(\partial_{\mu} \phi \star_{2} f_{k l}\right)\right. \\
& \left.+\frac{1}{4} \theta^{p q}\left(\partial^{\mu} \phi^{*}\left[\partial_{i} f_{j k} f_{l p} \partial_{q} \partial_{\mu} \phi\right]_{\mathcal{M}_{(\mathrm{I})}}-\partial_{q} \partial_{\mu} \phi^{*}\left[\partial_{i} f_{j k} f_{l p} \partial_{\mu} \phi\right]_{\mathcal{M}_{(\mathrm{I})}}\right)\right),
\end{aligned}
$$




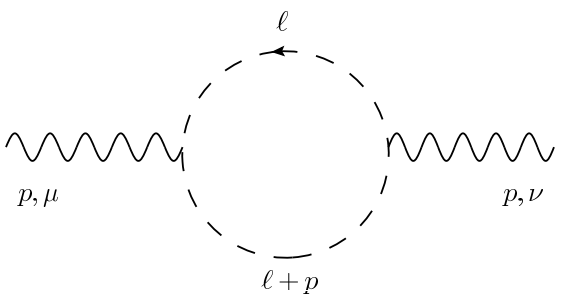

Figure 7. Photon-scalar bubble: $S^{\mu \nu}(p)_{\text {bub }}$.

with the product $\mathcal{M}_{(\mathrm{I})}$ being defined in [18]. One can show that

$$
S^{\text {complex }}\left(\phi=\frac{1}{\sqrt{2}}\left(\varphi_{1}+i \varphi_{2}\right)\right)=S^{\text {real }}\left(\varphi_{1}\right)+S^{\text {real }}\left(\varphi_{2}\right)
$$

if we express one complex scalar in terms of two real scalars. For this reason one complex scalar contribution to the photon 1-PI two point function is twice as one real scalar, while the photon contribution to the complex scalar two point function is the same as for the real scalar two point function. Thus, we shall compute only those for the real scalar field. The scalar-photon Feynman rules are given in the appendix D.

\subsection{Scalar one-loop contributions to the photon polarization tensor}

Like the photino sector, the adjoint scalar sector contains also two diagrams that contribute to the photon polarization tensor, the scalar-bubble diagram figure 7 and scalar-tadpole diagram figure 8 . They both follow the five tensor structure decomposition (2.22) and stay nonzero at the $D \rightarrow 4-\epsilon$ limit.

\subsubsection{The scalar-bubble diagram}

Using Feynman rule (D.1) and employing the dimensional regularization techniques we obtain the following loop-coefficielnts from the photon-scalar bubble diagram figure 7 :

$$
\begin{aligned}
S_{1}^{\mathrm{bub}}(p)= & -\left.\frac{1}{D-1}\left(4 \pi \mu^{2}\right)^{2-\frac{D}{2}}\left(p^{2}\right)^{\frac{D}{2}-2} \Gamma\left(2-\frac{D}{2}\right) \mathrm{B}\left(\frac{D}{2}-1, \frac{D}{2}-1\right)\right|_{D \rightarrow 4-\epsilon} \\
& -8 I_{K}^{1}+2 I_{K}^{0}, \\
S_{2}^{\mathrm{bub}}(p)= & -8 \frac{1}{(\theta p)^{2}} T_{0}=\frac{16}{(\theta p)^{4}}, S_{3}^{\mathrm{bub}}\left(p=4 \frac{1}{(\theta p)^{2}} T_{0}=-\frac{8}{(\theta p)^{4}},\right. \\
S_{4}^{\mathrm{bub}}(p)= & \frac{16}{3} \frac{p^{2}}{(\theta p)^{4}} T_{0}=-\frac{32}{3} \frac{p^{2}}{(\theta p)^{6}}, S_{5}^{\mathrm{bub}}(p)=0 .
\end{aligned}
$$

\subsubsection{The scalar-tadpole diagram}

Next, with Feynman rule (D.2) we compute the photon-scalar tadpole diagram in figure 8,

$$
S^{\mu \nu}(p)_{\operatorname{tad}}=\sum_{k^{\prime}=1}^{4} S_{k^{\prime}}^{\mu \nu}(p)_{\operatorname{tad}}=\frac{1}{2} \int \frac{d^{D} \ell}{(2 \pi)^{D}} \frac{i}{\ell^{2}} \sum_{k^{\prime}=1}^{4} S_{k^{\prime}}^{\mu \nu}(\ell, p,-p,-\ell) .
$$




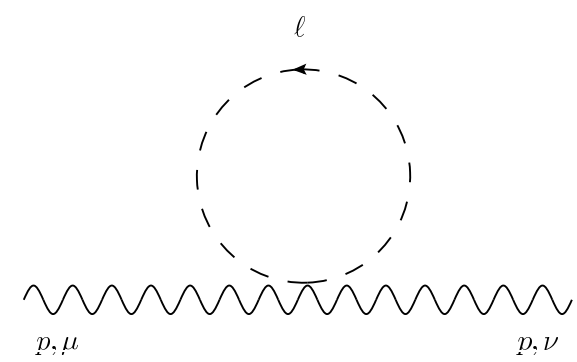

Figure 8. Photon-2scalars tadpole: $S^{\mu \nu}(p)_{\operatorname{tad}}$.

Starting with the first integral under dimensional regularization:

$$
\begin{aligned}
& S_{1^{\prime}}^{\mu \nu}(p)_{\operatorname{tad}}=-\left.e^{2} \int \frac{d^{D} \ell}{(2 \pi)^{D}} \frac{f_{\star_{2}}(\ell, p)}{\ell^{2}}\left((p \theta \ell)\left((p \theta \ell) g^{\mu \nu}-p^{\mu}(\theta \ell)^{\nu}-(\theta \ell)^{\mu} p^{\nu}\right)+p^{2}(\theta \ell)^{\mu}(\theta \ell)^{\nu}\right)\right|_{D \rightarrow 4} \\
& =\frac{e^{2}}{(4 \pi)^{2}} \frac{8}{3(\theta p)^{4}}\left[3\left(g^{\mu \nu}(\theta p)^{2}-(\theta \theta)^{\mu \nu} p^{2}+p^{\{\mu}(\theta \theta p)^{\nu\}}\right)+\frac{4 p^{2}}{(\theta p)^{2}}\left((\theta \theta)^{\mu \nu}(\theta p)^{2}+(\theta \theta p)^{\mu}(\theta \theta p)^{\nu}\right)\right],
\end{aligned}
$$

we obtained the IR result. To evaluate $S_{2^{\prime}}^{\mu \nu}(p)_{\operatorname{tad}}, S_{3^{\prime}}^{\mu \nu}(p)_{\operatorname{tad}}$ and $S_{4^{\prime}}^{\mu \nu}(p)_{\operatorname{tad}}$ we first need to establish the following identities:

$$
\begin{aligned}
f_{\star_{3^{\prime}}}(\ell, p,-p)=f_{\star_{3^{\prime}}}(\ell,-p, p)=f_{\star_{3^{\prime}}}(-\ell, p,-p)=f_{\star_{3^{\prime}}}(-\ell,-p, p) & =1, \\
f_{\star_{3^{\prime}}}(p,-p,-\ell)=f_{\star_{3^{\prime}}}(p,-p, \ell)=f_{\star_{3^{\prime}}}(-p, p,-\ell)=f_{\star_{3^{\prime}}}(-p, p, \ell) & =f_{\star_{2}}^{2}(\ell, p), \\
f_{\star_{3^{\prime}}}(-\ell, p,-p)+f_{\star_{3^{\prime}}}(p,-p,-\ell)-2 f_{\star_{2}}^{2}(\ell, p) & \sim-f_{\star_{2}}^{2}(\ell, p), \\
f_{\star_{3^{\prime}}}(p,-p,-\ell)+f_{\star_{3^{\prime}}}(-p, p,-\ell)-2 f_{\star_{2}}^{2}(\ell, p) & =0, \\
f_{\star_{3^{\prime}}}(\ell, p,-p)+f_{\star_{3^{\prime}}}(p,-p, \ell)-2 f_{\star_{2}}^{2}(\ell, p) & \sim-f_{\star_{2}}^{2}(\ell, p), \\
f_{\star_{3^{\prime}}}(p,-p, \ell)+f_{\star_{3^{\prime}}}(-p, p, \ell)-2 f_{\star_{2}}^{2}(\ell, p) & =0 .
\end{aligned}
$$

We then find the following pure IR divergent terms:

$$
\begin{aligned}
& S_{4^{\prime}}^{\mu \nu}(p)_{\mathrm{tad}}=-2 S_{2^{\prime}}^{\mu \nu}(p)_{\mathrm{tad}}=-2 S_{3^{\prime}}^{\mu \nu}(p)_{\mathrm{tad}}=-S_{2^{\prime}}^{\mu \nu}(p)_{\mathrm{tad}}-S_{3^{\prime}}^{\mu \nu}(p)_{\mathrm{tad}} \\
& =\left.e^{2} \int \frac{d^{D} \ell}{(2 \pi)^{D}} \frac{f_{\star_{2}}(\ell, p)}{\ell^{2}}\left((p \ell)\left((\theta p)^{\mu}(\theta \ell)^{\nu}+(\theta \ell)^{\mu}(\theta p)^{\nu}\right)-(p \theta \ell)\left(\ell^{\mu}(\theta p)^{\nu}+(\theta p)^{\mu} \ell^{\nu}\right)\right)\right|_{D \rightarrow 4} \\
& =\frac{e^{2}}{(4 \pi)^{2}} \frac{32}{3} \frac{(\theta p)^{\mu}(\theta p)^{\nu}}{(\theta p)^{4}}, \\
& S^{\mu \nu}(p)_{\mathrm{tad}}=S_{1^{\prime}}^{\mu \nu}(p)_{\mathrm{tad}} .
\end{aligned}
$$

Using (3.8) and (3.11) and by comparing with general tensor structure (2.22) we have found that from scalar-photon tadpole diagram only two terms survives:

$$
\begin{aligned}
& S_{1}^{\operatorname{tad}}(p)=S_{2}^{\operatorname{tad}}(p)=S_{5}^{\operatorname{tad}}(p)=0, \\
& S_{3}^{\operatorname{tad}}(p)=-4 \frac{1}{(\theta p)^{2}} T_{0}=\frac{8}{(\theta p)^{4}}, \quad S_{4}^{\operatorname{tad}}(p)=-\frac{16}{3} \frac{p^{2}}{(\theta p)^{4}} T_{0}=\frac{32}{3} \frac{p^{2}}{(\theta p)^{6}} .
\end{aligned}
$$




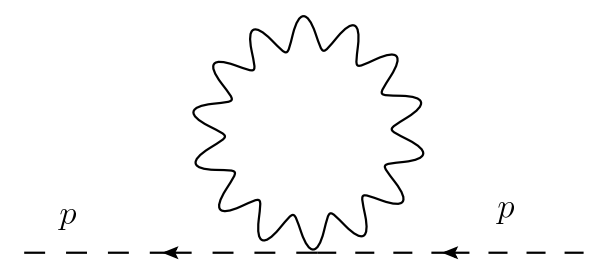

Figure 9. The scalar-photon tadpole: $\Sigma_{(\phi)}(p)_{\text {tad }}$.

Finally summing up the IR parts of bubble (3.6) and tadpole (3.12) contributions we get:

$$
\begin{aligned}
{\left[S^{\mu \nu}(p)_{\mathrm{bub}}+S^{\mu \nu}(p)_{\mathrm{tad}}\right]_{\mathrm{IR}} } & =\left.\frac{e^{2}}{(4 \pi)^{2}}(\theta p)^{\mu}(\theta p)^{\nu} S_{2}(p)_{\mathrm{bub}}\right|_{\mathrm{IR}} \\
& =-\frac{e^{2}}{(4 \pi)^{2}} \frac{(\theta p)^{\mu}(\theta p)^{\nu}}{(\theta p)^{2}} 8 T_{0}=\frac{e^{2}}{(4 \pi)^{2}}(\theta p)^{\mu}(\theta p)^{\nu} \frac{16}{(\theta p)^{4}}
\end{aligned}
$$

where all IR terms from both diagrams, except the one arising from the bubble, cancels out. Interesting enough is that within this noncommutaive scalar-photon action in the adjont (3.3) we are facing the exact cancelations of all divergences of the higher order terms of noncommutative tensor-parameter $\theta^{\mu \nu}$, showing thus the consistency of our computations.

\subsection{The photon one-loop contribution to scalar 1-PI two point function}

The one-loop adjoint scalar 1-PI two point function in the minimal coupled model consists the tadpole diagram figure 9 and the bubble diagram figure 10. The evaluation is straightforward. We obtain in the end

$$
\Sigma_{(\phi)}(p)_{\operatorname{tad}}=\frac{e^{2}}{(4 \pi)^{2}} 8 T_{0}
$$

and

$$
\begin{aligned}
& \Sigma_{(\phi)}(p)_{\mathrm{bub}}= \\
& -\frac{e^{2}}{(4 \pi)^{2}}\left[\operatorname { t r } \theta \theta \frac { p ^ { 4 } } { ( \theta p ) ^ { 2 } } \left(\left.2(4 \pi)^{-\frac{D}{2}}\left(p^{2}\right)^{\frac{D}{2}-2} \Gamma\left(2-\frac{D}{2}\right) \mathrm{B}\left(\frac{D}{2}-1, \frac{D}{2}-1\right)\right|_{D \rightarrow 4-\epsilon}\right.\right. \\
& \left.-4 I_{K}^{0}-4 I_{H}\right) \\
& +(\theta \theta p)^{2} \frac{p^{4}}{(\theta p)^{4}}\left(\left.4(4 \pi)^{-\frac{D}{2}}\left(p^{2}\right)^{\frac{D}{2}-2} \Gamma\left(2-\frac{D}{2}\right) \mathrm{B}\left(\frac{D}{2}-1, \frac{D}{2}-1\right)\right|_{D \rightarrow 4-\epsilon}\right. \\
& \left.-8 I_{K}^{0}-4 I_{H}\right) \\
& +p^{2}\left(\left.(2 D-4)(4 \pi)^{-\frac{D}{2}}\left(p^{2}\right)^{\frac{D}{2}-2} \Gamma\left(2-\frac{D}{2}\right) \mathrm{B}\left(\frac{D}{2}-1, \frac{D}{2}-1\right)\right|_{D \rightarrow 4-\epsilon}\right. \\
& \left.\left.-8 I_{K}^{0}-12 I_{H}\right)-4 T_{0}\right] .
\end{aligned}
$$




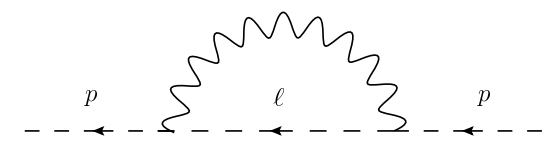

Figure 10. The scalar-photon bubble: $\Sigma_{(\phi)}(p)_{\text {bub }}$.

The total quadratic IR divergence reads

$$
\left[\Sigma_{(\phi)}(p)_{\mathrm{tad}}+\Sigma_{(\phi)}(p)_{\mathrm{bub}}\right]_{\mathrm{IR}}=\frac{e^{2}}{(4 \pi)^{2}} 12 T_{0}=-\frac{e^{2}}{(4 \pi)^{2}} \frac{24}{(\theta p)^{2}}
$$

We will soon see in the next section that this divergence is exactly canceled by the contributions from scalar-photino and scalar self-interaction diagrams.

\section{Noncommutative $\mathcal{N}=2$ SYM U(1) theory and the $\theta$-exact SW map}

The noncommutative $\mathrm{U}(1) \mathcal{N}=2$ super Yang-Mills theory has the following action

$$
\begin{aligned}
S_{\mathcal{N}=2}= & \int-\frac{1}{4} F_{\mu \nu} \star F^{\mu \nu}+\left(\mathcal{D}_{\mu}[A] \Phi\right)^{\dagger} \mathcal{D}^{\mu}[A] \Phi-\frac{e^{2}}{2}\left[\Phi^{\dagger},, \Phi\right]^{2} \\
& +i \bar{\Lambda} \bar{\sigma}^{\mu} \mathcal{D}_{\mu}[A] \Lambda+i \bar{\Psi} \bar{\sigma}^{\mu} \mathcal{D}_{\mu}[A] \Psi+i e \sqrt{2} \Psi\left[\Lambda \stackrel{\star}{\star} \Phi^{\dagger}\right]+i e \sqrt{2} \bar{\Psi}[\bar{\Lambda}, \star, \Phi],
\end{aligned}
$$

in the Wess-Zumino gauge. The noncommutative fields in the previous action constitute the noncommutative $\mathrm{U}(1)$ supermultiplet $\left(A_{\mu}, \Lambda_{\alpha}, \Psi_{\alpha}, \Phi\right) . \Lambda_{\alpha}$ and $\Psi_{\alpha}$ are Weyl fermion fields and $\Phi$ is a complex scalar field. Each field $\Lambda_{\alpha}, \Psi_{\alpha}$ and $\Phi$ transforms under the adjoint action of the $\mathrm{NC} \mathrm{U}(1)$, so that the $\mathrm{NC}$ covariant derivative is $\mathcal{D}_{\mu}[A]=\partial_{\mu}-i\left[A_{\mu} \stackrel{\star}{,}\right]$.

By replacing the noncommutative fields of the action in (4.1) with the $\theta$-exact SeibergWitten maps - see appendix $\mathrm{A}-A_{\mu}\left[a_{\rho} ; \theta\right], \Lambda_{\alpha}\left[a_{\rho}, \lambda_{\alpha} ; \theta\right], \Psi_{\alpha}\left[a_{\rho}, \psi_{\alpha} ; \theta\right], \Phi\left[a_{\rho}, \phi ; \theta\right]$, the action $S_{\mathcal{N}=2}$ is turned into the action of a theory which is an interacting deformation of the free ordinary $\mathrm{U}(1)$ supersymmetric theory for the $\mathrm{U}(1)$ supermultiplet $\left(a_{\mu}, \lambda_{\alpha}, \psi_{\alpha}, \phi\right)$. This deformation is supersymmetric although supersymmetry $-\mathcal{N}=2-$ is nonlinearly realized on the ordinary multiplet $\left(a_{\mu}, \lambda_{\alpha}, \psi_{\alpha}, \phi\right)$; a feature we have already seen in the $\mathcal{N}=1$ SYM case.

The contributions to the action in (4.1) that are needed to compute one-loop 1PI two-point function of each field in $\left(a_{\mu}, \lambda_{\alpha}, \psi_{\alpha}, \phi\right)$ can be readily obtained by using (2.15), (2.16), (3.3) and

$$
\int i e \sqrt{2} \psi\left[\lambda \stackrel{\star}{,} \phi^{\dagger}\right]+i e \sqrt{2} \bar{\psi}[\bar{\lambda} \stackrel{\star}{,} \phi]-\frac{e^{2}}{2}\left(\left[\Phi^{\dagger}, \Phi^{\star}\right]\right)^{2}
$$

The terms in (4.2) yields the scalar-fermion $\mathcal{N}=2$ Feynman rules (E.1) given in appendix E. Now we are ready to display the value of each one-loop Feynman diagram contributing to the two-point functions of the ordinary fields of the theory. 


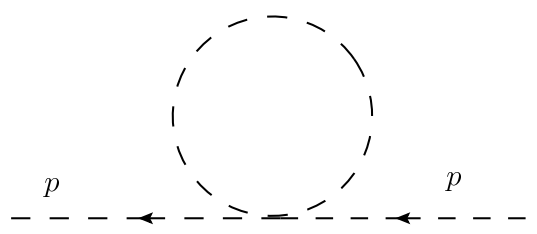

Figure 11. $\mathcal{N}=2$ four-scalar tadpole: $\Sigma_{(\phi)}(p)_{4 \mathrm{sc}-\mathrm{tad}}$.

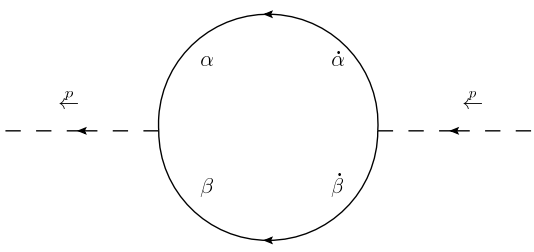

Figure 12. $\mathcal{N}=2$ scalar-photino bubble: $\Sigma_{(\phi)}(p)_{\mathrm{f}-\text { bub }}$.

\subsection{The one-loop 1PI two-point function for photon field $a_{\mu}$}

For $\mathcal{N}=2$ theory one has $n_{f}=n_{s}=2$. Substituting these numbers as well as the scalar bubble and tadpole results to (2.21), and then restricting to quadratic IR divergence only, gives

$$
\left.\Pi^{\mu \nu}(p)\right|_{\mathrm{IR}}=\left.\frac{e^{2}}{(4 \pi)^{2}} \frac{(\theta p)^{\mu}(\theta p)^{\nu}}{(\theta p)^{2}}\left(-16 T_{0}+16 n_{f} T_{0}-8 n_{s} T_{0}\right)\right|_{n_{f}=n_{s}=2}=0,
$$

i.e. clean quadratic IR divergence cancellation. Remaining UV divergences can be expressed using the five-term notation in (2.22) as follows

$$
\begin{aligned}
& \left.\Pi_{1}(p)\right|_{\mathrm{UV}}=\left.\left(\frac{4}{3}-\frac{4}{3} n_{f}-\frac{1}{3} n_{s}+\frac{p^{2}}{(\theta p)^{4}}\left(3 \operatorname{tr} \theta \theta(\theta p)^{2}+4(\theta \theta p)^{2}\right)\right)\left(\frac{2}{\epsilon}+\ln \left(\mu^{2}(\theta p)^{2}\right)\right)\right|_{n_{f}=n_{s}=2}, \\
& \left.\Pi_{2}(p)\right|_{\mathrm{UV}}=\frac{p^{2}}{(\theta p)^{2}}\left(2-\frac{p^{2}(\operatorname{tr} \theta \theta)}{(\theta p)^{2}}\right)\left(\frac{2}{\epsilon}+\ln \left(\mu^{2}(\theta p)^{2}\right)\right) \\
& \left.\Pi_{3}(p)\right|_{\mathrm{UV}}=\frac{p^{2}}{(\theta p)^{2}}\left(\frac{2}{\epsilon}+\ln \left(\mu^{2}(\theta p)^{2}\right)\right) \\
& \left.\Pi_{4}(p)\right|_{\mathrm{UV}}=-4 \frac{p^{4}}{(\theta p)^{4}}\left(\frac{2}{\epsilon}+\ln \left(\mu^{2}(\theta p)^{2}\right)\right) \\
& \left.\Pi_{5}(p)\right|_{\mathrm{UV}}=4 \frac{p^{4}}{(\theta p)^{4}}\left(\frac{2}{\epsilon}+\ln \left(\mu^{2}(\theta p)^{2}\right)\right)
\end{aligned}
$$

\subsection{The one-loop 1PI two-point function for the scalar $\phi$}

The one-loop 1PI two-point function, $\Sigma_{(\phi)}(p)$, of field $\phi$ is the sum of the four diagrams (figure 9, figure 10, figure 11 and figure 12). The first two are already given as equations (3.14) 


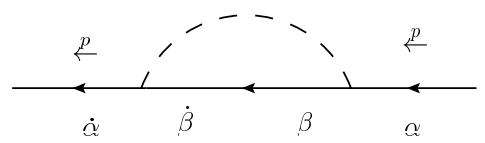

Figure 13. $\mathcal{N}=2$ photino-scalar bubble: $\Sigma^{\dot{\alpha} \alpha}(p)_{\text {scal }}$.

and (3.15) in the last section. The values of the third and fourth diagrams read

$$
\begin{aligned}
& \Sigma_{(\phi)}(p)_{4 \text { sc }- \text { tad }}=-\frac{e^{2}}{(4 \pi)^{2}} 4 T_{0}, \\
& \Sigma_{(\phi)}(p)_{\mathrm{f}-\text { bub }}=8 e^{2} \mu^{4-D} \int \frac{d^{D} \ell}{(2 \pi)^{D}} \frac{p \ell-\ell^{2}}{\ell^{2}(\ell-p)^{2}} \sin ^{2} \frac{\ell \theta p}{2} \\
& =-4 e^{2} \mu^{4-D} \int \frac{d^{D} \ell}{(2 \pi)^{D}} \frac{1}{\ell^{2}} \sin ^{2} \frac{\ell \theta p}{2}\left(2-\frac{p^{2}}{(\ell-p)^{2}}\right) \\
& =\frac{e^{2}}{(4 \pi)^{2}}\left[-8 T_{0}+2 p^{2}\left(\left.\left(4 \pi \mu^{2}\right)^{2-\frac{D}{2}}\left(p^{2}\right)^{\frac{D}{2}-2} \Gamma\left(2-\frac{D}{2}\right) \mathrm{B}\left(\frac{D}{2}-1, \frac{D}{2}-1\right)\right|_{D \rightarrow 4-\epsilon}\right.\right. \\
& \left.\left.\quad-2 I_{K}^{0}\right)\right] .
\end{aligned}
$$

Hence, one gets the following full scalar two-point function:

$$
\Sigma_{(\phi)}(p)=\Sigma_{(\phi)}(p)_{\text {tad }}+\Sigma_{(\phi)}(p)_{\text {bub }}+\Sigma_{(\phi)}(p)_{4 s c-\text { tad }}+\Sigma_{(\phi)}(p)_{\mathrm{f}-\text { bub }},
$$

which is again quadratic IR divergence free, as

$$
\begin{aligned}
\left.\Sigma_{(\phi)}(p)\right|_{\mathrm{IR}} & =\left.\left(\Sigma_{(\phi)}(p)_{\mathrm{tad}}+\Sigma_{(\phi)}(p)_{\mathrm{bub}}+\Sigma_{(\phi)}(p)_{4 \mathrm{sc}-\mathrm{tad}}+\Sigma_{(\phi)}(p)_{\mathrm{f}-\mathrm{bub}}\right)\right|_{\mathrm{IR}} \\
& =\frac{e^{2}}{(4 \pi)^{2}}\left(12 T_{0}-8 T_{0}-4 T_{0}\right)=0 .
\end{aligned}
$$

The UV divergence reads

$$
\begin{aligned}
\left.\Sigma_{(\phi)}(p)\right|_{\mathrm{UV}} & =\left.\left(\Sigma_{(\phi)}(p)_{\mathrm{bub}}+\Sigma_{(\phi)}(p)_{\mathrm{f}-\mathrm{bub}}\right)\right|_{\mathrm{UV}} \\
& =-2 \frac{e^{2}}{(4 \pi)^{2}} p^{2}\left(1+\operatorname{tr} \theta \theta \frac{p^{2}}{(\theta p)^{2}}+2(\theta \theta p)^{2} \frac{p^{2}}{(\theta p)^{4}}\right)\left(\frac{2}{\epsilon}+\ln \left(\mu^{2}(\theta p)^{2}\right)\right) .
\end{aligned}
$$

4.3 The one-loop 1PI two-point function for photinos $\lambda_{\alpha}$ and $\psi_{\alpha}$

In the $\mathcal{N}=2$ theory there is a scalar-photino loop (figure 13) alongside the photon-photino loop contribution which is identical to the $\mathcal{N}=1$ theory value (2.46) for each of the two photinos.

The scalar-photino loop integral gives the following contribution

$$
\begin{aligned}
\Sigma^{\dot{\alpha} \alpha}(p)_{\mathrm{scal}}= & 8 e^{2} \mu^{4-D} \int \frac{d^{D} \ell}{(4 \pi)^{D}} \sin ^{2} \frac{\ell \theta p}{2} \frac{\ell^{\mu}-p^{\mu}}{\ell^{2}(\ell-p)^{2}} \bar{\sigma}_{\mu}^{\dot{\alpha} \alpha} \\
= & -\frac{e^{2}}{(4 \pi)^{2}} p^{\mu} \bar{\sigma}_{\mu}^{\dot{\alpha} \alpha} 2\left(\left.\left(4 \pi \mu^{2}\right)^{2-\frac{D}{2}}\left(p^{2}\right)^{\frac{D}{2}-2} \Gamma\left(2-\frac{D}{2}\right) \mathrm{B}\left(\frac{D}{2}-1, \frac{D}{2}-1\right)\right|_{D \rightarrow 4-\epsilon}\right. \\
& \left.-2 I_{K}^{0}\right) .
\end{aligned}
$$


Total photino two-point function is finally given as a sum:

$$
\Sigma_{(\lambda, \psi)}^{\dot{\alpha} \alpha}(p)=\Sigma^{\dot{\alpha} \alpha}(p)_{\mathrm{tad}}+\Sigma^{\dot{\alpha} \alpha}(p)_{\mathrm{bub}}+\Sigma^{\dot{\alpha} \alpha}(p)_{\mathrm{scal}}
$$

It is quadratic IR divergence free and it has the following UV divergence

$$
\left.\Sigma_{(\lambda, \psi)}^{\dot{\alpha} \alpha}(p)\right|_{\mathrm{UV}}=-\frac{e^{2}}{(4 \pi)^{2}} \sigma_{\mu}^{\dot{\alpha} \alpha} p^{\mu}\left(2+\operatorname{tr} \theta \theta \frac{p^{2}}{(\theta p)^{2}}+2(\theta \theta p)^{2} \frac{p^{2}}{(\theta p)^{4}}\right)\left(\frac{2}{\epsilon}+\ln \left(\mu^{2}(\theta p)^{2}\right)\right) .
$$

\section{Noncommutative $\mathcal{N}=4$ SYM U(1) theory and the $\theta$-exact SW map}

Let $\left(A_{\mu}, \Lambda_{\alpha i}, \Phi_{m}\right), i=1, \ldots, 4, m=1, \ldots, 6$ define the noncommutative $\mathrm{U}(1) \mathcal{N}=4$ supermultiplet; then, the action of the noncommutative $\mathrm{U}(1) \mathcal{N}=4$ super Yang-Mills theory reads

$$
\begin{aligned}
S_{\mathcal{N}=4}= & \int-\frac{1}{4} F_{\mu \nu} \star F^{\mu \nu}+i \bar{\Lambda}^{i} \bar{\sigma}^{\mu} \mathcal{D}_{\mu}[A] \Lambda_{i}+\frac{1}{2} \mathcal{D}_{\mu}[A] \Phi_{m} \mathcal{D}^{\mu}[A] \Phi_{m}+\left(\frac{e}{2}\left[\Phi_{m} \star \Phi_{n}\right]\right)^{2} \\
& +i \frac{e}{2}\left(\tilde{\sigma}^{-1}\right)^{i j} \Lambda_{i}\left[\Lambda_{j}, \Phi_{m}\right]-i \frac{e}{2}(\tilde{\sigma})_{i j} \bar{\Lambda}^{i}\left[\bar{\Lambda}^{j}, \Phi_{m}\right]
\end{aligned}
$$

The matrices $4 \times 4,(\tilde{\sigma})_{i j}$ and $\left(\tilde{\sigma}^{-1}\right)^{i j}$ give rise to the IRREP of the Dirac matrices in 8 Euclidean dimensions; further details can be found in [22]. Let us recall that $A_{\mu}$ is the noncomutative gauge field, that $\Lambda_{\alpha i}$ is a noncommutative Weyl field and that $\Phi_{m}$ is a noncommutative real scalar field. The noncommutative $\mathrm{U}(1)$ acts by the adjoint action on $\Lambda_{\alpha i}$ and $\Phi_{m}$, and hence $\mathcal{D}_{\mu}[A]=\partial_{\mu}-i\left[A_{\mu} \stackrel{\star}{,}\right]$.

By replacing, in $S_{\mathcal{N}=4}$ above, the fields $A_{\mu}, \Lambda_{\alpha i}$ and $\Phi_{m}$ with the corresponding $\theta$-exact Seiberg-Witten maps - namely, $A_{\mu}\left[a_{\rho} ; \theta\right], \Lambda_{\alpha i}\left[a_{\rho}, \lambda_{\alpha} ; \theta\right]$ and $\Phi_{m}\left[a_{\rho}, \phi ; \theta\right]$, respectively, we obtain an action which defines an interacting deformation of the ordinary $\mathcal{N}=4$ SYM theory in the Wess-Zumino gauge. This deformed action is expressed in terms of the fields of the ordinary $\mathcal{N}=4$ Yang-Mills supermultiplet $\left(a_{\mu}, \lambda_{\alpha i}, \phi_{m}\right), i=1, \ldots, 4, m=$ $1, \ldots, 6$ and it is invariant (on-shell) under the deformed supersymmetric transformations of the ordinary supermultiplet $\left(a_{\mu}, \lambda_{\alpha i}, \phi_{m}\right)$ which give rise to the $\mathcal{N}=4$ supersymmetric transformations of the fields in $\left(A_{\mu}, \Lambda_{\alpha i}, \Phi_{m}\right)$. As in the $\mathcal{N}=1$ and $\mathcal{N}=2$ cases, the supersymmetry transformations of the ordinary fields that leave $S_{\mathcal{N}=4}$ in (5.1) invariant gives rise to on-shell nonlinear realization of $\mathcal{N}=4$ supersymmetry algebra.

The contributions to the action in (5.1) that are needed to compute one-loop 1PI two-point function of each field in $\left(a_{\mu}, \lambda_{\alpha}, \psi_{\alpha}, \phi\right)$ can easily be obtained by using (2.18), (2.19), (3.3) and

$$
\int\left(\frac{e}{2}\left[\phi_{m} \stackrel{\star}{,} \phi_{n}\right]\right)^{2}+\frac{i e}{2}\left(\tilde{\sigma}^{-1}\right)^{i j} \lambda_{i}\left[\lambda_{j} \stackrel{\star}{,} \phi_{m}\right]-\frac{i e}{2}(\tilde{\sigma})_{i j} \bar{\lambda}^{i}\left[\bar{\lambda}^{j} \stackrel{\star}{,} \phi_{m}\right] .
$$

The terms in (5.2) yields the Feynman rules given in appendix D.

Below we shall display the value of each one-loop Feynman diagram contributing to the two-point functions of the ordinary fields of the theory. 


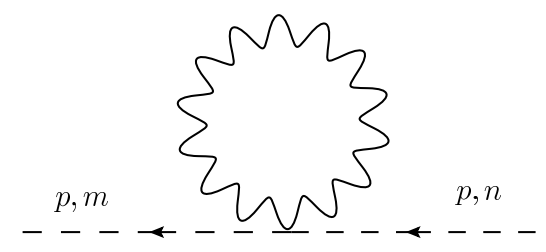

Figure 14. $\mathcal{N}=4$ scalar-photon tadpole: $\Sigma_{m n}(p)_{\text {tad }}$.

\subsection{The one-loop 1PI two-point function for massless vector field $a_{\mu}$}

The $\mathcal{N}=4 a_{\mu}$ 1PI two-point function follows the general formula (2.21), only with $n_{f}=$ 4 and $n_{s}=6$. One can immediately recognize clean cancelation of the quadratic IR divergences after substituting $\mathcal{N}=4 \rightarrow n_{f}=4, n_{s}=6$ into (4.3).

\subsection{The one-loop 1PI two-point function for the scalar $\phi_{m}$}

The one-loop 1PI two-point function, $\Sigma_{m n}(p)$, of the field $\phi_{m}$ is the sum of five diagrams figures 14, 15, 16 and 17, whose values read

$$
\begin{aligned}
& \Sigma_{m n}(p)_{4 \mathrm{sc}-\mathrm{tad}}=\frac{e^{2}}{(4 \pi)^{2}} 20 \delta_{m n} T_{0} \\
& \Sigma_{m n}(p)_{\mathrm{f}-\mathrm{bub}}=32 e^{2} \mu^{4-D} \delta_{m n} \int \frac{d^{D} \ell}{(2 \pi)^{D}} \frac{p \ell-\ell^{2}}{\ell^{2}(\ell-p)^{2}} \sin ^{2} \frac{\ell \theta p}{2} \\
& =\frac{8 e^{2}}{\left(4 \pi \mu^{2}\right)^{2}} \delta_{m n}\left[-4 T_{0}+p^{2}\left(\left.(4 \pi)^{2-\frac{D}{2}}\left(p^{2}\right)^{\frac{D}{2}-2} \Gamma\left(2-\frac{D}{2}\right) \mathrm{B}\left(\frac{D}{2}-1, \frac{D}{2}-1\right)\right|_{D \rightarrow 4-\epsilon}\right.\right. \\
& \left.\left.\quad-2 I_{K}^{0}\right)\right]
\end{aligned}
$$

hence

$$
\Sigma_{m n}(p)=\Sigma_{m n}(p)_{\mathrm{tad}}+\Sigma_{m n}(p)_{\mathrm{bub}}+\Sigma_{m n}(p)_{4 \mathrm{sc}-\mathrm{tad}}+\Sigma_{m n}(p)_{\mathrm{f}-\mathrm{bub}},
$$

is again IR divergence free, only this time we have

$$
\begin{aligned}
\left.\Sigma_{m n}(p)\right|_{\mathrm{IR}} & =\left.\left(\Sigma_{m n}(p)_{\mathrm{tad}}+\Sigma_{m n}(p)_{\mathrm{bub}}+\Sigma_{m n}(p)_{4 \mathrm{sc}-\mathrm{tad}}+\Sigma_{m n}(p)_{\mathrm{f}-\mathrm{bub}}\right)\right|_{\mathrm{IR}} \\
& =\frac{e^{2}}{(4 \pi)^{2}} \delta_{m n}\left(12 T_{0}-32 T_{0}+20 T_{0}\right)=0 .
\end{aligned}
$$

The UV part reads

$$
\left.\Sigma_{m n}(p)\right|_{\mathrm{UV}}=\frac{e^{2}}{(4 \pi)^{2}} \delta_{m n} p^{2}\left(4-2 \operatorname{tr} \theta \theta \frac{p^{2}}{(\theta p)^{2}}-4(\theta \theta p)^{2} \frac{p^{2}}{(\theta p)^{4}}\right)\left(\frac{2}{\epsilon}+\ln \left(\mu^{2}(\theta p)^{2}\right)\right) .
$$

\subsection{The one-loop 1PI two-point function for $\lambda_{\alpha i}$}

The one-loop 1PI two-point function, $\Sigma_{j}^{\dot{\alpha} \alpha}{ }_{j}(p)$, of the field $\lambda_{\alpha i}$ is the sum of the three diagrams figure 18, figure 19 and figure 20 whose values read

$$
\begin{aligned}
\sum_{j}^{\dot{\alpha} \alpha i}(p)_{\mathrm{tad}} & =0, \\
\sum_{j}^{\dot{\alpha} \alpha i}(p)_{\mathrm{bub}} & =-\frac{e^{2}}{(4 \pi)^{2}} \bar{\sigma}_{\mu}^{\dot{\alpha} \alpha} \delta_{j}^{i}\left[p^{\mu} N_{1}(p)+(\theta \theta p)^{\mu} N_{2}(p)\right],
\end{aligned}
$$




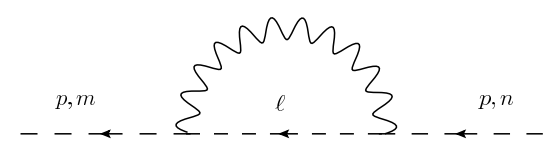

Figure 15. $\mathcal{N}=4$ scalar-photon bubble: $\Sigma_{m n}(p)_{\text {bub }}$.

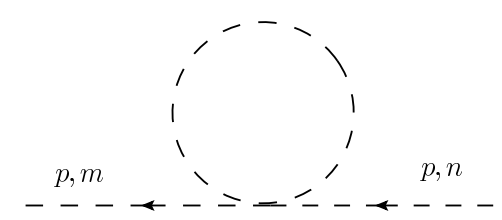

Figure 16. $\mathcal{N}=4$ four-scalar tadpole: $\Sigma_{m n}(p)_{4 \mathrm{sc}-\text { tad }}$.
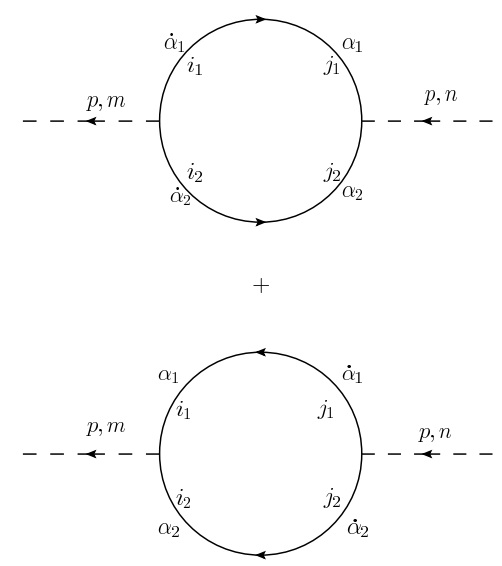

Figure 17. $\mathcal{N}=4$ scalar-photino bubbles: $\Sigma_{m n}(p)_{\text {f-bub }}$.

with $N_{1,2}(p)$ being given in (2.47) and (2.48), respectively, and

$$
\begin{aligned}
\sum_{j}^{\dot{\alpha} \alpha i}(p)_{\mathrm{scal}}= & 24 e^{2} \mu^{4-D} \delta_{j}^{i} \int \frac{d^{D} \ell}{(4 \pi)^{D}} \sin ^{2} \frac{\ell \theta p}{2} \frac{\ell^{\mu}-p^{\mu}}{\ell^{2}(\ell-p)^{2}} \bar{\sigma}_{\mu}^{\dot{\alpha} \alpha} \\
= & -\frac{6 e^{2}}{(4 \pi)^{2}} \delta_{j}^{i} p^{\mu} \sigma_{\mu}^{\dot{\alpha} \alpha}\left(\left.\left(4 \pi \mu^{2}\right)^{2-\frac{D}{2}}\left(p^{2}\right)^{\frac{D}{2}-2} \Gamma\left(2-\frac{D}{2}\right) \mathrm{B}\left(\frac{D}{2}-1, \frac{D}{2}-1\right)\right|_{D \rightarrow 4-\epsilon}\right. \\
& \left.-2 I_{K}^{0}\right)
\end{aligned}
$$

Hence,

$$
\Sigma_{j}^{\dot{\alpha} \alpha i}(p)=\Sigma_{j}^{\dot{\alpha} \alpha i}(p)_{\mathrm{tad}}+\Sigma_{j}^{\dot{\alpha} \alpha i}(p)_{\mathrm{bub}}+\Sigma_{j}^{\dot{\alpha} \alpha i}(p)_{\mathrm{scal}},
$$

is quadratic IR divergence free, and the total UV divergences is presented below

$$
\left.\sum_{j}^{\dot{\alpha} \alpha i}(p)\right|_{\mathrm{UV}}=-\frac{e^{2}}{(4 \pi)^{2}} \delta_{j}^{i} \sigma_{\mu}^{\dot{\alpha} \alpha} p^{\mu}\left(6+\operatorname{tr} \theta \theta \frac{p^{2}}{(\theta p)^{2}}+2(\theta \theta p)^{2} \frac{p^{2}}{(\theta p)^{4}}\right)\left(\frac{2}{\epsilon}+\ln \left(\mu^{2}(\theta p)^{2}\right)\right) .
$$




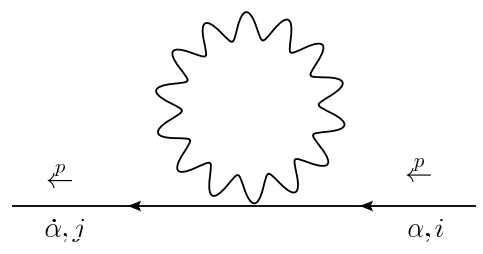

Figure 18. $\mathcal{N}=4$ photino-photon tadpole: $\sum_{j}^{\dot{\alpha} \alpha} \underset{j}{i}(p)_{\operatorname{tad}}$.

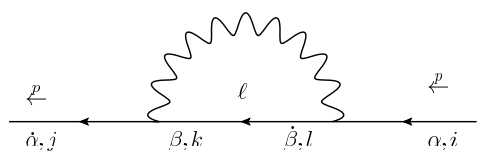

Figure 19. $\mathcal{N}=4$ photino-photon bubble: $\sum_{j}^{\dot{\alpha} \alpha}{ }_{j}^{i}(p)_{\text {bub }}$.

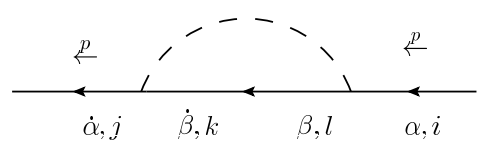

Figure 20. $\mathcal{N}=4$ photino-scalar bubble: $\sum_{j}^{\dot{\alpha} \alpha}(p)_{\text {scal }}$.

\section{Effect of gauge fixing on photon two point function}

In the prior sections we have shown that the quadratic IR divergent contribution to the photon two point function can be canceled by introducing supersymmetry. Yet we have still two unanswered question: first, all of our computations above are carried out in the commutative Feynman gauge, which, albeit convenient, is just one specific choice. We do not know whether the cancelation we found would be changed by a change of gauge fixing. Second, we have quite complicated UV divergence in the Feynman gauge in general, which may be modified by changing gauge fixing, as in the commutative gauge theories. To study these two issues we introduce in this section a new, non-local and nonlinear gauge fixing based on the Seiberg-Witten map then evaluate its effect to the photon two point function.

\subsection{The noncommutative Feynman gauge fixing action}

We introduce a new gauge fixing for non-local U(1) gauge theory via the $\theta$-exact SeibergWitten map. In terms of BRST language, this gauge fixing contains BRST-auxiliary field $B$, and it is given by

$$
\begin{aligned}
S & =S_{\mathrm{U}(1)}+S_{\mathrm{gf}}=S_{\mathrm{U}(1)}+s \int \bar{\omega}\left(\partial_{\mu} A^{\mu}\left(a_{\mu}, \theta^{i j}\right)+\frac{B}{2}\right), \\
S_{\mathrm{gf}} & =\int B\left(\partial_{\mu} A^{\mu}+\frac{B}{2}\right)-\bar{\omega} s\left(\partial_{\mu} A^{\mu}\right)=\int \frac{1}{2}\left(B+\partial_{\mu} A^{\mu}\right)^{2}-\frac{1}{2}\left(\partial_{\mu} A^{\mu}\right)^{2}-\bar{\omega} \partial_{\mu}\left(s A^{\mu}\right),
\end{aligned}
$$

with $s$ being regular U(1) BRST transformations $s a_{\mu}=\partial_{\mu} \omega$, where $\omega$ is the $\mathrm{U}(1)$ ghost. Next we use consistency condition for SW map to get $s A_{\mu}\left(a_{\mu}, \theta^{i j}\right)=D_{\mu} \Omega$, where $\Omega$ is $\mathrm{U}_{\star}(1)$ ghost and $D_{\mu}$ the $\mathrm{U}_{\star}(1)$ covariant derivative in the adjoint representation $D_{\mu}=$ $\partial_{\mu}+i\left[A_{\mu} \stackrel{\star}{,}\right]$. 


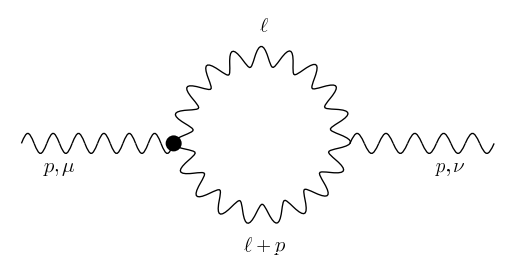

Figure 21. Gauge fixing L: 3-photon bubble.

Since the SW map for $\Omega$ is actually the same as for the NC gauge parameter $\Lambda$ from [15] we can derive the photon-photon and photon-ghost coupling in this gauge. So by using the following BRST transformations

$$
\begin{aligned}
s \bar{\omega} & =B, \quad s B=0, \\
s A^{\mu}\left(a_{\mu}, \theta^{i j}\right) & =s_{\mathrm{NC}} A^{\mu}=D^{\mu} \Omega,
\end{aligned}
$$

from (6.1) is produced the following gauge fixing action

$$
S_{\mathrm{gf}}=\int \frac{1}{2}\left(B+\partial_{\mu} A^{\mu}\right)^{2}-\frac{1}{2}\left(\partial_{\mu} A^{\mu}\right)^{2}-\bar{\omega} \partial_{\mu} D^{\mu} \Omega
$$

which after the application of the SW map resulting Feynman rules for the gauge fixing and ghost induced diagrams given in appendix F. We name this gauge as "noncommutative (NC) Feynman gauge" as it is formally identical to the Feynman gauge in a $U_{\star}(1)$ gauge theory.

\subsection{One-loop contributions from the new NC gauge fixing action}

The new gauge fixing action (6.3) introduces additional terms to the three and four photon self-couplings as well as photon-ghost couplings, as summarized in (F.1). Unlike the three and four photon couplings in the commutative Feynman gauge $[16,18]$, these new interaction terms are no longer transverse. It then becomes intriguing how the sum of the resulting loop integrals behave.

From Feynman rules (F.1) we find the following diagrams figures 21-26 contributing to the one loop photon two point function. Denoting the total sum of figures 21 to 26 as $\Pi_{\mathrm{gf}_{\text {total }}}^{\mu \nu}$, it turns out to be convenient to split it into two partial sums

$$
\Pi_{\mathrm{gf}_{\text {total }}}^{\mu \nu}=\Pi_{\mathrm{gf}_{\text {mix }}}^{\mu \nu}+\Pi_{\mathrm{gf}}^{\mu \nu}
$$

Here $\Pi_{\text {gf }_{\text {mix }}}^{\mu \nu}$ presents the sum over figures 21 and 22, which contain one 3-photon vertex from the classical action and the other from gauge fixing action, while $\Pi_{\mathrm{gf}}^{\mu \nu}$ sums over the rest of them which are solely from the gauge fixing. ${ }^{2}$

The evaluation of diagrams in figure 21-26 follows substantially the standard procedure used in the prior section, except the rising of the two new types of tadpole integrals.

\footnotetext{
${ }^{2}$ One more reason is that gauge fixing (6.1) and/or (6.3) can be added to any $\mathrm{U}(1)$ gauge invariant action, particularly the free $\mathrm{U}(1)$ action $S_{\text {free }}=-\frac{1}{4} \int f_{\mu \nu} f^{\mu \nu}$. In this case $\Pi_{\mathrm{gf}}^{\mu \nu}$ would present the whole contribution to the one loop 1-PI photon two point function. Thus it is convenient to isolate it out.
} 


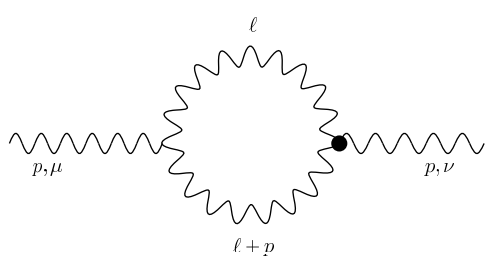

Figure 22. Gauge fixing R: 3-photon bubble.

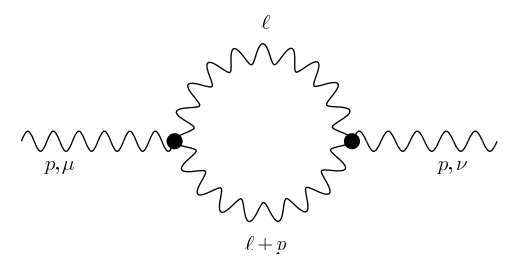

Figure 23. Gauge fixing L\&R: 3-photon bubble.

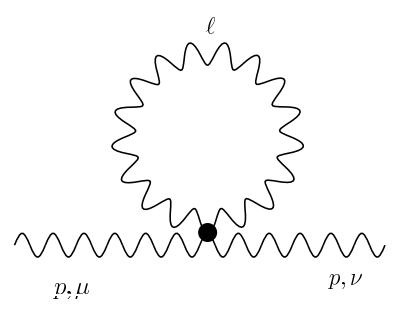

Figure 24. Gauge fixing T: 4-photon tadpole.

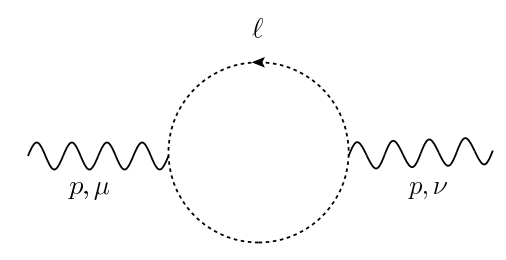

Figure 25. Photon-ghost bubble.

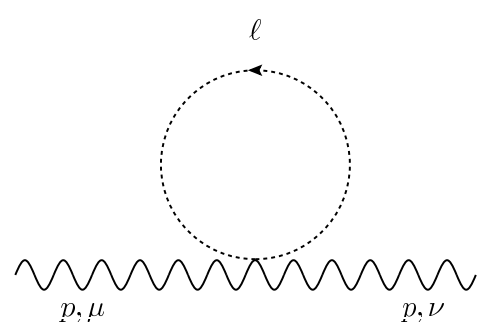

Figure 26. 2Photons-ghost tadpole. 
The first one takes the form of the second term in (2.44) so it can be removed by our regularization prescription. The second one is a tadpole integral without any loop momenta in the numerator i.e. $\int \frac{d^{D} k}{(2 \pi)^{D}} k^{-2} f_{\star_{2}}(k, p)^{2}$. This integral contains total effective loop momenta power $\ell^{-4}$ because of the additional denominator $(k \theta p)^{-2}$ from the nonlocal factor $f_{\star_{2}}(k, p)^{2}$, which is below the minimal power for the commutative tadpole integral to vanish [23]. Consequently we observe unregularized UV divergence when computing this integral by transforming it into the bubble or applying the n-nested zero regulator method [23]. We develop an alternative prescription (B.21) based on the parametrization (2.40) which is capable of dimensionally-regularizing this integral into a $1 / \epsilon$ divergence plus the logarithmic UV/IR mixing term $\ln \left(\mu^{2}(\theta p)^{2}\right)$ at the $D \rightarrow 4-\epsilon \operatorname{limit}$ (B.22).

We are able to express both $\Pi_{\mathrm{gf} \text { mix }}^{\mu \nu}$ and $\Pi_{\mathrm{gf}}^{\mu \nu}$ appropriately once $T_{-2}$ is added to the prior basis integral set $T_{0}, I_{K}^{0}, I_{K}^{1}$ and $I_{H}$. The outcome is listed as below

$$
\begin{aligned}
\Pi_{\mathrm{gf}_{\text {mix }} \nu=} & \frac{e^{2}}{(4 \pi)^{2}}\left(2 p^{\mu} p^{\nu}+p^{\{\mu}(\theta \theta p)^{\nu\}} \frac{p^{2}}{(\theta p)^{2}}\right) \cdot\left(4+4 I_{H}\right) \\
\Pi_{\mathrm{gf}}^{\mu \nu}= & \frac{e^{2}}{(4 \pi)^{2}}\left(p^{\mu} p^{\nu} \cdot \Pi_{A}+p^{\{\mu}(\theta \theta p)^{\nu\}} \Pi_{B}\right) \\
\Pi_{A}= & -\frac{1}{2}\left[\left.\left(4 \pi \mu^{2}\right)^{2-\frac{D}{2}}\left(p^{2}\right)^{\frac{D}{2}-2} \cdot 4 \cdot \Gamma\left(2-\frac{D}{2}\right) \mathrm{B}\left(\frac{D}{2}-1, \frac{D}{2}-1\right)\right|_{D \rightarrow 4-\epsilon}\right. \\
& \cdot\left(1-\frac{p^{2}}{(\theta p)^{2}}\left(\operatorname{tr} \theta \theta(\theta p)^{2}+(\theta \theta p)^{2}\right)\right)+\frac{1}{2} p^{2} \operatorname{tr} \theta \theta T_{-2}-8 I_{K}^{0} \\
& \left.+2 \frac{p^{2}}{(\theta p)^{2}}\left(\operatorname{tr} \theta \theta(\theta p)^{2}\left(4 I_{K}^{0}+4 I_{H}\right)+(\theta \theta p)^{2}\left(8 I_{K}^{0}+4 I_{H}\right)\right)\right] \\
\Pi_{B}= & -\frac{1}{2}\left(\left.\left(4 \pi \mu^{2}\right)^{2-\frac{D}{2}}\left(p^{2}\right)^{\frac{D}{2}-2} \cdot 2(D-1) \cdot \Gamma\left(2-\frac{D}{2}\right) \mathrm{B}\left(\frac{D}{2}-1, \frac{D}{2}-1\right)\right|_{D \rightarrow 4-\epsilon}\right. \\
& \left.-\frac{1}{4} p^{2} T_{-2}-\frac{p^{2}}{(\theta p)^{2}} \cdot 3 \cdot\left(4 I_{K}^{0}+4 I_{H}\right)\right)
\end{aligned}
$$

One can immediately notice that $\Pi_{\mathrm{gf}_{\text {total }}}^{\mu \nu}$ contains only two tensor structures $p^{\mu} p^{\nu}$ and $p^{\{\mu}(\theta \theta p)^{\nu\}}$ which can not be combined into a transverse sum. The loss of transversality appears to be, of course, surprising. However we are going to develop reasoning/arguments for this seeming odd behavior in the next section and show that it is in fact understandable.

\section{Gauge fixing contribution without integrating out BRST-auxiliary field}

In order to achieve simple transversality we conclude that one has to keep BRST auxiliary field $B$ from being integrated out. Arguments for that are as follows.

Starting with the action (6.1) we write a generating functional

$$
\begin{aligned}
Z\left[J^{\mu}, j, \bar{j}, h\right] & =\int D a_{\mu} D \omega D \bar{\omega} D B \cdot \exp \left[i\left(S+\int\left(J^{\mu} a_{\mu}+\bar{j} \omega+j \bar{\omega}+h B\right)\right)\right] \\
& =\exp \left[i W\left[J^{\mu}, j, \bar{j}, h\right]\right]
\end{aligned}
$$


from where we have effective action in terms of "currents":

$$
\Gamma\left[J^{\mu}, j, \bar{j}, h\right]=W\left[J^{\mu}, j, \bar{j}, h\right]+\int\left(J^{\mu} a_{\mu}+\bar{j} \omega+j \bar{\omega}+h B\right) .
$$

Regular BRST transformation $s$ acting on $Z$ vanish, thus we have:

$$
\begin{aligned}
s Z=0 & =i \int D a_{\mu} D \omega D \bar{\omega} D B\left(J^{\mu} \partial_{\mu} \omega-j \cdot B\right) \cdot \exp \left[i\left(S+\int\left(J^{\mu} a_{\mu}+\bar{j} \omega+j \bar{\omega}+h B\right)\right)\right] \\
& \Longrightarrow \int\left(\frac{\delta \Gamma}{\delta a_{\mu}} \partial_{\mu} \omega+B \frac{\delta \Gamma}{\delta \bar{\omega}}\right)=\int\left(-\omega \partial_{\mu} \frac{\delta \Gamma}{\delta a_{\mu}}+B \frac{\delta \Gamma}{\delta \bar{\omega}}\right)=0 .
\end{aligned}
$$

Since the transversality condition means $\partial_{\mu} \frac{\delta \Gamma}{\delta a_{\mu}}=0$, which is satisfied in equation (7.3) only for $B=0$. This however is not allowed if we integrate out the $B$ field. Thus we do not perform that, instead we construct a propagator from the following doublet combina$\operatorname{tion}\left(\begin{array}{c}a_{\mu} \\ B\end{array}\right)$.

\subsection{Formal analysis}

In order to compute the two point function(s) within the presence of the B-field, we must define the propagator(s) for the "kind of strange" vector-scalar field $\left(\begin{array}{c}a_{\mu} \\ B\end{array}\right)$ doublet. Starting with

$$
S_{\mathrm{U}(1)}=\int-\frac{1}{4} f_{\mu \nu} f^{\mu \nu}=\frac{1}{2} \int a_{\mu}\left(\partial^{2} g^{\mu \nu}-\partial^{\mu} \partial^{\nu}\right) a_{\nu},
$$

we get a quadratic part of $S$

$$
S_{\text {quadratic }}=\frac{1}{2} \int\left(a_{\mu}\left(\partial^{2} g^{\mu \nu}-\partial^{\mu} \partial^{\nu}\right) a_{\nu}+2 B \cdot \partial_{\mu} a^{\mu}+B^{2}\right),
$$

whose Fourier transform is as follows

$$
\begin{aligned}
\tilde{S}_{\text {quadratic }}= & \frac{1}{2} \int \frac{d^{4} k}{(2 \pi)^{4}}\left(\tilde{a}^{\mu}(-k)\left(-k^{2} g_{\mu \nu}+k_{\mu} k_{\nu}\right) \tilde{a}^{\nu}(k)\right. \\
& \left.+i \tilde{B}(-k) k_{\mu} \tilde{a}^{\mu}(k)-i k_{\mu} \tilde{a}^{\mu}(-k) \tilde{B}(k)+\tilde{B}(-k) \tilde{B}(k)\right) \\
= & \frac{1}{2} \int \frac{d^{4} k}{(2 \pi)^{4}}\left(\tilde{a}_{\mu}(-k), \tilde{B}(-k)\right) T_{0}\left(\begin{array}{c}
\tilde{a}_{\nu}(k) \\
\tilde{B}(k)
\end{array}\right) .
\end{aligned}
$$

The Hermitian matrix

$$
T_{0}=\left(\begin{array}{cc}
T_{0_{11}}^{\mu \nu} & T_{0_{12}}^{\mu} \\
T_{0_{21}}^{\nu} & T_{0_{22}}
\end{array}\right)=\left(\begin{array}{cc}
-k^{2} g^{\mu \nu}+k^{\mu} k^{\nu} & -i k^{\mu} \\
i k^{\nu} & 1
\end{array}\right),
$$

is then the inverse of the propagator in the momentum space. Next we inverse $T_{0}$ to obtain

$$
T_{0}^{-1}=\left(\begin{array}{cc}
G_{\rho \mu} & A_{\rho} \\
B_{\mu} & G
\end{array}\right) \Longrightarrow T^{-1} \cdot T_{0}=1=\left(\begin{array}{cc}
\delta_{\rho}^{\nu} & 0 \\
0 & 1
\end{array}\right) .
$$



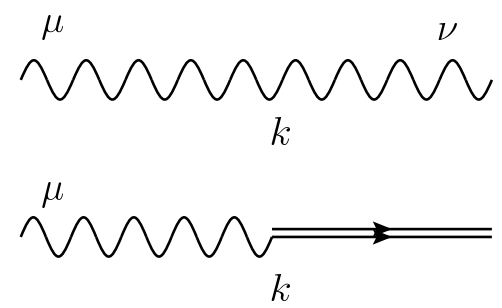

$-\frac{k^{\mu}}{k^{2}}$

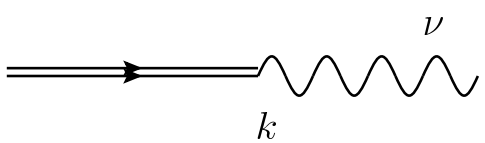

$\frac{k^{\nu}}{k^{2}}$

Figure 27. Illustration of the above procedure in computing photon propagator. BRST-auxiliary field $B$ (Bauxiliary) is denoted by a double full line.

From (7.7) and (7.8) we have four equations:

$$
\begin{aligned}
\delta_{\rho}^{\nu} & =-k^{2} G_{\rho \nu}+k^{\mu} G_{\rho \mu} k^{\nu}+i A_{\rho} k^{\nu}, & & =-i k^{\mu} G_{\rho \mu}+A_{\rho} \\
0 & =-k^{2} B^{\nu}+k^{\mu} B_{\mu} k^{\nu}+i G k^{\nu}, & 1 & =-i B_{\mu} k^{\mu}+1,
\end{aligned}
$$

which we solve by using simple Ansatz: $A_{\rho}=A \cdot k_{\rho}, B_{\mu}=B \cdot k_{\mu} \Longrightarrow G=0, B=$ $\frac{i}{k^{2}} \Longrightarrow A=B^{\dagger}=\frac{-i}{k^{2}} \Longrightarrow G_{\rho \mu}=-\frac{g_{\rho \mu}}{k^{2}}$. Taking into account a text book convention $\left(-k^{2} g^{\mu \nu}+k^{\mu} k^{\nu}\right) G_{\mu \nu}=i \delta_{\rho}^{\mu}$ for the phase factor we add overall factor $i$ and obtained correct photon propagator, as illustrated in figure 27.

One can write down the usual field redefinition $B^{\prime}=B+\partial_{\mu} a^{\mu}$ in (6.3) in the Fourier transformed context as

$$
\left(\begin{array}{c}
\tilde{a}_{\mu}(k) \\
\tilde{B}^{\prime}(k)
\end{array}\right)=A_{0} \cdot\left(\begin{array}{c}
\tilde{a}_{\mu}(k) \\
\tilde{B}(k)
\end{array}\right)=\left(\begin{array}{cc}
1 & 0 \\
i k^{\mu} & 1
\end{array}\right) \cdot\left(\begin{array}{c}
\tilde{a}_{\mu}(k) \\
\tilde{B}(k)
\end{array}\right) .
$$

The $A_{0}^{-1}$ then diagonalizes the bilinear form (7.7) into

$$
T_{0}^{\prime}=A_{0}^{-1^{\dagger}} T_{0} A_{0}^{-1}=\left(\begin{array}{cc}
-k^{2} g^{\mu \nu} & 0 \\
0 & 1
\end{array}\right)
$$

It is easy to see that inverting this $T_{0}^{\prime}$ gives the expected Feynman propagator. From that viewpoint the B-integration can be achieved by diagonalization. One can formally generalize the tree level diagonalization procedure to the one loop. Consider in general the one loop corrections as another Hermitian matrix $T_{1}$ adding to the $T_{0}$ matrix, we write the quadratic part of the 1-loop corrected effective action in the momentum space as

$$
\tilde{\Gamma}_{\text {quadratic }}^{1}=\frac{1}{2} \int \frac{d^{4} k}{(2 \pi)^{4}}\left(\tilde{a}_{\mu}(-k), \tilde{B}(-k)\right)\left(T_{0}+T_{1}\right)\left(\begin{array}{c}
\tilde{a}_{\nu}(k) \\
\tilde{B}(k)
\end{array}\right) .
$$


Next we express $T_{1}$ in terms of its components

$$
T_{1}=\left(\begin{array}{cc}
T_{1_{11}}^{\mu \nu} & i T_{1_{12}}^{\mu}=-i T_{1_{21}}^{\mu} \\
i T_{1_{21}}^{\nu} & T_{1_{22}}
\end{array}\right)
$$

The Slavnov-Taylor identity (7.3) then requires that $T_{1_{11}}^{\mu \nu}=\frac{\delta \tilde{\Gamma}^{1}}{\delta \tilde{a}_{\mu} \delta \tilde{a}_{\nu}}$ has to be transverse, while others not. One can now replace $A_{0}$ by a new transformation

$$
A=\left(\begin{array}{cc}
1 & 0 \\
i\left(k^{\mu}+T_{1_{21}}^{\mu}\right) & \left(1+T_{1_{22}}\right)
\end{array}\right)
$$

with $A^{-1}$ diagonalizing the 1-loop corrected bilinear form $T_{0}+T_{1}$

$$
T^{\prime}=A^{-1^{\dagger}}\left(T_{0}+T_{1}\right) A^{-1}=\left(\begin{array}{cc}
-k^{2} g^{\mu \nu}+T_{1_{11}}^{\mu \nu}+\Pi^{\prime \mu \nu} & 0 \\
0 & \left(1+T_{1_{22}}\right)^{-1}
\end{array}\right),
$$

where

$$
\Pi^{\prime \mu \nu}=k^{\mu} k^{\nu}-\frac{\left(k^{\mu}+T_{1_{21}}^{\mu}\right)\left(k^{\nu}+T_{1_{21}}^{\nu}\right)}{1+T_{1_{22}}} .
$$

We then conjecture that the formal leading order expansion of $\Pi^{\mu \nu}$ with respect to the coupling constant $e$ corresponds to the gauge fixing corrections to the 1-loop 1-PI photon two point function, i.e.

$$
\Pi_{\text {gf total }}^{\mu \nu}=\left.\Pi^{\prime \mu \nu}\left(k^{\mu} \rightarrow p^{\mu}\right)\right|_{e^{2}}=p^{\mu} p^{\nu} \cdot T_{1_{22}}-p^{\{\mu} T_{1_{21}}^{\nu\}} .
$$

And, as we shall see below, this relation/conjecture indeed holds.

\subsection{The action of the gauge and BRST-auxiliary fields and Feynman rules}

We define the noncommutative photon-auxiliary field action by using the first and the second order SW maps for the NC gauge field, $A_{\mu}^{(1)}$ and $A_{\mu}^{(2)}$, respectively:

$$
\begin{aligned}
S_{B-a_{\mu}}= & \int-\left(\partial^{\mu} B\right)\left(A_{\mu}^{(1)}+A_{\mu}^{(2)}\right) \\
= & \int-\left(\partial^{\mu} B\right) \frac{1}{2} \theta^{i j} a_{i} \star_{2}\left(\partial_{j} a_{\mu}+f_{j \mu}\right)+\left(\partial^{\mu} B\right) \frac{1}{8} \theta^{i j} \theta^{k l}\left(\left[a_{i} \partial_{j}\left(a_{k}\left(\partial_{l} a_{\mu}+f_{l \mu}\right)\right)\right]_{\star_{3^{\prime}}}\right. \\
& \left.-2\left[a_{i}\left(f_{j k} f_{\mu l}-a_{k} \partial_{l} f_{j \mu}\right)\right]_{\star_{3^{\prime}}}+\left[\left(\partial_{j} a_{\mu}+f_{j \mu}\right) a_{k}\left(\partial_{l} a_{i}+f_{l i}\right)\right]_{\star_{3^{\prime}}}\right)
\end{aligned}
$$

from where we obtain the corresponding photon-auxiliary field interaction vertices. Corresponding Feynman rules from the above action generate one-loop correction to the quadratic effective action and are given in appendix G. 


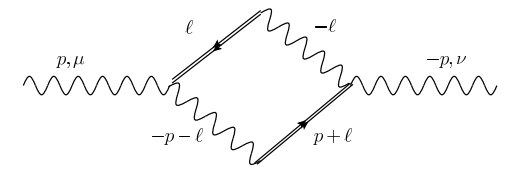

Figure 28. Bubble contribution to photon polarization: $B_{B-a_{\mu}}^{\mu \nu}(p)$, with $B-a_{\mu}$ propagators running in the loop in opposite directions.

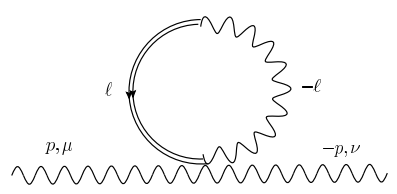

Figure 29. The 3gauge-1Bauxiliary fields tadpole contribution to photon polarization: $T_{B-a_{\mu}}^{\mu \nu}(p)$.

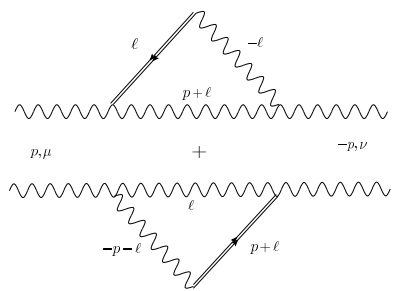

Figure 30. Two bubbles with mixing $a_{\mu}$ and $B-a_{\mu}$ propagators emerging from SW-interacting $\mathrm{U}(1)$.

\subsection{One-loop contributions to the photon effective action up to the quadratic order, from the BRST auxiliary field $B$}

Based on our vertex read-out convention, $\partial_{\mu}=i p_{\mu}$, we obtain the following correspondence rule between the matrix elements of one loop correction $T_{1}$ and the 1-PI loop diagrams

$$
\begin{aligned}
T_{1_{11}}^{\mu \nu} & =\Pi^{\mu \nu}+\Pi_{B-a_{\mu}}^{\mu \nu}, \\
\Pi_{B-a_{\mu}}^{\mu \nu} & =\text { figure } 25+\text { figure } 26+\text { figure } 28+\text { figure } 29+\text { figure } 30, \\
i T_{1_{21}}^{\mu} & =i \Pi^{\mu}+i \Pi_{\text {mix }}^{\mu}, \quad i \Pi^{\mu}=\text { figure } 31+\text { figure } 32, \\
T_{1_{22}} & =\text { figure } 33, \quad i \Pi_{\text {mix }}^{\mu}=\text { figure } 34 .
\end{aligned}
$$

Note that $\Pi^{\mu \nu}$ denotes all contributions from the classical action, which is the same as summing over all contributions to the photon two point function computed in sections $2-5$ and thus transverse. Explicit computation first revolves that

$$
\Pi_{B-a_{\mu}}^{\mu \nu}=0,
$$

thus the Slavnov-Taylor identity is actually trivially fulfilled.

The rest of the matrix elements listed in (7.19) are nonzero and boil down to the following expressions

$$
\Pi^{\mu}=\frac{e^{2}}{(4 \pi)^{2}}\left(p^{\mu} \Pi_{\mathrm{I}}+(\theta \theta p)^{\mu} \Pi_{\mathrm{II}}\right)
$$




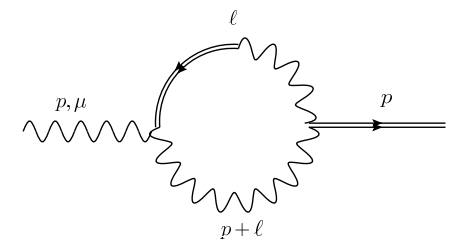

Figure 31. Bubble contribution to $i T_{1_{21}}$, with $B-a_{\mu}$-propagator in the loop.

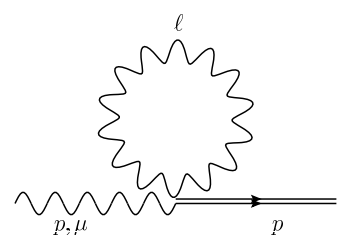

Figure 32. Tadpole contribution to $i T_{1_{21}}$, with photon-loop.

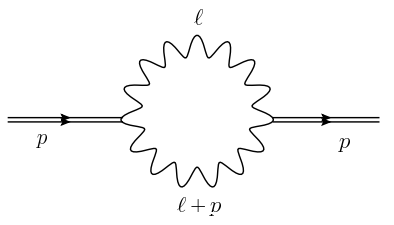

Figure 33. Bubble contribution to $T_{1_{22}}$, with photon-loop.

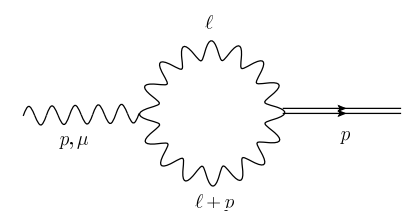

Figure 34. Bubble contribution $i \Pi_{\text {mix }}^{\mu}$ to $i T_{1_{21}}$, with photon-loop.

where

$$
\begin{aligned}
\Pi_{\mathrm{I}}= & \left.\left(4 \pi \mu^{2}\right)^{2-\frac{D}{2}}\left(p^{2}\right)^{\frac{D}{2}-2} \cdot(D-2) \cdot \Gamma\left(3-\frac{D}{2}\right) \mathrm{B}\left(\frac{D}{2}-1, \frac{D}{2}-1\right)\right|_{D \rightarrow 4-\epsilon}-\left(4 I_{K}^{0}+6 I_{H}\right), \\
\Pi_{\mathrm{II}}= & \frac{p^{2}}{(\theta p)^{2}}\left(\left.\left(4 \pi \mu^{2}\right)^{2-\frac{D}{2}}\left(p^{2}\right)^{\frac{D}{2}-2} \cdot(D-1) \cdot \Gamma\left(3-\frac{D}{2}\right) \mathrm{B}\left(\frac{D}{2}-1, \frac{D}{2}-1\right)\right|_{D \rightarrow 4-\epsilon}\right. \\
& \left.-\frac{1}{8}(\theta p)^{2} T_{-2}-\left(6 I_{K}^{0}+6 I_{H}\right)\right),
\end{aligned}
$$

and

$$
\Pi_{\text {mix }}^{\mu}=-\frac{e^{2}}{(4 \pi)^{2}}\left(p^{\mu}+(\theta \theta p)^{\mu} \frac{p^{2}}{(\theta p)^{2}}\right)\left(4+4 I_{H}\right) .
$$


Finally we have

$$
\begin{aligned}
T_{1_{22}=} & \frac{1}{2} \frac{e^{2}}{(4 \pi)^{2}}\left(\left.\left(4 \pi \mu^{2}\right)^{2-\frac{D}{2}}\left(p^{2}\right)^{\frac{D}{2}-2} \Gamma\left(2-\frac{D}{2}\right) \mathrm{B}\left(\frac{D}{2}-1, \frac{D}{2}-1\right)\right|_{D \rightarrow 4-\epsilon}\right. \\
& \cdot 4 \cdot\left((D-3)+\frac{p^{2}}{(\theta p)^{2}}\left(\operatorname{tr} \theta \theta+2(\theta \theta p)^{2}\right)\right)-\frac{1}{2} p^{2} \operatorname{tr} \theta \theta T_{-2} \\
& \left.-8 I_{K}^{0}-24 I_{H}-\frac{p^{2}}{(\theta p)^{2}} \cdot 2 \cdot\left(\operatorname{tr} \theta \theta(\theta p)^{2}\left(4 I_{K}^{0}+4 I_{H}\right)+(\theta \theta p)^{2}\left(8 I_{K}^{0}+4 I_{H}\right)\right)\right) .
\end{aligned}
$$

Next we start to verify our conjecture (7.17). First we derive the following relations from it,

$$
\Pi_{A}=(4 \pi)^{2} e^{-2} T_{1_{22}}-2 \Pi_{\mathrm{I}}, \quad \Pi_{B}=-\Pi_{\mathrm{II}}, \quad \Pi_{\mathrm{gf}}^{\mu \nu}=-p^{\{\mu} \Pi_{\mathrm{mix}}^{\nu\}} .
$$

One then immediately observes that the second and third relations do fulfill. As for the first one we can compute its right hand side

$$
\begin{aligned}
(4 \pi)^{2} & e^{-2} T_{1_{22}}-2 \Pi_{\mathrm{I}}=\frac{1}{2}\left(\left.\left(4 \pi \mu^{2}\right)^{2-\frac{D}{2}}\left(p^{2}\right)^{\frac{D}{2}-2} \Gamma\left(2-\frac{D}{2}\right) \mathrm{B}\left(\frac{D}{2}-1, \frac{D}{2}-1\right)\right|_{D \rightarrow 4-\epsilon}\right. \\
& \cdot 4 \cdot\left((D-3)+\frac{p^{2}}{(\theta p)^{2}}\left(\operatorname{tr} \theta \theta+2(\theta \theta p)^{2}\right)\right)-\frac{1}{2} p^{2} \operatorname{tr} \theta \theta T_{-2} \\
& \left.-8 I_{K}^{0}-24 I_{H}-\frac{p^{2}}{(\theta p)^{2}} \cdot 2 \cdot\left(\operatorname{tr} \theta \theta(\theta p)^{2}\left(4 I_{K}^{0}+4 I_{H}\right)+(\theta \theta p)^{2}\left(8 I_{K}^{0}+4 I_{H}\right)\right)\right) \\
& -2\left(\left.\left(4 \pi \mu^{2}\right)^{2-\frac{D}{2}}\left(p^{2}\right)^{\frac{D}{2}-2} \cdot(D-2) \cdot \Gamma\left(3-\frac{D}{2}\right) \mathrm{B}\left(\frac{D}{2}-1, \frac{D}{2}-1\right)\right|_{D \rightarrow 4-\epsilon}\right. \\
& \left.-\left(4 I_{K}^{0}+6 I_{H}\right)\right) \\
= & \frac{1}{2}\left(\left.\left(4 \pi \mu^{2}\right)^{2-\frac{D}{2}}\left(p^{2}\right)^{\frac{D}{2}-2} \Gamma\left(2-\frac{D}{2}\right) \mathrm{B}\left(\frac{D}{2}-1, \frac{D}{2}-1\right)\right|_{D \rightarrow 4-\epsilon}\right. \\
& \cdot 4 \cdot\left(-1+\frac{p^{2}}{(\theta p)^{2}}\left(\operatorname{tr} \theta \theta+2(\theta \theta p)^{2}\right)\right)-\frac{1}{2} p^{2} \operatorname{tr} \theta \theta T_{-2} \\
& \left.+8 I_{K}^{0}-\frac{p^{2}}{(\theta p)^{2}} \cdot 2 \cdot\left(\operatorname{tr} \theta \theta(\theta p)^{2}\left(4 I_{K}^{0}+4 I_{H}\right)+(\theta \theta p)^{2}\left(8 I_{K}^{0}+4 I_{H}\right)\right)\right)=\Pi_{A},
\end{aligned}
$$

which is in agreement with (6.7). Thus the conjectured relation (7.17) is proven.

Retrospectively, we see that the gauge fixing contribution to the 1-loop correction is a shift from the on-shell value of the gauge fixing functional. Therefore it does not need to be transverse. The fact that the pure gauge fixing and mixing contributions satisfies (7.17) independently is because the former can be considered as gauge fixing to a free U(1) gauge theory.

Finally let's briefly discuss the divergences in the gauge fixing configuration(s). Using the results from the appendix B we can see that $\Pi_{\mathrm{gf}_{\text {total }}}^{\mu \nu}$ contains no quadratic IR divergent 
term, therefore the quadratic IR divergence cancelation we found from the prior sections are also preserved under this gauge fixing choice. We can also extract the UV \& logarithmic divergence at the $D \rightarrow 4-\epsilon$ limit

$$
\begin{aligned}
& \left.\Pi_{A}\right|_{\mathrm{UV}}=-\left(2-\frac{p^{2}}{(\theta p)^{2}}\left(3 \operatorname{tr} \theta \theta(\theta p)^{2}+4(\theta \theta p)^{2}\right)\right)\left(\frac{2}{\epsilon}+\ln \left(\mu^{2}(\theta p)^{2}\right)\right), \\
& \left.\Pi_{B}\right|_{\mathrm{UV}}=-\frac{7}{2} \frac{p^{2}}{(\theta p)^{2}}\left(\frac{2}{\epsilon}+\ln \left(\mu^{2}(\theta p)^{2}\right)\right),
\end{aligned}
$$

while the $\Pi_{\text {gf mix }}^{\mu \nu}$ is finite at this limit. We conclude our analysis by listing of all UV plus logarithmic divergences in $\Pi_{\text {total }}^{\mu \nu}=\Pi^{\mu \nu}+\Pi_{\text {gftotal }}^{\mu \nu}$, which can be decomposed into seven symmetric tensor structures

$$
\begin{aligned}
\Pi_{\text {total }}^{\mu \nu} \mid \mathrm{UV}= & \frac{e^{2}}{(4 \pi)^{2}}\left(g^{\mu \nu} \cdot \Xi_{1}+p^{\mu} p^{\nu} \cdot \Xi_{2}+(\theta p)^{\mu}(\theta p)^{\nu} \cdot \Xi_{3}\right. \\
& +\left[g^{\mu \nu}(\theta p)^{2}-(\theta \theta)^{\mu \nu} p^{2}+p^{\{\mu}(\theta \theta p)^{\nu\}}\right] \Xi_{4} \\
& +\left[(\theta \theta)^{\mu \nu}(\theta p)^{2}+(\theta \theta p)^{\mu}(\theta \theta p)^{\nu}\right] \Xi_{5}+(\theta p)^{\{\mu}(\theta \theta \theta p)^{\nu\}} \Xi_{6} \\
& \left.+p^{\{\mu}(\theta \theta p)^{\nu\}} \cdot \Xi_{7}\right) .
\end{aligned}
$$

Explicit computation then yields

$$
\begin{aligned}
& \left.\Xi_{1}\right|_{\mathrm{UV}}=p^{2}\left(\frac{4}{3}-\frac{4}{3} n_{f}-\frac{1}{3} n_{s}+\frac{p^{2}}{(\theta p)^{4}}\left(3 \operatorname{tr} \theta \theta(\theta p)^{2}+4(\theta \theta p)^{2}\right)\right)\left(\frac{2}{\epsilon}+\ln \left(\mu^{2}(\theta p)^{2}\right)\right) \\
& \left.\Xi_{2}\right|_{\mathrm{UV}}=\left(-2-\frac{4}{3}+\frac{4}{3} n_{f}+\frac{1}{3} n_{s}\right)\left(\frac{2}{\epsilon}+\ln \left(\mu^{2}(\theta p)^{2}\right)\right) \\
& \left.\Xi_{3}\right|_{\mathrm{UV}}=\left.B_{2}\right|_{\mathrm{UV}}=2 \frac{p^{2}}{(\theta p)^{2}}\left(2-\frac{p^{2}(\operatorname{tr} \theta \theta)}{(\theta p)^{2}}\right)\left(\frac{2}{\epsilon}+\ln \left(\mu^{2}(\theta p)^{2}\right)\right) \\
& \left.\Xi_{4}\right|_{\mathrm{UV}}=\left.B_{3}\right|_{\mathrm{UV}}=2 \frac{p^{2}}{(\theta p)^{2}}\left(\frac{2}{\epsilon}+\ln \left(\mu^{2}(\theta p)^{2}\right)\right) \\
& \left.\Xi_{5}\right|_{\mathrm{UV}}=\left.B_{4}\right|_{\mathrm{UV}}=-4 \frac{p^{4}}{(\theta p)^{4}}\left(\frac{2}{\epsilon}+\ln \left(\mu^{2}(\theta p)^{2}\right)\right) \\
& \left.\Xi_{6}\right|_{\mathrm{UV}}=\left.B_{5}\right|_{\mathrm{UV}}=4 \frac{p^{4}}{(\theta p)^{4}}\left(\frac{2}{\epsilon}+\ln \left(\mu^{2}(\theta p)^{2}\right)\right) \\
& \left.\Xi_{7}\right|_{\mathrm{UV}}=\left.\Pi_{B}\right|_{\mathrm{UV}}=-\frac{7}{2} \frac{p^{2}}{(\theta p)^{2}}\left(\frac{2}{\epsilon}+\ln \left(\mu^{2}(\theta p)^{2}\right)\right) .
\end{aligned}
$$

\section{Summary and discussion}

In this paper we have computed the one-loop contributions to all the propagators of the noncommutative super Yang-Mills $\mathrm{U}(1)$ theory with $\mathcal{N}=1,2$ and 4 supersymmetry and defined by the means of the $\theta$-exact Seiberg-Witten map. We have shown that for $\mathcal{N}=1,2$ and 4 the quadratic noncommutative IR divergence,

$$
\frac{(\theta p)^{\mu}(\theta p)^{\nu}}{(\theta p)^{4}}
$$


a trade-mark of noncommutative gauge theories - which occur in the bosonic, fermionic and scalar contributions to photon propagator cancel each other, rendering a photon propagator free of them as befits Supersymmetry. Indeed, from (4.3) for $\mathcal{N}=1,2$, 4 , one gets:

$$
\begin{aligned}
\mathcal{N} & =1: n_{f}=1, n_{s}=0, \\
\mathcal{N} & =2: n_{f}=2, n_{s}=2, \\
\mathcal{N} & =4: n_{f}=4, n_{s}=6, \\
\left.\Pi_{\text {total }}^{\mu \nu}(p)\right|_{\text {IR }} & =\frac{e^{2}}{(4 \pi)^{2}}\left[\left(\frac{32}{3}+\frac{64}{3}\right)-32 \cdot n_{f}+16 \cdot n_{s}\right] \frac{(\theta p)^{\mu}(\theta p)^{\nu}}{(\theta p)^{4}}=0 .
\end{aligned}
$$

This cancellation, occuring in the case at hand, is nontrivial since Supersymmetry acts nonlinearly - see (2.16) — on the ordinary fields. Let us recall that the cancellation of quadratic noncommutative IR divergences is also a feature of noncommutative super YangMills theories when formulated in terms of noncommutative fields [1-5]. Hence, our result concerning the cancellation of the quadratic noncommutative IR divergences really points into the direction that the $\theta$-exact Seiberg-Witten map really provides quantum duals of the same underlying theory.

We have shown — see (4.11) and (5.6) - that the characteristic quadratic noncommutative IR divergences,

$$
\frac{1}{(\theta p)^{2}}
$$

which arise in the individual contributions to the one-loop propagators of the scalar fields in the $\mathcal{N}=2$ and 4 Supersymmetry, also cancel each other at the end of the day. The same holds for the photino field as well.

Since the previous cancellations occur both in the ordinary Feynman gauge and in the noncommutative Feynman gauge - see section 7, our computations further indicate that the cancellation is robust against changing the gauge fixing and may have real physical, and therefore gauge invariant, content. Let us recall that independence of gauge-fixing parameter of the cancellation of noncommutative IR divergences in the dual theory, i.e., in $\mathcal{N}=1 \mathrm{U}(1)$ super Yang-Mills theory formulated in terms of the noncommutative fields, has been shown to hold - see ref. [2].

In this paper we have also worked out explicitly the one-loop UV divergent contributions - which show as poles at $D=4$ - to all propagator of the theory: see (2.49), (2.33)-(2.34), (4.13), (4.16), (5.7), (5.12) and (7.30)-(7.37). It is noticeable that the pole parts displayed in the equations we have just quoted contain non-polynomial, i.e., non-local, terms whose denominator isa power of $\theta p$. With respect to this we would like to point out that, in keeping with Weinberg's power counting theorem [24], Feynman integrals whose degree of UV divergence are not the same along all directions are liable to give rise to the pole contributions which are non-polynomial. This is exactly our situation since our integrands contain factors of the type

$$
\frac{1}{\left(q^{2}\right)^{n}\left((q+p)^{2}\right)^{m}(q \theta p)^{s}}, \quad s=1,2,
$$


and these factors approach zero as $\Lambda^{(-2 n-2 m-s)}$ along the direction parallel to $\theta p$, and as $\Lambda^{(-2 n-2 m)}$ along any direction orthogonal to $\theta p$. Hence UV divergences with a nonpolynomial dependence on the momenta may occur and our computations show that indeed they do occur. We would like to recall that a similar situation, - i.e. the non-polynomial UV divergences - happens in ordinary Yang-Mills theories in the light-cone gauge [25, 26].

Now, the UV divergences of two-point functions are in general gauge dependent quantities. We have verified that this is so in our case by computing the one-loop propagator of the gauge field both in the ordinary Feynman gauge and in the noncommutative Feynman gauge - see section 6. The result for the first type of gauge is in (2.33)-(2.34) and in (7.30)-(7.37) for the second type of gauge fixing term: their differences stand out. Hence, extracting gauge invariant information from the UV divergences is our next challenge along this line of research and it will require the computation of three and higher point functions.

Let us finally remark that UV/IR mixing effects also work for the nonpolynomial UV divergent contributions we have obtained. Indeed, as seen in (2.49), (2.33)-(2.34), (4.13), (4.16), (5.7), (5.12) and (7.30)-(7.37) every pole in $2 / \epsilon$ comes hand in hand with the logarithmic noncommutative IR divergence $\ln \left(\mu^{2}(\theta p)^{2}\right)$. The reader is referred to the final part of appendix $\mathrm{B}$ for further information regarding this issue.

\section{Acknowledgments}

The work by C.P. Martin has been financially supported in part by the Spanish MINECO through grant FPA2014-54154-P. J.Y. has been fully supported by Croatian Science Foundation under Project No. IP-2014-09-9582. The work J.T. is conducted under the European Commission and the Croatian Ministry of Science, Education and Sports Co-Financing Agreement No. 291823. In particular, J.T. acknowledges project financing by the Marie Curie FP7-PEOPLE-2011-COFUND program NEWFELPRO: Grant Agreement No. 69, and Max-Planck-Institute for Physics, and W. Hollik for hospitality. J.T. would also like to acknowledge L. Alvarez-Gaume for fruitful discussions and CERN Theory Division, where part of this work was conducted, for hospitality. We would like to acknowledge the COST Action MP1405 (QSPACE). We would also like to thank J. Erdmenger, W. Hollik and A.Ilakovac, for fruitful discussions. A great deal of computation was done by using MATHEMATICA 8.0Mathematica [28] plus the tensor algebra package xACT [29]. Special thanks to A. Ilakovac and D. Kekez for the computer software and hardware support.

\section{A Seiberg-Witten differential equations for the SYM U(1)}

Let $\Phi$ be a noncommutative field, either boson or fermion, in $d$ dimensions, which gauge transforms under the adjoint of $\mathrm{U}(\mathrm{N})$. Then its NC BRST transformation reads

$$
s_{\mathrm{NC}} \Phi=-i[\Phi, \Omega]
$$

where $\Omega$ is the noncommutative $\mathrm{U}(\mathrm{N})$ ghost field in $d$ dimensions that parametrizes the noncommutative BRST transformations of the $\mathrm{U}(\mathrm{N})$ gauge field $A_{\mu}$ in $d$ dimensions:

$$
s_{\mathrm{NC}} A_{\mu}=\partial_{\mu} \Omega-i\left[A_{\mu}, \Omega\right], \quad s_{\mathrm{NC}} \Omega=i \Omega \star \Omega .
$$


Let $\phi$ and $a_{\mu}$ be an ordinary matter and gauge fields in $d$ dimensions which take values in the Lie algebra of $\mathrm{U}(\mathrm{N})$ in the fundamental representation. Let the BRST transformations of $\phi$ and $a_{\mu}$ be

$$
s \phi=-i[\phi, \omega], \quad s a_{\mu}=\partial_{\mu} \omega-i\left[a_{\mu}, \omega\right], \quad s \omega=i \omega \cdot \omega,
$$

where $\omega$ is the ordinary ghost field in $d$ dimensions which also takes values in Lie algebra of $\mathrm{U}(\mathrm{N})$ in the fundamental representation. Then, the SW map $\left\{\Omega\left[e \cdot a, \omega ; \theta^{\mu \nu}\right], A_{\mu}[e\right.$. $\left.\left.a ; \theta^{\mu \nu}\right], \Phi\left[e \cdot a, \phi ; \theta^{\mu \nu}\right]\right\}$ is a solution to the problem

$$
\begin{aligned}
s \Omega & =i \Omega \star \Omega, & \Omega\left[e \cdot a, \omega ; \theta^{i j}\right. & =0]=\omega, \\
s A_{\mu} & =\partial_{\mu} \Omega-i\left[A_{\mu} \star \star \Omega\right], & A_{\mu}\left[e \cdot a ; \theta^{i j}\right. & =0]=e \cdot a_{\mu}, \\
s \Phi & =-i[\Phi *, \Omega], & \Phi\left[e \cdot a, \phi ; \theta^{i j}\right. & =0]=\phi .
\end{aligned}
$$

It is known that the following set of differential equations - called the Seiberg-Witten differential equations $[11,27]$ - furnish a solution to the problem in the system of equations (A.4):

$$
\begin{aligned}
\frac{d}{d t} \Omega & =-\frac{1}{4} \theta^{i j}\left\{A_{i} \stackrel{\star,}{,} \partial_{j} \Omega\right\}, & \Omega[t=0] & =\omega, \\
\frac{d}{d t} A_{\mu} & =-\frac{1}{4} \theta^{i j}\left\{A_{i} \stackrel{\star \star,}{,} \partial_{j} A_{\mu}+F_{j \mu}\right\}, & A_{\mu}[t=0] & =e \cdot a_{\mu}, \\
\frac{d}{d t} \Phi & =-\frac{1}{4} \theta^{i j}\left\{A_{i} \stackrel{\star, t}{,} \partial_{j} \Phi+\mathcal{D}_{j} \Phi\right\}, & \Phi[t=0] & =\phi .
\end{aligned}
$$

Note that $\mu$ runs from 0 to $d-1$, while $i$ runs from 1 to $d-1$, respectively.

Now we show how a solution to the previous problem can be obtained by solving Seiberg-Witten differential equations for a $\mathrm{U}(\mathrm{N})$ gauge field in $d+1$ dimensions. Let $A_{M}=\left(A_{\mu}, A_{d}\right)$ be a noncommutative gauge field in $d+1$ dimensions and in the fundamental representation of $\mathrm{U}(\mathrm{N})$ and let $\hat{\Omega}$ denote the corresponding noncommutative ghost field. Then the Seiberg-Witten differntial equations for $A_{M}$ and $\hat{\Omega}$ read

$$
\begin{aligned}
\frac{d}{d t} \hat{\Omega} & =-\frac{1}{4} \theta^{I J}\left\{A_{I} \stackrel{{ }^{\star}}{,} \partial_{J} \hat{\Omega}\right\}, & \hat{\Omega}[t=0] & =\hat{\omega}, \\
\frac{d}{d t} A_{M} & =-\frac{1}{4} \theta^{I J}\left\{A_{I} \stackrel{\star_{t}}{,} \partial_{J} A_{\mu}+F_{J M}\right\}, & & A_{M}[t=0]=e \cdot a_{M},
\end{aligned}
$$

where $I$ and $J$ run from 1 to $d$, and $a_{M}=\left(a_{\mu}, a_{d+1}\right)$ and $\hat{\omega}$ are the corresponding ordinary fields in $d+1$ dimensions.

Let us assume that the coordinate $X^{d}$ commutes with all the others, i.e., $\theta^{I J}$ is such that $\theta^{I d}=0$. Now, let $A_{M}\left[e \cdot a_{M}^{\prime}, ; \theta^{I J}\right]$ and $\hat{\Omega}\left[e \cdot a_{M}^{\prime}, \hat{\omega} ; \theta^{I J}\right]$ be the solution to (A.6) and let us take now $a_{M}$ and $\hat{\omega}$ to be independent of $x^{d}$, so that $A_{M}\left[e \cdot a_{M}^{\prime}, ; \theta^{I J}\right]$ and $\hat{\Omega}\left[e \cdot a_{M}^{\prime}, \hat{\omega} ; \theta^{I J}\right]$ become independent of $x^{d}$. Now, for these $A_{M}\left[e \cdot a_{M}^{\prime}, ; \theta^{i j}\right]$ and $\hat{\Omega}\left[e \cdot a_{M}^{\prime}, \hat{\omega} ; \theta^{i j}\right]$ the SW differential equations in (A.6) boil down to

$$
\begin{aligned}
\frac{d}{d t} \hat{\Omega} & =-\frac{1}{4} \theta^{i j}\left\{A_{i} \stackrel{\star t}{,} \partial_{j} \hat{\Omega}\right\}, & \hat{\Omega}[t=0] & =\hat{\omega}, \\
\frac{d}{d t} A_{\mu} & =-\frac{1}{4} \theta^{i j}\left\{A_{i}{ }^{\star t}, \partial_{j} A_{\mu}+F_{j \mu}\right\}, & A_{\mu}[t=0] & =e \cdot a_{\mu}, \\
\frac{d}{d t} A_{d} & =-\frac{1}{4} \theta^{i j}\left\{A_{i}{ }^{\star t}, \partial_{j} A_{d}+\mathcal{D}_{j} A_{d}\right\}, & A_{d}[t=0] & =e \cdot a_{d},
\end{aligned}
$$


where we have taken into account that $F_{\mu d}=\partial_{\mu} A_{d}-i\left[A_{\mu}, A_{d}\right]=\mathcal{D}_{\mu} A_{d}$, for $A_{d}$ does not depend on $x^{d}$. It is plain that if we replace $A_{d}$ with $\Phi$ and $e \cdot a_{d}$ with $\phi$ in (A.7), one obtains (A.5). We thus conclude that the SW map for $\Phi$ can be obtained from the SW map $A_{d}\left[e \cdot a_{\mu}, e \cdot a_{d} ; \theta^{i j}\right]$ that solves (A.6) by replacing $e \cdot a_{d}$ with $\phi$. We also deduce the following relation between the gauge field strength $F_{\mu d}$ and the covariant derivative $\mathcal{D}_{\mu}$

$$
\mathcal{D}_{\mu}\left[A_{\nu}\right] \Phi=\left.F_{\mu d}\left[e \cdot a_{\mu}, e \cdot a_{d} ; \theta^{i j}\right]\right|_{e \cdot a_{d} \rightarrow \phi}
$$

Next, let $A_{\mu}\left[a_{\nu} ; \theta^{i j}\right], \Lambda_{\alpha}\left[a_{\mu}, \lambda_{\alpha} ; \theta^{i j}\right]$ and $D^{(n c)}\left[a_{\mu}, D ; \theta^{i j}\right]$ be $\theta$-exact Seiberg-Witten maps given by the following expansions in terms of the coupling constant $e$ :

$$
\begin{aligned}
A_{\mu}\left[a_{\nu} ; \theta^{i j}\right] & =e\left(a_{\mu}+e A_{\mu}^{(1)}\left[a_{\nu} ; \theta^{i j}\right]+e^{2} A_{\mu}^{(2)}\left[a_{\nu} ; \theta^{i j}\right]\right)+\mathcal{O}\left(e^{4}\right), \\
\Lambda_{\alpha}\left[a_{\nu}, \lambda_{\alpha} ; \theta^{i j}\right] & =\lambda_{\alpha}+e \Lambda_{\alpha}^{(1)}\left[a_{\nu}, \lambda_{\alpha} ; \theta^{i j}\right]+e^{2} \Lambda_{\alpha}^{(2)}\left[a_{\nu}, \lambda_{\alpha} ; \theta^{i j}\right]+\mathcal{O}\left(e^{3}\right), \\
D^{(n c)}\left[a_{\nu}, D ; \theta^{i j}\right] & =D+e D^{(1)}\left[a_{\nu}, D ; \theta^{i j}\right]+e^{2} D^{(2)}\left[a_{\nu}, D ; \theta^{i j}\right]+\mathcal{O}\left(e^{3}\right) .
\end{aligned}
$$

Taking the $n$-th variations, of the $m$-th order of the NC gauge field $A_{\mu}$, its supersymmetric fermion partner $\Lambda_{\alpha}$ and of the NC auxiliary field $D^{(n c)}$, we obtain the following expressions

$$
\begin{aligned}
\delta^{n} A_{\mu}^{(m)} & =A_{\mu}^{(m)}\left[a_{\nu}+\delta^{n} a^{\nu} ; \theta^{i j}\right]-A_{\mu}^{(m)}\left[a_{\nu} ; \theta^{i j}\right]+\mathcal{O}\left(\xi^{2}\right), \\
\delta^{n} \Lambda_{\alpha}^{(m)}\left[a_{\nu}, \lambda_{\alpha} ; \theta^{i j}\right] & =\Lambda_{\alpha}^{(m)}\left[a_{\nu}+\delta^{n} a^{\nu}, \lambda_{\alpha}+\delta^{n} \lambda_{\alpha} ; \theta^{i j}\right]-\Lambda_{\alpha}^{(m)}\left[a_{\nu}, \lambda_{\alpha} ; \theta^{i j}\right]+\mathcal{O}\left(\xi^{2}\right), \\
\delta^{n} D^{(m)}\left[a_{\nu}, D ; \theta^{i j}\right] & =D^{(m)}\left[a_{\nu}+\delta^{n} a^{\nu}, D+\delta^{n} D ; \theta^{i j}\right]-D^{(m)}\left[a_{\nu}, D ; \theta^{i j}\right]+\mathcal{O}\left(\xi^{2} x\right) .
\end{aligned}
$$

Here $m=1,2$, while $\delta^{n} a^{\nu}, \delta^{n} \lambda_{\alpha}$ and $\delta^{n} D$ for $n=0,1$, have been given in (2.20).

\section{B Integrals}

A fairly large number of special function integrals occur in studying NCQFT, with or without SW map. Some description of the integrals relevant to this work was given in [16], where we used a set of seven special function integrals to present the nonplanar part of the bubble integrals at $D=4$. During this work and our prior study on NC tadpole integrals [18] we studied additional new integrals and found some new relations among all of them. Here we present a new list of five integrals which are used to present all loop integral results in the main text.

The original set of seven integrals include four Bessel K-function integrals and three integrals over a function $H[z]$ which can be expressed in terms of hypergeometric functions

$$
\begin{aligned}
H[z]= & \lim _{D \rightarrow 4}\left[\left(\frac{z}{2}\right)^{D-2} \Gamma\left(1-\frac{D}{2}\right){ }_{1} F_{2}\left(\frac{1}{2} ; \frac{3}{2}, \frac{D}{2} ;\left(\frac{z}{2}\right)^{2}\right)\right. \\
& \left.+\frac{1}{3-D} \cdot \Gamma\left(\frac{D}{2}-1\right){ }_{1} F_{2}\left(\frac{3-D}{2} ; \frac{4-D}{2}, \frac{5-D}{2} ;\left(\frac{z}{2}\right)^{2}\right)\right] .
\end{aligned}
$$


Below is a list of these integrals

$$
\begin{aligned}
I_{1} & =\int_{0}^{1} d x\left(x(1-x) p^{2}\right)^{\frac{1}{2}}\left((\theta p)^{2}\right)^{-\frac{1}{2}} K_{1}\left[\left(x(1-x) p^{2}(\theta p)^{2}\right)^{\frac{1}{2}}\right] \\
I_{2} & =\int_{0}^{1} d x K_{0}\left[\left(x(1-x) p^{2}(\theta p)^{2}\right)^{\frac{1}{2}}\right] \\
I_{3} & =\int_{0}^{1} d x x K_{0}\left[\left(x(1-x) p^{2}(\theta p)^{2}\right)^{\frac{1}{2}}\right] \\
I_{4} & =\int_{0}^{1} d x x^{2} K_{0}\left[\left(x(1-x) p^{2}(\theta p)^{2}\right)^{\frac{1}{2}}\right] \\
I_{5} & =\int_{0}^{1} d x H\left[\left(x(1-x) p^{2}(\theta p)^{2}\right)^{\frac{1}{2}}\right] \\
I_{6} & =\int_{0}^{1} d x x H\left[\left(x(1-x) p^{2}(\theta p)^{2}\right)^{\frac{1}{2}}\right] \\
I_{7} & =\int_{0}^{1} d x x^{2} H\left[\left(x(1-x) p^{2}(\theta p)^{2}\right)^{\frac{1}{2}}\right] .
\end{aligned}
$$

Later it is revolved that $I_{1}$ is directly related to the tadpole integrals

$$
\begin{aligned}
A_{1} & =\int \frac{d^{D} k}{(2 \pi)^{D}} \frac{(k \theta p)^{2}}{k^{2}} f_{\star 2}(k, p)^{2}=-8 \frac{1}{(4 \pi)^{2}} \frac{1}{(\theta p)^{2}} \\
& =-\frac{1}{(4 \pi)^{2}}\left(8 I_{1}+p^{2}\left(4 I_{2}-12 I_{3}+8 I_{4}\right)\right),
\end{aligned}
$$

and

$$
\begin{aligned}
A_{2} & =\int \frac{d^{D} k}{(2 \pi)^{D}} \frac{(k \cdot p)^{2}}{k^{2}} f_{\star_{2}}(k, p)^{2}=\frac{8}{3} \frac{1}{(4 \pi)^{2}} \frac{p^{2}}{(\theta p)^{4}} \\
& =\frac{1}{3} \frac{1}{(4 \pi)^{2}} \frac{p^{2}}{(\theta p)^{4}}\left(8 I_{1}+p^{2}\left(12 I_{2}-92 I_{3}+104 I_{4}\right)+4 p^{2}\left(3 I_{5}-26 I_{6}+32 I_{7}\right)\right) .
\end{aligned}
$$

It is convenient to use the tadpole integral in lieu of the integral $I_{1}$ since the tadpole integral is quadratic IR divergent only. We select

$$
\begin{aligned}
T_{0} & =\frac{1}{4}(4 \pi)^{2} A_{1}=(4 \pi)^{2} \int \frac{d^{D} k}{(2 \pi)^{D}} k^{-2} \sin ^{2} \frac{k \theta p}{2}=-2 \frac{1}{(\theta p)^{2}} \\
& =-2 I_{1}-p^{2}\left(I_{2}-3 I_{3}+2 I_{4}\right),
\end{aligned}
$$

to fulfill this task. We can also extract an identity

$$
\left(4 I_{2}-12 I_{4}\right)+\left(5 I_{5}-16 I_{7}\right)=0
$$


from the relation $A_{1}=-3 A_{2} p^{-2}(\theta p)^{2}$. The other two useful relations are:

$$
I_{2}-2 I_{3}=I_{5}-2 I_{6}=0 .
$$

Using these three relations we can reduce the rest six integrals $I_{2-7}$ to three, which we choose to be

$$
\begin{aligned}
& I_{K}^{0}=I_{2}=\int_{0}^{1} d x K_{0}\left[\left(x(1-x) p^{2}(\theta p)^{2}\right)^{\frac{1}{2}}\right], \\
& I_{K}^{1}=I_{3}-I_{4}=\int_{0}^{1} d x x(1-x) K_{0}\left[\left(x(1-x) p^{2}(\theta p)^{2}\right)^{\frac{1}{2}}\right], \\
& I_{H}=I_{5}=\int_{0}^{1} d x H\left[\left(x(1-x) p^{2}(\theta p)^{2}\right)^{\frac{1}{2}}\right] .
\end{aligned}
$$

Using the generalized power series expansions in the vicinity of $z=0,{ }^{3}$

$$
K_{0}[z]=-\sum_{k=0}^{\infty} \frac{1}{\Gamma[k+1]^{2}}\left(\frac{z}{2}\right)^{2 k}\left(\ln \frac{z}{2}-\psi(k+1)\right),
$$

and

$$
\begin{aligned}
H[z]= & -1+\sum_{k=0}^{\infty} \frac{\Gamma\left(k+\frac{3}{2}\right)}{\Gamma\left(k+\frac{5}{2}\right) \Gamma(k+1) \Gamma(k+2)}\left(\frac{z}{2}\right)^{2 k+2} \\
& \cdot\left(\ln \frac{z}{2}+\frac{1}{2} \psi\left(k+\frac{1}{2}\right)-\frac{1}{2} \psi(k+1)-\frac{1}{2} \psi\left(k+\frac{3}{2}\right)-\frac{1}{2} \psi(k+2)\right),
\end{aligned}
$$

it is not difficult to see that the integrals $T_{0}, I_{K}^{0}, I_{K}^{1}$ and $I_{H}$ bear distinctive asymptotic behavior in the IR regime. The $T_{0}$ is quadratically IR divergent by definition, while $I_{K}^{0}$ and $I_{K}^{1}$ carry the dual logarithmic noncommutative IR divergence (logarithmic UV/IR mixing) $\ln \left(p^{2}(\theta p)^{2}\right)$, with coefficients $-1 / 2$ and $-1 / 12$, respectively. The last integral $I_{H}$ is finite at the IR limit.

A new type of tadpole integral, which is UV divergent at the $D \rightarrow 4-\epsilon$ limit occurs repeatedly in the NC Feynman gauge computation part of this work. Here we provide an account of its evaluation. This new tadpole, denoted as $T_{-2}$, bears a very simple form

$$
T_{-2}=(4 \pi)^{2} \mu^{4-D} \int \frac{d^{D} k}{(2 \pi)^{D}} \frac{1}{k^{2}} f_{\star_{2}}(k, p)^{2} .
$$

On the other hand, it turns out that $T_{-2}$ is not that simple to evaluate. Two usual regularization methods used before, turning tadpole to bubble or using the $n$-nested zero regulator, do not function here. The first one produces divergent special function integrals while the second contains unfavorable powers of the regulator. The parametrization discussed in the

\footnotetext{
${ }^{3} \psi(z)=\frac{d}{d z} \ln \Gamma(z)$ denotes the zeroth order polygamma function.
} 
first section of this note offers us an alternative way to handle this problem. Using that parametrization we can express $T_{-2}$ as

$$
\begin{aligned}
T_{-2} & =(4 \pi)^{2} \mu^{4-D} \int \frac{d^{D-1} \ell}{(2 \pi)^{D-1}} \int_{-\infty}^{+\infty} \frac{d x}{2 \pi} \frac{1}{\ell^{2}+x^{2}} \frac{4 \sin ^{2} \frac{|\theta p|}{2} x}{x^{2}(\theta p)^{2}} \\
& =(4 \pi)^{2} \mu^{4-D} \int \frac{d^{D-1} \ell}{(2 \pi)^{D-1}} \frac{1}{(\theta p)^{2}}\left(-\frac{1}{|\ell|^{3}}+\frac{2|\theta p|}{|\ell|^{2}}+\frac{e^{-|\ell||\theta p|}}{|\ell|^{3}}\right) .
\end{aligned}
$$

Unlike $A_{2}$, here we can only neglect the second term in the last parenthesis because the first and last exceed the minimal value of the loop momenta power $m=-2$ in the dimensional regularization prescription. Then one can introduce one more integrand $y$ to make the first and last terms into one

$$
\begin{aligned}
T_{-2} & =(4 \pi)^{2} \mu^{4-D} \int \frac{d^{D-1} \ell}{(2 \pi)^{D-1}} \frac{1}{(\theta p)^{2}}\left(-\frac{1}{|\ell|^{3}}+\frac{e^{-|\ell||\theta p|}}{|\ell|^{3}}\right) \\
& =-(4 \pi)^{2} \mu^{4-D} \int_{0}^{1} d y \int \frac{d^{D-1} \ell}{(2 \pi)^{D-1}} \frac{1}{|\theta p|} \frac{e^{-y|\ell||\theta p|}}{|\ell|^{2}} \\
& =-(4 \pi)^{\frac{5-D}{2}} \mu^{4-D} \frac{2}{\Gamma\left(\frac{D-1}{2}\right)} \int_{0}^{1} d y \int_{0}^{\infty} d l|\theta p|^{-1} l^{D-4} e^{-l y|\theta p|} \\
& =-(4 \pi)^{\frac{5-D}{2}} \mu^{4-D} \frac{2}{\Gamma\left(\frac{D-1}{2}\right)} \int_{0}^{1} d y|\theta p|^{2-D} y^{3-D} \Gamma(D-3) \\
& =-(4 \pi)^{\frac{5-D}{2}} \mu^{4-D} \frac{2}{\Gamma\left(\frac{D-1}{2}\right)}|\theta p|^{2-D} \frac{\Gamma(D-3)}{4-D} \\
& =\left(4 \pi \mu^{2}\right)^{\frac{4-D}{2}}\left(\frac{(\theta p)^{2}}{4}\right)^{1-\frac{D}{2}} \frac{\Gamma\left(\frac{D}{2}-2\right)}{D-3} .
\end{aligned}
$$

Finally, a familiar pattern emerges once we compute the $D \rightarrow 4$ limit

$$
T_{-2}=-\frac{4}{(\theta p)^{2}}\left(\frac{2}{4-D}+\ln \left(\mu^{2}(\theta p)^{2}\right)+\ln \pi+\gamma_{E}+2\right)+\mathcal{O}(4-D)
$$

Here we see the logarithmic UV/IR mixing taking place via a single integral.

In the end all loop integrals are expressed via usual planar integrals plus nonplanar integrals $T_{-2}, T_{0}, I_{K}^{0}, I_{K}^{1}$ and $I_{H}$. 
Figure 35. $\mathcal{N}=1$ photino propagator, eq. (B.2).

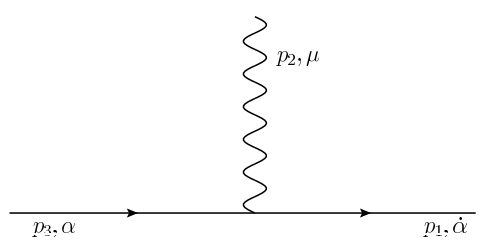

Figure 36. $\mathcal{N}=1$ photino-photon vertex: $V_{\rho}^{e^{1 \mu}}\left(p_{1}, p_{2}\right) ; p_{2}+p_{3}-p_{1}=0$

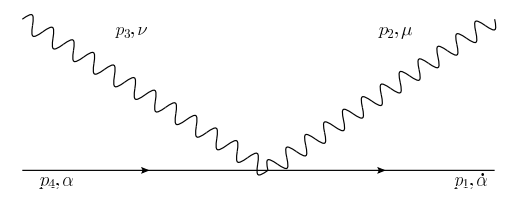

Figure 37. $\mathcal{N}=1$ photino-2photons vertex: $V_{\rho}^{e^{2 \mu \nu}}\left(p_{2}, p_{3}, p_{4}\right) ; p_{2}+p_{3}+p_{4}-p_{1}=0$.

\section{Photino-photon Feynman rules}

The $\mathcal{N}=1$ photino action $S^{\text {photino }},(2.19)$, in the momentum space reads

$$
\begin{aligned}
S^{\text {photino }}= & \int \frac{d^{4} p}{(2 \pi)^{4}} \bar{\lambda}_{\dot{\alpha}}(p) p_{\mu} \bar{\sigma}^{\mu \dot{\alpha} \alpha} \lambda_{\alpha}(p) \\
& +\int \prod_{i=1}^{4} \frac{d^{4} p_{i}}{(2 \pi)^{4}}(2 \pi)^{4} \delta\left(p_{1}-p_{2}-p_{3}\right) \bar{\lambda}_{\dot{\alpha}}\left(p_{1}\right) a_{\mu}\left(p_{2}\right) \lambda_{\alpha}\left(p_{3}\right) \bar{\sigma}^{\rho \dot{\alpha} \alpha} V_{\rho}^{e^{1 \mu}}\left[p_{1},-p_{2},-p_{3}\right] \\
& +\int \prod_{i=1}^{4} \frac{1}{2} \frac{d^{4} p_{i}}{(2 \pi)^{4}}(2 \pi)^{4} \delta\left(p_{1}-\sum_{j=2}^{4} p_{j}\right) \\
& \left.\cdot \bar{\lambda}_{\dot{\alpha}}\left(p_{1}\right) a_{\mu}\left(p_{2}\right) a_{\nu}\left(p_{3}\right) \lambda_{\alpha}\left(p_{4}\right) \bar{\sigma}^{\rho \dot{\alpha} \alpha} V_{\rho}^{e^{2 \mu \nu}}\left[p_{1},-p_{2},-p_{3},-p_{4}\right]+\mathcal{O}\left(e^{3}\right), \quad \text { (C. } 1\right)
\end{aligned}
$$

where all three terms above are represented by figures 35, 36, and 37. For photino propagator in particular, see [20]:

$$
\left\langle 0\left|T \lambda_{\alpha}(x) \bar{\lambda}_{\dot{\beta}}(y)\right| 0\right\rangle=\int \frac{d^{4} p}{(2 \pi)^{4}} \frac{i p_{\mu} \sigma_{\alpha \dot{\beta}}^{\mu}}{p^{2}+i \epsilon} e^{-i p(x-y)} .
$$

From the second line in (C.1) and figure 36 the photino-photon vertex reads as follows:

$$
\bar{\sigma}^{\rho \dot{\alpha} \alpha} V_{\rho}^{e^{1 \mu}}\left(p_{1}, p_{2}\right)=f_{\star_{2}}\left(p_{1}, p_{2}\right)\left(\bar{\sigma}^{\rho \dot{\alpha} \alpha} p_{2_{\rho}}\left(\theta p_{1}\right)^{\mu}-\bar{\sigma}^{\mu \dot{\alpha} \alpha}\left(p_{2} \theta p_{1}\right)-\left(\theta p_{2}\right)^{\mu} \bar{\sigma}^{\rho \dot{\alpha} \alpha} p_{1_{\rho}}\right),
$$

where $\left(p_{2}, \mu\right)$ is the photon incoming (momenta, index) and the fermion momentum $p_{1}$ flows through the vertex, as it should. 
From the third line in (C.1) and figure 37 the photino-2photons vertex reads as follows:

$$
\begin{aligned}
& \bar{\sigma}^{\rho \dot{\alpha} \alpha} V_{\rho}^{e^{2 \mu \nu}}\left(p_{2}, p_{3}, p_{4}\right)= \\
& -i\left(\left(\theta p_{2}\right)^{\mu}\left(\bar{\sigma}^{\rho \dot{\alpha} \alpha} p_{3_{\rho}}\left(\theta p_{4}\right)^{\nu}-\bar{\sigma}^{\nu \dot{\alpha} \alpha}\left(p_{3} \theta p_{4}\right)\right) f_{\star_{2}}\left(p_{1}, p_{2}\right) f_{\star_{2}}\left(p_{3}, p_{4}\right)\right. \\
& +\left(\theta p_{3}\right)^{\nu}\left(\bar{\sigma}^{\rho \dot{\alpha} \alpha} p_{2_{\rho}}\left(\theta p_{4}\right)^{\mu}-\bar{\sigma}^{\mu \dot{\alpha} \alpha}\left(p_{2} \theta p_{4}\right)\right) f_{\star_{2}}\left(p_{1}, p_{3}\right) f_{\star_{2}}\left(p_{2}, p_{4}\right) \\
& +\frac{i}{2}\left(\bar{\sigma}^{\rho \dot{\alpha} \alpha} p_{2_{\rho}}\left(\left(\theta p_{3}\right)^{\mu}\left(\theta p_{4}\right)^{\nu}-\left(p_{3} \theta p_{4}\right) \theta^{\mu \nu}\right)-\bar{\sigma}^{\nu \dot{\alpha} \alpha}\left(\left(p_{2} \theta p_{3}\right)\left(\theta p_{4}\right)^{\mu}+\left(p_{2} \theta p_{4}\right)\left(\theta p_{3}\right)^{\mu}\right)\right) \\
& \cdot\left(f_{\star_{3^{\prime}}}\left(p_{2}, p_{4}, p_{3}\right)+f_{\star_{3^{\prime}}}\left(p_{4}, p_{2}, p_{3}\right)\right) \\
& +\frac{i}{2}\left(\bar{\sigma}^{\rho \dot{\alpha} \alpha} p_{3_{\rho}}\left(\left(\theta p_{4}\right)^{\mu}\left(\theta p_{2}\right)^{\nu}+\left(p_{2} \theta p_{4}\right) \theta^{\mu \nu}\right)+\bar{\sigma}^{\mu \dot{\alpha} \alpha}\left(\left(p_{2} \theta p_{3}\right)\left(\theta p_{4}\right)^{\nu}-\left(p_{3} \theta p_{4}\right)\left(\theta p_{2}\right)^{\nu}\right)\right) \\
& \cdot\left(f_{\star_{3^{\prime}}}\left(p_{3}, p_{4}, p_{2}\right)+f_{\star_{3^{\prime}}}\left(p_{4}, p_{3}, p_{2}\right)\right) \\
& +\frac{i}{2}\left(\theta p_{3}\right)^{\mu}\left(\bar{\sigma}^{\rho \dot{\alpha} \alpha} p_{3_{\rho}}\left(\theta p_{4}\right)^{\nu}-\bar{\sigma}^{\nu \dot{\alpha} \alpha}\left(p_{3} \theta p_{4}\right)\right) \\
& \cdot\left(f_{\star_{3^{\prime}}}\left(p_{4}, p_{2}, p_{3}\right)+f_{\star_{3^{\prime}}}\left(p_{2}, p_{3}, p_{4}\right)-2 f_{\star_{2}}\left(p_{1}, p_{2}\right) f_{\star_{2}}\left(p_{3}, p_{4}\right)\right) \\
& +\frac{i}{2}\left(\theta p_{4}\right)^{\mu}\left(\bar{\sigma}^{\rho \dot{\alpha} \alpha} p_{3_{\rho}}\left(\theta p_{4}\right)^{\nu}-\bar{\sigma}^{\nu \dot{\alpha} \alpha}\left(p_{3} \theta p_{4}\right)\right) \\
& \cdot\left(f_{\star_{3^{\prime}}}\left(p_{3}, p_{2}, p_{4}\right)+f_{\star_{3^{\prime}}}\left(p_{2}, p_{3}, p_{4}\right)-2 f_{\star_{2}}\left(p_{1}, p_{2}\right) f_{\star_{2}}\left(p_{3}, p_{4}\right)\right) \\
& +\frac{i}{2}\left(\theta p_{2}\right)^{\nu}\left(\bar{\sigma}^{\rho \dot{\alpha} \alpha} p_{2_{\rho}}\left(\theta p_{4}\right)^{\mu}-\bar{\sigma}^{\mu \dot{\alpha} \alpha}\left(p_{2} \theta p_{4}\right)\right) \\
& \cdot\left(f_{\star_{3^{\prime}}}\left(p_{4}, p_{3}, p_{2}\right)+f_{\star_{3^{\prime}}}\left(p_{3}, p_{2}, p_{4}\right)-2 f_{\star_{2}}\left(p_{1}, p_{3}\right) f_{\star_{2}}\left(p_{2}, p_{4}\right)\right) \\
& +\frac{i}{2}\left(\theta p_{4}\right)^{\nu}\left(\bar{\sigma}^{\rho \dot{\alpha} \alpha} p_{2 \rho}\left(\theta p_{4}\right)^{\mu}-\bar{\sigma}^{\mu \dot{\alpha} \alpha}\left(p_{2} \theta p_{4}\right)\right) \\
& \cdot\left(f_{\star_{3^{\prime}}}\left(p_{2}, p_{3}, p_{4}\right)+f_{\star_{3^{\prime}}}\left(p_{3}, p_{2}, p_{4}\right)-2 f_{\star_{2}}\left(p_{1}, p_{3}\right) f_{\star_{2}}\left(p_{2}, p_{4}\right)\right) \\
& +\frac{i}{2} \bar{\sigma}^{\rho \dot{\alpha} \alpha} p_{1_{\rho}}\left(\left(f_{\star_{2}}\left(p_{1}, p_{2}\right) f_{\star_{2}}\left(p_{3}, p_{4}\right)+f_{\star_{2}}\left(p_{1}, p_{3}\right) f_{\star_{2}}\left(p_{2}, p_{4}\right)\right)\left(\theta p_{2}\right)^{\mu}\left(\theta p_{3}\right)^{\nu}\right. \\
& -f_{\star_{3}^{\prime}}\left(p_{4}, p_{2}, p_{3}\right)\left(\left(p_{2} \theta p_{3}\right) \theta^{\mu \nu}+\left(\theta p_{3}\right)^{\mu}\left(\theta p_{2}\right)^{\nu}\right) \\
& -\left(\theta p_{2}\right)^{\mu}\left(\left(p_{2} \theta p_{3}\right)\left(\theta p_{4}\right)^{\nu}+\left(\theta p_{2}\right)^{\nu}\left(p_{3} \theta p_{4}\right)\right) f_{(\mathrm{I})}\left(p_{2}, p_{3}, p_{4}\right) \\
& +\left(\theta p_{3}\right)^{\nu}\left(\left(p_{2} \theta p_{3}\right)\left(\theta p_{4}\right)^{\mu}-\left(\theta p_{3}\right)^{\mu}\left(p_{2} \theta p_{4}\right)\right) f_{(\mathrm{I})}\left(p_{3}, p_{2}, p_{4}\right) \\
& \left.-\frac{1}{2}\left(\theta p_{2}\right)^{\mu}\left(\theta p_{3}\right)^{\nu}\left(p_{2} \theta p_{3}\right)\left(f_{(\mathrm{I})}\left(p_{2}, p_{3}, p_{4}\right)-f_{(\mathrm{I})}\left(p_{3}, p_{2}, p_{4}\right)\right)\right),
\end{aligned}
$$

with photon momenta $\left(p_{2}, \mu\right),\left(p_{3}, \nu\right)$ and photino momentum $p_{1}$ being incoming. 


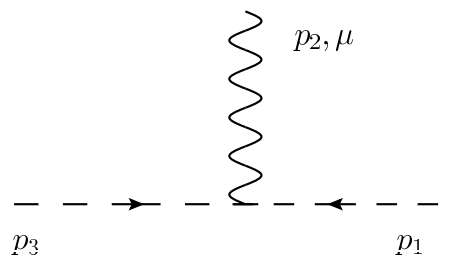

Figure 38. Scalar-photon vertex: $S^{\mu}\left(p_{1}, p_{2}, p_{3}\right) ; p_{1}+p_{2}+p_{3}=0$.

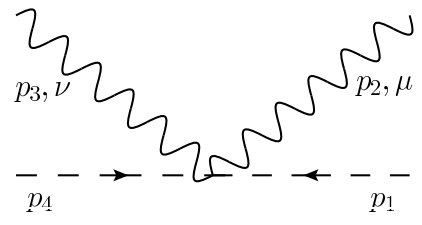

Figure 39. Scalar-2photons vertex: $S_{\text {rele }}^{\mu \nu}\left(p_{1}, p_{2}, p_{3}, p_{4}\right) ; p_{1}+p_{2}+p_{3}+p_{4}=0$.

\section{Scalar-photon Feynman rules}

From the scalar action (3.3) we obtain the following scalar-photon Feynman rule corresponding to the figure 38 :

$$
\begin{aligned}
S^{\mu}\left(p_{1}, p_{2}, p_{3}\right)= & -e f_{\star_{2}}\left(p_{1}, p_{3}\right)\left(\left(p_{1} p_{2}\right)\left(\theta p_{3}\right)^{\mu}-\left(p_{1} p_{3}\right)\left(\theta p_{2}\right)^{\mu}+\left(p_{2} p_{3}\right)\left(\theta p_{1}\right)^{\mu}\right. \\
& \left.-p_{1}^{\mu}\left(p_{2} \theta p_{3}\right)-p_{3}^{\mu}\left(p_{2} \theta p_{1}\right)\right)
\end{aligned}
$$

and the following scalar-2photons Feynman rule corresponding to the figure 39:

$$
\begin{aligned}
& S_{\text {rele }}^{\mu \nu}\left(p_{1}, p_{2}, p_{3}, p_{4}\right)=S_{1^{\prime}}^{\mu \nu}+S_{2^{\prime}}^{\mu \nu}+S_{3^{\prime}}^{\mu \nu}+S_{4^{\prime}}^{\mu \nu} \\
& S_{1^{\prime}}^{\mu \nu}=i e^{2}\left\{f _ { \star _ { 2 } } ( p _ { 1 } , p _ { 2 } ) f _ { \star _ { 2 } } ( p _ { 4 } , p _ { 3 } ) \cdot \left(\left(p_{2} p_{3}\right)\left(\theta p_{1}\right)^{\nu}\left(\theta p_{1}\right)^{\mu}-p_{2}^{\nu}\left(\theta p_{4}\right)^{\mu}\left(p_{3} \theta p_{4}\right)\right.\right. \\
& \left.-p_{3}^{\mu}\left(\theta p_{4}\right)^{\nu}\left(p_{2} \theta p_{1}\right)+g^{\mu \nu}\left(p_{3} \theta p_{4}\right)\left(p_{2} \theta p_{1}\right)\right)+f_{\star_{2}}\left(p_{1}, p_{3}\right) f_{\star_{2}}\left(p_{4}, p_{2}\right) \\
& \cdot\left(\left(p_{2} p_{3}\right)\left(\theta p_{1}\right)^{\nu}\left(\theta p_{4}\right)^{\mu}-p_{2}^{\nu}\left(\theta p_{4}\right)^{\mu}\left(p_{3} \theta p_{1}\right)-p_{3}^{\mu}\left(\theta p_{1}\right)^{\nu}\left(p_{2} \theta p_{4}\right)+g^{\mu \nu}\left(p_{2} \theta p_{4}\right)\left(p_{3} \theta p_{1}\right)\right) \\
& -\left(\left(p_{1} p_{3}\right)\left(\theta p_{2}\right)^{\nu}\left(\theta p_{4}\right)^{\mu}-p_{1}^{\nu}\left(\theta p_{2}\right)^{\mu}\left(p_{3} \theta p_{4}\right)\right. \\
& \left.-p_{4}^{\mu}\left(\theta p_{3}\right)^{\nu}\left(p_{2} \theta p_{1}\right)+\left(p_{4} p_{2}\right)\left(\theta p_{2}\right)^{\nu}\left(\theta p_{1}\right)^{\mu}\right)+f_{\star_{2}}\left(p_{2}, p_{4}\right) f_{\star_{2}}\left(p_{3}, p_{1}\right) \\
& \left.\cdot\left(\left(p_{4} p_{3}\right)\left(\theta p_{2}\right)^{\mu}\left(\theta p_{1}\right)^{\nu}-p_{4}^{\nu}\left(\theta p_{2}\right)^{\mu}\left(p_{3} \theta p_{1}\right)-p_{1}^{\mu}\left(\theta p_{3}\right)^{\nu}\left(p_{2} \theta p_{4}\right)+\left(p_{1} p_{2}\right)\left(\theta p_{3}\right)^{\nu}\left(\theta p_{4}\right)^{\mu}\right)\right\}
\end{aligned}
$$




$$
\begin{aligned}
& S_{2^{\prime}}^{\mu \nu}=\frac{i}{2} e^{2}\left\{[ f _ { \star _ { 3 ^ { \prime } } } ( p _ { 2 } , p _ { 3 } , p _ { 4 } ) + f _ { \star _ { 3 ^ { \prime } } } ( p _ { 4 } , p _ { 2 } , p _ { 3 } ) ] \cdot \left(\left(p_{1} p_{2}\right)\left(\theta p_{3}\right)^{\mu}\left(\theta p_{4}\right)^{\nu}-\left(p_{1} p_{2}\right) \theta^{\mu \nu}\left(p_{3} \theta p_{4}\right)\right.\right. \\
& \left.-p_{1}^{\mu}\left(\theta p_{4}\right)^{\nu}\left(p_{2} \theta p_{3}\right)-p_{1}^{\mu}\left(\theta p_{2}\right)^{\nu}\left(p_{3} \theta p_{4}\right)\right)+\left[f_{\star_{3^{\prime}}}\left(p_{2}, p_{3}, p_{1}\right)+f_{\star_{3^{\prime}}}\left(p_{1}, p_{2}, p_{3}\right)\right] \\
& \cdot\left(\left(p_{4} p_{2}\right)\left(\theta p_{3}\right)^{\mu}\left(\theta p_{1}\right)^{\nu}-\left(p_{4} p_{2}\right)\left(p_{3} \theta p_{1}\right) \theta^{\mu \nu}-p_{4}^{\mu}\left(\theta p_{1}\right)^{\nu}\left(p_{2} \theta p_{3}\right)-p_{4}^{\mu}\left(\theta p_{2}\right)^{\nu}\left(p_{3} \theta p_{1}\right)\right) \\
& +\left[f_{\star_{3^{\prime}}}\left(p_{3}, p_{2}, p_{4}\right)+f_{\star_{3^{\prime}}}\left(p_{4}, p_{3}, p_{2}\right)\right] \cdot\left(\left(p_{1} p_{3}\right)\left(\theta p_{2}\right)^{\nu}\left(\theta p_{4}\right)^{\mu}-\left(p_{1} p_{3}\right)\left(p_{2} \theta p_{3}\right) \theta^{\mu \nu}\right. \\
& \left.-p_{1}^{\nu}\left(\theta p_{3}\right)^{\mu}\left(p_{3} \theta p_{2}\right)-p_{1}^{\nu}\left(\theta p_{3}\right)^{\mu}\left(p_{2} \theta p_{4}\right)\right)+\left[f_{\star_{3^{\prime}}}\left(p_{3}, p_{2}, p_{1}\right)+f_{\star_{3^{\prime}}}\left(p_{1}, p_{3}, p_{2}\right)\right] \\
& \left.\cdot\left(\left(p_{4} p_{3}\right)\left(\theta p_{2}\right)^{\nu}\left(\theta p_{1}\right)^{\mu}-\left(p_{4} p_{3}\right)\left(p_{2} \theta p_{1}\right) \theta^{\mu \nu}-p_{4}^{\nu}\left(\theta p_{1}\right)^{\mu}\left(p_{3} \theta p_{2}\right)-p_{4}^{\nu}\left(\theta p_{3}\right)^{\mu}\left(p_{2} \theta p_{1}\right)\right)\right\} \text {, } \\
& S_{3^{\prime}}^{\mu \nu}=\frac{i}{2} e^{2}\left\{\left[f_{\star_{3^{\prime}}}\left(p_{4}, p_{2}, p_{3}\right)+f_{\star_{3^{\prime}}}\left(p_{2}, p_{3}, p_{4}\right)-2 f_{\star_{2}}\left(p_{2}, p_{1}\right) f_{\star_{2}}\left(p_{3}, p_{4}\right)\right]\right. \\
& \cdot\left(\left(p_{1} p_{3}\right)\left(\theta p_{3}\right)^{\mu}\left(\theta p_{4}\right)^{\nu}-p_{1}^{\nu}\left(\theta p_{3}\right)^{\mu}\left(p_{3} \theta p_{4}\right)\right)+\left[f_{\star_{3^{\prime}}}\left(p_{1}, p_{2}, p_{3}\right)+f_{\star_{3^{\prime}}}\left(p_{2}, p_{3}, p_{1}\right)\right. \\
& \left.-2 f_{\star_{2}}\left(p_{2}, p_{4}\right) f_{\star_{2}}\left(p_{3}, p_{1}\right)\right] \cdot\left(\left(p_{4} p_{3}\right)\left(\theta p_{3}\right)^{\mu}\left(\theta p_{1}\right)^{\nu}-p_{4}^{\nu}\left(\theta p_{3}\right)^{\mu}\left(p_{3} \theta p_{1}\right)\right) \\
& +\left[f_{\star_{3^{\prime}}}\left(p_{4}, p_{3}, p_{2}\right)+f_{\star_{3^{\prime}}}\left(p_{3}, p_{2}, p_{4}\right)-2 f_{\star_{2}}\left(p_{3}, p_{1}\right) f_{\star_{2}}\left(p_{2}, p_{4}\right)\right] \\
& \cdot\left(\left(p_{1} p_{2}\right)\left(\theta p_{2}\right)^{\nu}\left(\theta p_{4}\right)^{\mu}-p_{1}^{\mu}\left(\theta p_{2}\right)^{\nu}\left(p_{2} \theta p_{4}\right)\right)+\left[f_{\star_{3^{\prime}}}\left(p_{1}, p_{3}, p_{2}\right)+f_{\star_{3^{\prime}}}\left(p_{3}, p_{2}, p_{1}\right)\right. \\
& \left.-2 f_{\star_{2}}\left(p_{3}, p_{4}\right) f_{\star_{2}}\left(p_{2}, p_{1}\right)\right] \cdot\left(\left(p_{4} p_{2}\right)\left(\theta p_{2}\right)^{\nu}\left(\theta p_{1}\right)^{\mu}-p_{4}^{\mu}\left(\theta p_{2}\right)^{\nu}\left(p_{2} \theta p_{1}\right)\right) \\
& +\left[f_{\star_{3^{\prime}}}\left(p_{2}, p_{3}, p_{4}\right)+f_{\star_{3^{\prime}}}\left(p_{3}, p_{2}, p_{4}\right)-2 f_{\star_{2}}\left(p_{2}, p_{1}\right) f_{\star_{2}}\left(p_{3}, p_{4}\right)\right] \\
& \cdot\left(\left(p_{1} p_{3}\right)\left(\theta p_{4}\right)^{\mu}\left(\theta p_{4}\right)^{\nu}-p_{1}^{\nu}\left(\theta p_{4}\right)^{\mu}\left(p_{3} \theta p_{4}\right)\right)+\left[f_{\star_{3^{\prime}}}\left(p_{2}, p_{3}, p_{1}\right)+f_{\star_{3^{\prime}}}\left(p_{3}, p_{2}, p_{1}\right)\right. \\
& \left.-2 f_{\star_{2}}\left(p_{2}, p_{4}\right) f_{\star_{2}}\left(p_{3}, p_{1}\right)\right] \cdot\left(\left(p_{4} p_{3}\right)\left(\theta p_{1}\right)^{\mu}\left(\theta p_{1}\right)^{\nu}-p_{4}^{\nu}\left(\theta p_{1}\right)^{\mu}\left(p_{3} \theta p_{1}\right)\right) \\
& +\left[f_{\star_{3^{\prime}}}\left(p_{3}, p_{2}, p_{4}\right)+f_{\star_{3^{\prime}}}\left(p_{2}, p_{3}, p_{4}\right)-2 f_{\star^{\prime} 2}\left(p_{3}, p_{1}\right) f_{\star_{2}}\left(p_{2}, p_{4}\right)\right] \\
& \cdot\left(\left(p_{1} p_{2}\right)\left(\theta p_{4}\right)^{\nu}\left(\theta p_{4}\right)^{\mu}-p_{1}^{\mu}\left(\theta p_{4}\right)^{\nu}\left(p_{2} \theta p_{4}\right)\right)+\left[f_{\star_{3^{\prime}}}\left(p_{3}, p_{2}, p_{1}\right)+f_{\star_{3^{\prime}}}\left(p_{2}, p_{3}, p_{1}\right)\right. \\
& \left.\left.-2 f_{\star_{2}}\left(p_{3}, p_{4}\right) f_{\star_{2}}\left(p_{2}, p_{1}\right)\right] \cdot\left(\left(p_{4} p_{2}\right)\left(\theta p_{1}\right)^{\nu}\left(\theta p_{1}\right)^{\mu}-p_{4}^{\mu}\left(\theta p_{1}\right)^{\nu}\left(p_{2} \theta p_{1}\right)\right)\right\}, \\
& S_{4^{\prime}}^{\mu \nu}=\frac{i}{2} e^{2}\left(p_{1} \cdot p_{4}\right)\left(\left(f_{\star_{2}}\left(p_{1}, p_{2}\right) f_{\star_{2}}\left(p_{3}, p_{4}\right)+f_{\star_{2}}\left(p_{1}, p_{3}\right) f_{\star_{2}}\left(p_{2}, p_{4}\right)\right)\left(\theta p_{2}\right)^{\mu}\left(\theta p_{3}\right)^{\nu}\right. \\
& -f_{\star_{3}^{\prime}}\left(p_{4}, p_{2}, p_{3}\right)\left(\left(p_{2} \theta p_{3}\right) \theta^{\mu \nu}+\left(\theta p_{3}\right)^{\mu}\left(\theta p_{2}\right)^{\nu}\right) \\
& -\left(\theta p_{2}\right)^{\mu}\left(\left(p_{2} \theta p_{3}\right)\left(\theta p_{4}\right)^{\nu}+\left(\theta p_{2}\right)^{\nu}\left(p_{3} \theta p_{4}\right)\right) f_{(\mathrm{I})}\left(p_{2}, p_{3}, p_{4}\right) \\
& +\left(\theta p_{3}\right)^{\nu}\left(\left(p_{2} \theta p_{3}\right)\left(\theta p_{4}\right)^{\mu}-\left(\theta p_{3}\right)^{\mu}\left(p_{2} \theta p_{4}\right)\right) f_{(\mathrm{I})}\left(p_{3}, p_{2}, p_{4}\right) \\
& \left.-\frac{1}{2}\left(\theta p_{2}\right)^{\mu}\left(\theta p_{3}\right)^{\nu}\left(p_{2} \theta p_{3}\right)\left(f_{(\mathrm{I})}\left(p_{2}, p_{3}, p_{4}\right)-f_{(\mathrm{I})}\left(p_{3}, p_{2}, p_{4}\right)\right)\right) .
\end{aligned}
$$




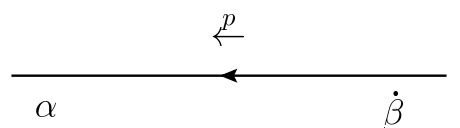

Figure 40. $\mathcal{N}=2$ fermion propagator: $\Sigma_{\alpha \dot{\beta}}(p)$.

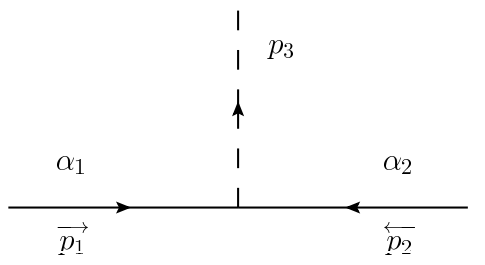

Figure 41. $\mathcal{N}=2$ scalar-fermion vertex: $\Gamma^{\alpha_{1} \alpha_{2}}\left(p_{1}, p_{2}\right) ; p_{1}+p_{2}-p_{3}=0$.

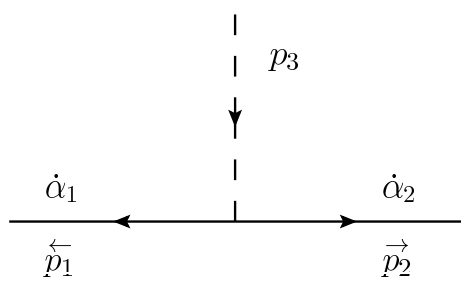

Figure 42. $\mathcal{N}=2$ scalar-antifermion vertex: $\Gamma^{\dot{\alpha}_{1} \dot{\alpha}_{2}}\left(p_{1}, p_{2}\right) ; p_{3}-p_{1}-p_{2}=0$.

\section{E Scalar-fermion Feynman rules in the $\mathrm{NC} \mathcal{N}=2,4 \mathrm{SYM} \mathrm{U}(1)$}

Feynman rules in $\mathcal{N}=2$ from figures $40-43$ are:

$$
\begin{aligned}
\Sigma_{\alpha \dot{\beta}}(p) & =i \frac{\sigma_{\alpha \dot{\beta}}^{\mu} p_{\mu}}{p^{2}}, \\
\Gamma^{\alpha_{1} \alpha_{2}}\left(p_{1}, p_{2}\right) & =-2 \sqrt{2} i e \sin \frac{p_{1} \theta p_{2}}{2} \epsilon^{\alpha_{1} \alpha_{2}}, \\
\Gamma^{\dot{\alpha}_{1} \dot{\alpha}_{2}}\left(p_{1}, p_{2}\right) & =2 \sqrt{2} i e \sin \frac{p_{1} \theta p_{2}}{2} \epsilon^{\dot{\alpha}_{1} \dot{\alpha}_{2}}, \\
\Gamma\left(p_{1}, p_{2}, p_{3}, p_{4}\right) & =4 i e^{2}\left[\sin \frac{p_{1} \theta p_{4}}{2} \sin \frac{p_{2} \theta p_{3}}{2}+\{1 \leftrightarrow 2\}\right] .
\end{aligned}
$$

Feynman rules in $\mathcal{N}=4$ from figures $44-47$ are:

$$
\begin{aligned}
\Sigma_{i}^{j}(p) & =i \frac{\sigma^{\mu} p_{\mu} \delta_{i}^{j}}{p^{2}} \\
\Gamma_{i_{1} i_{2} m}^{\alpha_{1} \alpha_{2}}\left(p_{1}, p_{2}\right) & =2 i e\left(\tilde{\sigma}_{m}^{-1}\right)_{i_{1} i_{2}} \sin \frac{p_{1} \theta p_{2}}{2} \epsilon^{\alpha_{1} \alpha_{2}} \\
\Gamma_{i_{1} i_{2} m}^{\dot{\alpha}_{1} \dot{\alpha}_{2}}\left(p_{1}, p_{2}\right) & =2 i e\left(\tilde{\sigma}_{m}\right)_{i_{1} i_{2}} \sin \frac{p_{1} \theta p_{2}}{2} \epsilon^{\dot{\alpha}_{1} \dot{\alpha}_{2}} \\
\Gamma_{m_{1} m_{2} m_{3} m_{4}}\left(p_{1}, p_{2}, p_{3}, p_{4}\right) & =-4 i e^{2}\left[\sin \frac{p_{1} \theta p_{2}}{2} \sin \frac{p_{3} \theta p_{4}}{2}\left(\delta_{m_{1} m_{3}} \delta_{m_{2} m_{4}}-\delta_{m_{2} m_{3}} \delta_{m_{1} m_{4}}\right)+c \cdot p \cdot\right] .
\end{aligned}
$$




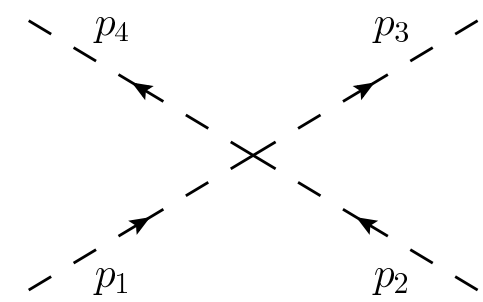

Figure 43. $\mathcal{N}=2$ four-scalar vertex: $\Gamma\left(p_{1}, p_{2}, p_{3}, p_{4}\right) ; p_{1}+p_{2}-p_{3}-p_{4}=0$.

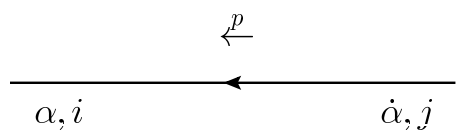

Figure 44. $\mathcal{N}=4$ fermion propagator: $\Sigma_{i}^{j}(p)$.

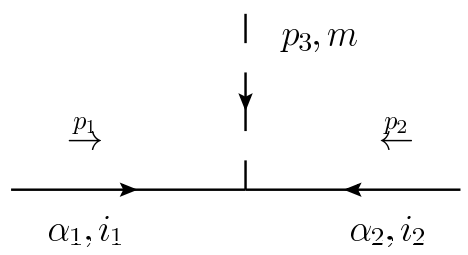

Figure 45. $\mathcal{N}=4$ scalar-fermion vertex: $\Gamma_{i_{1} i_{2} m}^{\alpha_{1} \alpha_{2}}\left(p_{1}, p_{2}\right) ; p_{1}+p_{2}+p_{3}=0$.

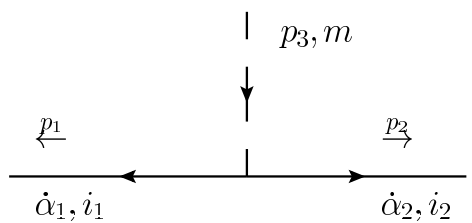

Figure 46. $\mathcal{N}=4$ scalar-antifermion vertex: $\Gamma_{i_{1} i_{2} m}^{\dot{\alpha}_{1} \dot{\alpha}_{2}}\left(p_{1}, p_{2}\right) ; p_{3}-p_{1}-p_{2}=0$.

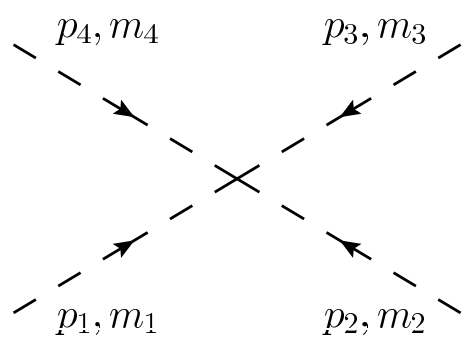

Figure 47. $\mathcal{N}=4$ four-scalar vertex: $\Gamma_{m_{1} m_{2} m_{3} m_{4}}\left(p_{1}, p_{2}, p_{3}, p_{4}\right) ; p_{1}+p_{2}+p_{3}+p_{4}=0$. 


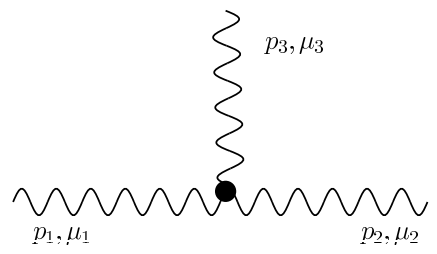

Figure 48. Gauge fixing 3-photon vertex: $\Gamma_{\mathrm{gf}}^{\mu_{1} \mu_{2} \mu_{3}}\left(p_{1}, p_{2}, p_{3}\right) ; p_{1}+p_{2}+p_{3}=0$.

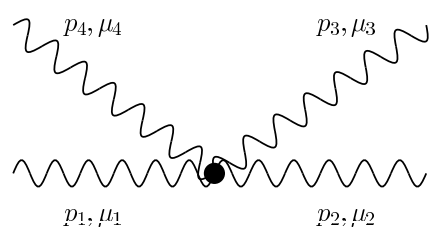

Figure 49. Gauge fixing 4-photon vertex: $\Gamma_{\mathrm{gf}}^{\mu_{1} \mu_{2} \mu_{3} \mu_{4}}\left(p_{1}, p_{2}, p_{3}, p_{4}\right) ; p_{1}+p_{2}+p_{3}+p_{4}=0$.

\section{F Feynman rules from the NC gauge fixing and ghost actions}

From figures 48-51 we have the following Feynman rules:

$$
\begin{aligned}
& \Gamma_{\mathrm{gf}}^{\mu_{1} \mu_{2} \mu_{3}}\left(p_{1}, p_{2}, p_{3}\right)=-\frac{1}{2} f_{\star_{2}}\left(p_{1}, p_{2}\right)\left[2 p_{1}^{\mu_{1}} p_{1}^{\mu_{3}}\left(\theta p_{3}\right)^{\mu_{2}}-p_{1}^{\mu_{1}}\left(p_{1} p_{3}\right) \theta^{\mu_{2} \mu_{3}}+2 p_{1}^{\mu_{1}} p_{1}^{\mu_{2}}\left(\theta p_{2}\right)^{\mu_{3}}\right. \\
& -p_{1}^{\mu_{1}}\left(p_{1} p_{2}\right) \theta^{\mu_{2} \mu_{3}}+2 p_{2}^{\mu_{2}} p_{2}^{\mu_{3}}\left(\theta p_{3}\right)^{\mu_{1}}-p_{2}^{\mu_{2}}\left(p_{2} p_{3}\right) \theta^{\mu_{1} \mu_{3}}+2 p_{2}^{\mu_{2}} p_{2}^{\mu_{1}}\left(\theta p_{1}\right)^{\mu_{3}}-p_{2}^{\mu_{2}}\left(p_{2} p_{1}\right) \theta^{\mu_{1} \mu_{3}} \\
& +2 p_{3}^{\mu_{3}} p_{3}^{\mu_{2}}\left(\theta p_{2}\right)^{\mu_{1}}-p_{3}^{\mu_{3}}\left(p_{3} p_{2}\right) \theta^{\mu_{1} \mu_{2}}+\left(p_{3}^{\mu_{3}} p_{3}^{\mu_{1}}\left(\theta p_{1}\right)^{\mu_{2}}-p_{3}^{\mu_{1}}\left(p_{1} p_{3}\right) \theta^{\mu_{1} \mu_{2}}\right], \\
& \Gamma_{\mathrm{gf}}^{\mu_{1} \mu_{2} \mu_{3} \mu_{4}}\left(p_{1}, p_{2}, p_{3}, p_{4}\right)=-\frac{i}{8} f_{\star_{2}}\left(p_{1}, p_{2}\right) f_{\star_{2}}\left(p_{3}, p_{4}\right)\left[\left(p_{1}+p_{2}\right)^{\mu_{2}}\left(\theta p_{2}\right)^{\mu_{1}}-\left(p_{1}+p_{2}\right) \cdot p_{1} \theta^{\mu_{1} \mu_{2}}\right] \\
& \cdot\left[\left(p_{3}+p_{4}\right)^{\mu_{4}}\left(\theta p_{4}\right)^{\mu_{3}}-\left(p_{1}+p_{4}\right) \cdot p_{4} \theta^{\mu_{3} \mu_{4}}\right] \\
& +\frac{i}{8} f_{\star_{3^{\prime}}}\left[p_{2}, p_{3}, p_{4}\right]\left[2 p_{1}^{\mu_{1}} p_{1}^{\mu_{4}}\left(2\left(\theta p_{4}\right)^{\mu_{2}}\left(\theta p_{4}\right)^{\mu_{3}}-\left(p_{3} \theta p_{4}\right) \theta^{\mu_{2} \mu_{3}}\right)\right. \\
& +2 p_{1}^{\mu_{1}} p_{1}^{\mu_{2}}\left(2\left(\theta p_{2}\right)^{\mu_{4}}\left(\theta p_{4}\right)^{\mu_{3}}+\left(p_{2} \theta p_{4}\right) \theta^{\mu_{3} \mu_{4}}\right)+p_{1}^{\mu_{1}}\left(p_{1} p_{2}\right)\left(2\left(\theta p_{4}\right)^{\mu_{3}} \theta^{\mu_{2} \mu_{4}}-\left(\theta p_{4}\right)^{\mu_{2}} \theta^{\mu_{3} \mu_{4}}\right) \\
& \left.-p_{1}^{\mu_{1}}\left(p_{1} p_{4}\right)\left(3\left(\theta p_{3}\right)^{\mu_{2}} \theta^{\mu_{3} \mu_{4}}+2\left(\theta p_{3}\right)^{\mu_{4}} \theta^{\mu_{2} \mu_{3}}+2\left(\theta p_{4}\right)^{\mu_{3}} \theta^{\mu_{2} \mu_{4}}+\left(\theta p_{4}\right)^{\mu_{2}} \theta^{\mu_{3} \mu_{4}}\right)\right] \\
& +\left\{S_{4} \text { permutations }\right\} \text {, } \\
& \Gamma_{\text {gh }}^{\mu}\left(p_{1}, p_{2}\right)=f_{\star_{2}}\left(p_{1}, p_{2}\right)\left[\frac{1}{2}\left(p_{1}+p_{2}\right)^{2}\left(\theta p_{2}\right)^{\mu}-\left(p_{1}+p_{2}\right)^{\mu}\left(p_{1} \theta p_{2}\right)\right], \\
& \Gamma_{\mathrm{gh}}^{\mu_{1} \mu_{2}}\left(p_{1}, p_{2}, p_{3}, p_{4}\right)=\left\{\frac{1}{2} f_{\star_{2}}\left(p_{1}, p_{2}\right) f_{\star_{2}}\left(p_{3}, p_{4}\right)\left[2 p_{4}^{\mu_{2}}\left(\theta p_{2}\right)^{\mu_{1}}-\left(p_{4} p_{1}\right) \theta^{\mu_{1} \mu_{2}}\right]\left(p_{4} \theta p_{3}\right)\right. \\
& \left.+\frac{1}{2} f_{\star_{2}}\left(p_{2}, p_{3}\right) f_{\star_{2}}\left(p_{1}, p_{4}\right) p_{4}^{\mu_{1}}\left(\theta p_{3}\right)^{\mu_{2}}\left(p_{1} \theta p_{4}\right)\right\}+\{1 \leftrightarrow 2\} .
\end{aligned}
$$




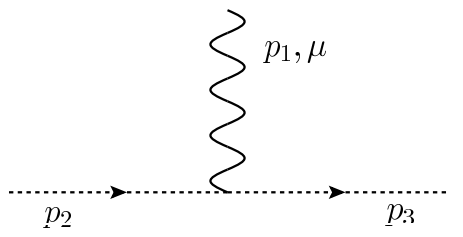

Figure 50. Ghost-photon vertex: $\Gamma_{\mathrm{gh}}^{\mu}\left(p_{1}, p_{2}\right) ; p_{1}+p_{2}-p_{3}=0$.

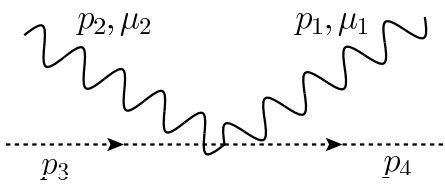

Figure 51. Ghost-2photons vertex: $\Gamma_{\mathrm{gh}}^{\mu_{1} \mu_{2}}\left(p_{1}, p_{2}, p_{3}, p_{4}\right) ; p_{1}+p_{2}+p_{3}-p_{4}=0$.

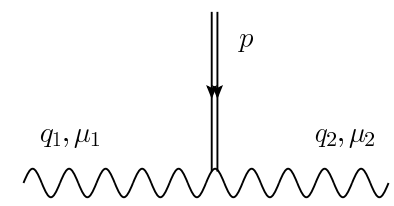

Figure 52. The 2gauge-Bauxiliary field interactions: $\Gamma_{B a a}^{\mu_{1} \mu_{2}}\left(p, q_{1}, q_{2}\right) ; p+q_{1}+q_{2}=0$.

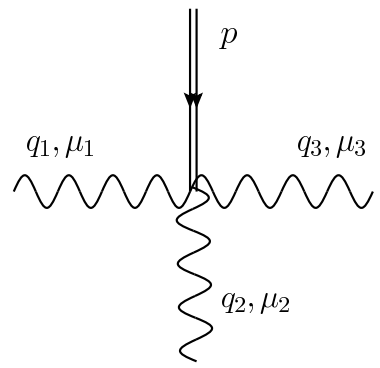

Figure 53. The 3gauge-Bauxiliary field interactions: $\Gamma_{B a a a}^{\mu_{1} \mu_{2} \mu_{3}}\left(p, q_{1}, q_{2}, q_{3}\right) ; p+q_{1}+q_{2}+q_{3}=0$.

\section{G Feynman rules from the gauge and BRST-auxiliary field interactions}

Feynman rule corresponding to figure 52 is:

$$
\Gamma_{\text {Baa }}^{\mu_{1} \mu_{2}}\left(p, q_{1}, q_{2}\right)=-\frac{i}{2} f_{\star_{2}}\left(q_{1}, q_{2}\right)\left(2 p^{\mu_{2}}\left(\theta q_{2}\right)^{\mu_{1}}-\left(p q_{2}\right) \theta^{\mu_{1} \mu_{2}}+2 p^{\mu_{1}}\left(\theta q_{1}\right)^{\mu_{2}}+\left(p q_{1}\right) \theta^{\mu_{1} \mu_{2}}\right),
$$

while the 3-gauge-B-auxiliary-field Feynman rule from figure 53 has the following form:

$$
\begin{aligned}
\Gamma_{\text {Baaa }}^{\mu_{1} \mu_{2} \mu_{3}}( & \left.p, q_{1}, q_{2}, q_{3}\right)=\frac{1}{8} f_{\star_{3^{\prime}}}\left(q_{1}, q_{2}, q_{3}\right)\left(3\left(p q_{3}\right)\left(\theta q_{2}\right)^{\mu_{1}} \theta^{\mu_{2} \mu_{3}}+\left(p q_{1}\right)\left(\theta q_{3}\right)^{\mu_{1}} \theta^{\mu_{2} \mu_{3}}\right. \\
& +\left(p q_{3}\right)\left(\theta q_{1}\right)^{\mu_{1}} \theta^{\mu_{2} \mu_{3}}-2\left(p q_{1}\right)\left(\theta q_{2}\right)^{\mu_{2}} \theta^{\mu_{1} \mu_{3}}+2\left(p q_{3}\right)\left(\theta q_{2}\right)^{\mu_{3}} \theta^{\mu_{1} \mu_{2}} \\
& +2\left(p q_{3}\right)\left(\theta q_{2}\right)^{\mu_{2}} \theta^{\mu_{1} \mu_{3}}+2 p^{\mu_{3}}\left(q_{2} \theta q_{3}\right) \theta^{\mu_{1} \mu_{2}}-2 p^{\mu_{1}}\left(q_{1} \theta q_{2}\right) \theta^{\mu_{2} \mu_{3}} \\
& \left.-4 p^{\mu_{3}}\left(\theta q_{2}\right)^{\mu_{1}}\left(\theta q_{3}\right)^{\mu_{2}}-4 p^{\mu_{1}}\left(\theta q_{3}\right)^{\mu_{2}}\left(\theta q_{1}\right)^{\mu_{3}}\right)+\left\{S_{3} \text { permutations }\right\} .
\end{aligned}
$$


Open Access. This article is distributed under the terms of the Creative Commons Attribution License (CC-BY 4.0), which permits any use, distribution and reproduction in any medium, provided the original author(s) and source are credited.

\section{References}

[1] D. Zanon, Noncommutative $N=1, N=2$ super $\mathrm{U}(N)$ Yang-Mills: UV/IR mixing and effective action results at one loop, Phys. Lett. B 502 (2001) 265 [hep-th/0012009] [INSPIRE].

[2] F.R. Ruiz, Gauge fixing independence of IR divergences in noncommutative U(1), perturbative tachyonic instabilities and supersymmetry, Phys. Lett. B 502 (2001) 274 [hep-th/0012171] [INSPIRE].

[3] A.F. Ferrari et al., Superfield covariant analysis of the divergence structure of noncommutative supersymmetric QED(4), Phys. Rev. D 69 (2004) 025008 [hep-th/0309154] [INSPIRE].

[4] L. Álvarez-Gaumé and M.A. Vazquez-Mozo, General properties of noncommutative field theories, Nucl. Phys. B 668 (2003) 293 [hep-th/0305093] [INSPIRE].

[5] A.F. Ferrari et al., Towards a consistent noncommutative supersymmetric Yang-Mills theory: Superfield covariant analysis, Phys. Rev. D 70 (2004) 085012 [hep-th/0407040] [INSPIRE].

[6] I. Jack and D.R.T. Jones, Ultraviolet finiteness in noncommutative supersymmetric theories, New J. Phys. 3 (2001) 19 [hep-th/0109195] [InSPIRE].

[7] A. Santambrogio and D. Zanon, One loop four point function in noncommutative $N=4$ Yang-Mills theory, JHEP 01 (2001) 024 [hep-th/0010275] [INSPIRE].

[8] M. Pernici, A. Santambrogio and D. Zanon, The One loop effective action of noncommutative $N=4$ super Yang-Mills is gauge invariant, Phys. Lett. B 504 (2001) 131 [hep-th/0011140] [INSPIRE].

[9] I.L. Buchbinder and I.B. Samsonov, Noncommutative $N=2$ supersymmetric theories in harmonic superspace, Grav. Cosmol. 8 (2002) 17 [hep-th/0109130] [INSPIRE].

[10] M. Hanada and H. Shimada, On the continuity of the commutative limit of the $4 d N=4$ non-commutative super Yang-Mills theory, Nucl. Phys. B 892 (2015) 449 [arXiv: 1410.4503] [INSPIRE].

[11] N. Seiberg and E. Witten, String theory and noncommutative geometry, JHEP 09 (1999) 032 [hep-th/9908142] [INSPIRE].

[12] P. Schupp and J. You, UV/IR mixing in noncommutative QED defined by Seiberg-Witten map, JHEP 08 (2008) 107 [arXiv:0807.4886] [INSPIRE].

[13] T. Mehen and M.B. Wise, Generalized *-products, Wilson lines and the solution of the Seiberg-Witten equations, JHEP 12 (2000) 008 [hep-th/0010204] [INSPIRE].

[14] B. Jurčo, L. Möller, S. Schraml, P. Schupp and J. Wess, Construction of nonAbelian gauge theories on noncommutative spaces, Eur. Phys. J. C 21 (2001) 383 [hep-th/0104153] [INSPIRE].

[15] J. Trampetic and J. You, $\theta$-exact Seiberg-Witten maps at order $e^{3}$, Phys. Rev. D 91 (2015) 125027 [arXiv:1501.00276] [InSPIRE]. 
[16] R. Horvat, A. Ilakovac, J. Trampetic and J. You, Self-energies on deformed spacetimes, JHEP 11 (2013) 071 [arXiv: 1306.1239] [INSPIRE].

[17] J. Trampetic and J. You, Two-Point Functions on Deformed Spacetime, SIGMA 10 (2014) 054 [arXiv: 1402.6184] [INSPIRE].

[18] R. Horvat, J. Trampetic and J. You, Photon self-interaction on deformed spacetime, Phys. Rev. D 92 (2015) 125006 [arXiv:1510.08691] [INSPIRE].

[19] C.P. Martin and C. Tamarit, The Seiberg-Witten map and supersymmetry, JHEP 11 (2008) 087 [arXiv: 0809.2684] [InSPIRE].

[20] H.K. Dreiner, H.E. Haber and S.P. Martin, Two-component spinor techniques and Feynman rules for quantum field theory and supersymmetry, Phys. Rept. 494 (2010) 1 [arXiv: 0812.1594] [INSPIRE].

[21] J.C. Collins, Renormalization. An Introduction To Renormalization, The Renormalization Group, And The Operator Product Expansion, Cambridge University Press, Cambridge U.K. (1984).

[22] M.F. Sohnius, Introducing Supersymmetry, Phys. Rept. 128 (1985) 39 [INSPIRE].

[23] G. Leibbrandt, Introduction to the Technique of Dimensional Regularization, Rev. Mod. Phys. 47 (1975) 849 [INSPIRE].

[24] S. Weinberg, High-energy behavior in quantum field theory, Phys. Rev. 118 (1960) 838 [INSPIRE].

[25] G. Leibbrandt, Introduction to Noncovariant Gauges, Rev. Mod. Phys. 59 (1987) 1067 [INSPIRE].

[26] G. Leibbrandt, C.P. Martin and M. Staley, Nonlocalities in field theory, Nucl. Phys. B 405 (1993) 777 [inSPIRE].

[27] G. Barnich, F. Brandt and M. Grigoriev, Local BRST cohomology and Seiberg-Witten maps in noncommutative Yang-Mills theory, Nucl. Phys. B 677 (2004) 503 [hep-th/0308092] [INSPIRE].

[28] Wolfram Research Inc., Mathematica, Version 8.0, Champaign U.S.A. (2010).

[29] J. Martin-Garcia, xAct, http://www.xact.es/. 